\title{
GUIDELINES FOR FINDING DATA CONCURRENCY PROBLEMS IN BUSINESS PROCESS MODELS
}

\author{
by \\ Kevin Burr \\ A thesis submitted to the Faculty of Graduate Studies and Research \\ in partial fulfillment of the requirements for the degree of \\ Master of Applied Science in Technology Innovation Management \\ Department of Systems and Computer Engineering \\ Carleton University \\ Ottawa, Canada, K1S 5B6
}

May, 2011

C Copyright 2011 Kevin Burr 


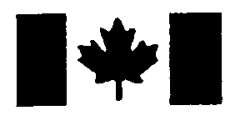

\author{
Library and Archives \\ Canada \\ Published Heritage \\ Branch \\ 395 Wellington Street \\ Ottawa ON K1A ON4 \\ Canada
}

Bibliotheque et

Archives Canada

Direction du

Patrimoine de l'édition

395 , rue Wellington

Ottawa ON K1A ON4

Canada
Your file Votre référence
ISBN: $978-0-494-81674-5$
Our file Notre référence
ISBN: $978-0-494-81674-5$
NOTICE:

The author has granted a nonexclusive license allowing Library and Archives Canada to reproduce, publish, archive, preserve, conserve, communicate to the public by telecommunication or on the Internet, loan, distribute and sell theses worldwide, for commercial or noncommercial purposes, in microform, paper, electronic and/or any other formats.

The author retains copyright ownership and moral rights in this thesis. Neither the thesis nor substantial extracts from it may be printed or otherwise reproduced without the author's permission.
AVIS:

L'auteur a accordé une licence non exclusive permettant à la Bibliothèque et Archives Canada de reproduire, publier, archiver, sauvegarder, conserver, transmettre au public par télécommunication ou par l'Internet, prêter, distribuer et vendre des thèses partout dans le monde, à des fins commerciales ou autres, sur support microforme, papier, électronique et/ou autres formats.

L'auteur conserve la propriété du droit d'auteur et des droits moraux qui protège cette thèse. $\mathrm{Ni}$ la thèse ni des extraits substantiels de celle-ci ne doivent être imprimés ou autrement reproduits sans son autorisation.
In compliance with the Canadian Privacy Act some supporting forms may have been removed from this thesis.

While these forms may be included in the document page count, their removal does not represent any loss of content from the thesis.
Conformément à la loi canadienne sur la protection de la vie privée, quelques formulaires secondaires ont été enlevés de cette thèse.

Bien que ces formulaires aient inclus dans la pagination, il n'y aura aucun contenu manquant. 
The undersigned hereby recommend to

the Faculty of Graduate Studies and Research acceptance of the thesis

\title{
GUIDELINES FOR FINDING DATA CONCURRENCY PROBLEMS IN BUSINESS PROCESS MODELS
}

\author{
Submitted by \\ Kevin Burr \\ In partial fulfillment of the requirements for the degree of \\ Master of Applied Science in Technology Innovation Management
}

Dr. Howard Schwartz, Department Chair

Dr. Michael Weiss, Thesis Supervisor

Carleton University

May, 2011 


\section{ABSTRACT}

Finding concurrency problems in software can be very hard because the problems may not appear until all of the components have been integrated together and just the right conditions occur. In production, these problems may even seem to be random. In software, these problems have been well researched, however research in Business Process Modeling is still relatively new, and the people who are experts on business processes may not be familiar with all the knowledge contained within the realm of software engineering.

This thesis adapts well known solutions to common data concurrency problems from software engineering, to guidelines business process modelers can use to detect where potential problems may hide and suggest possible solutions. In software engineering terms, the thesis takes the literature on data concurrency patterns and principles and combines it to form a catalog of process model smells (like symptoms) with references back to how the problems should be dealt with.

In order to create these guidelines, a pattern analysis framework is described which amalgamates existing pattern research and re-categorizes it by smells instead of solutions as patterns are typically organized. It also synthesizes new information by revealing new relationships between different research sources. 


\title{
ACKNOWLEDGMENTS
}

\author{
I would like to thank God, \\ without Whose help this thesis would not have been possible, \\ Jenny, Tara and Kacie for their patience, \\ and Professors Weiss and King for their guidance.
}




\section{TABLE OF CONTENTS}

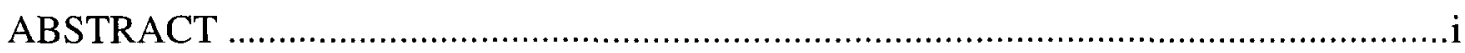

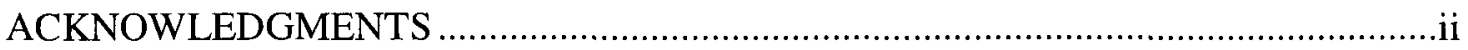

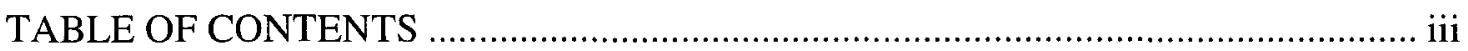

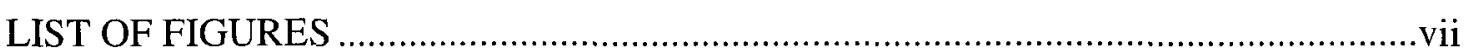

LIST OF TABLES .............................................................................................. vii

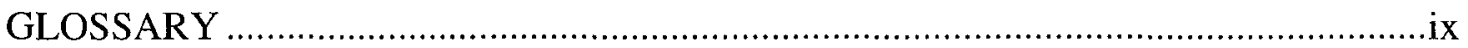

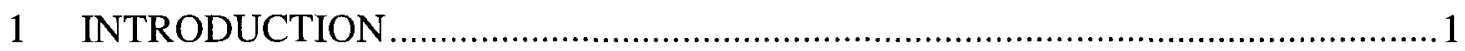

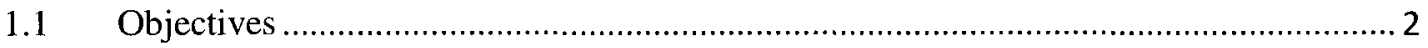

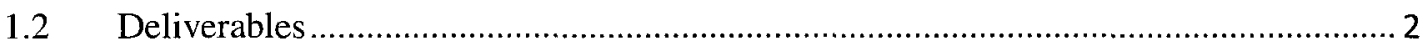

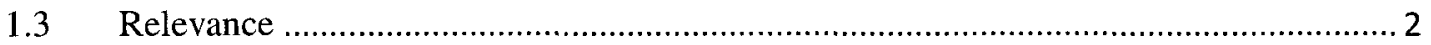

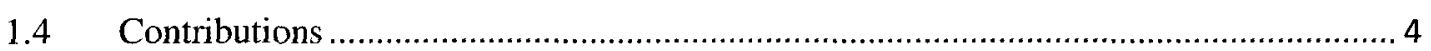

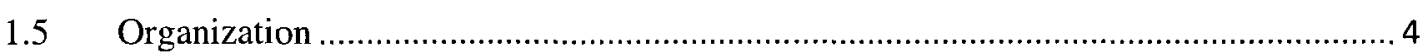

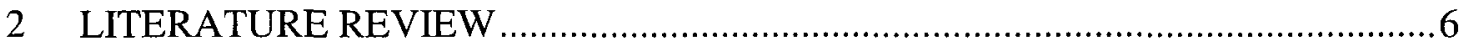

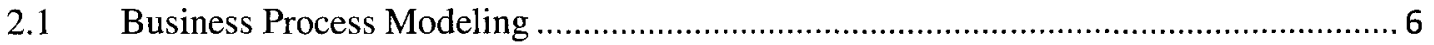

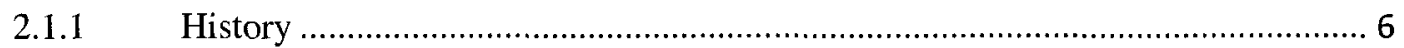

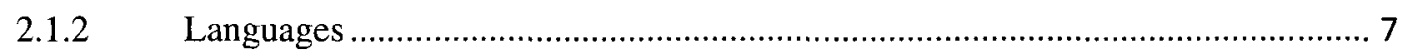

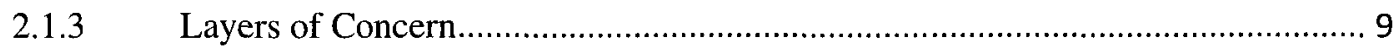

2.2 Using a Business Process Modeling Language ……………………………………..... 11

2.2.1 Capturing Business Processes..................................................................... 11

2.2.2 Business Automation ...................................................................................... 13

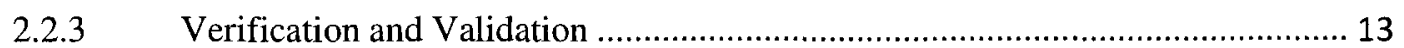

2.2.4 Deployment and Execution.......................................................................... 15

2.3 Quality of Business Process Models ...................................................................... 17

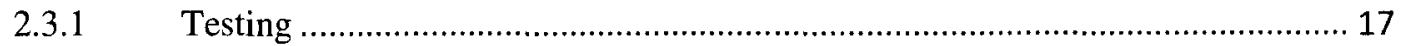

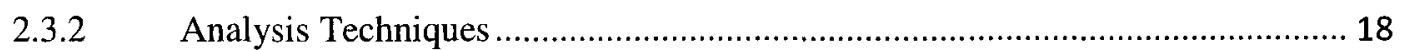

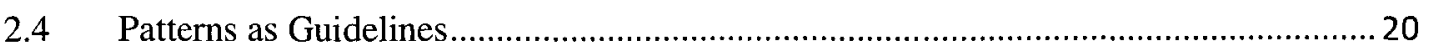




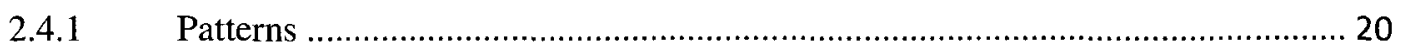

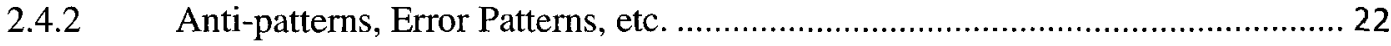

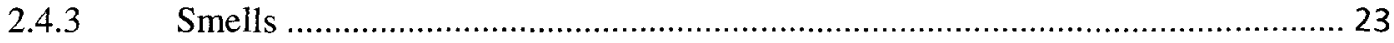

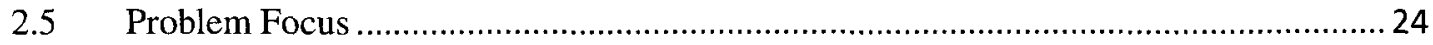

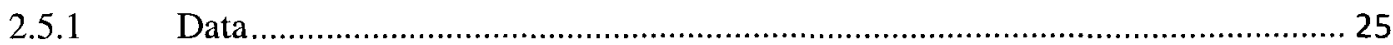

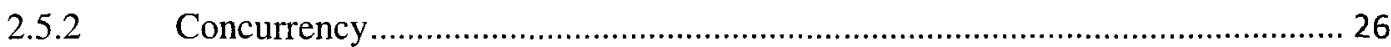

2.5.3 Business process model workflow structure ................................................. 27

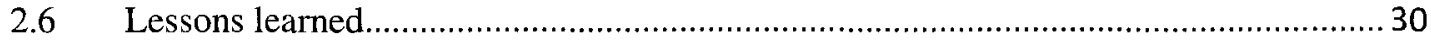

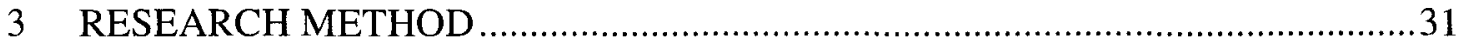

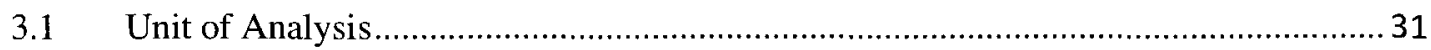

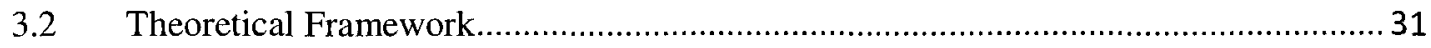

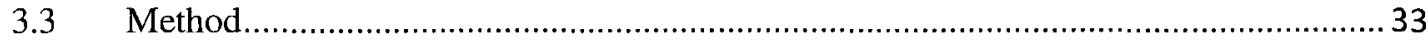

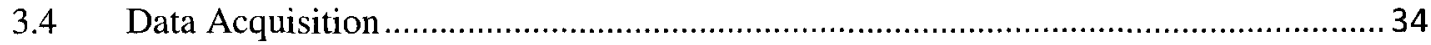

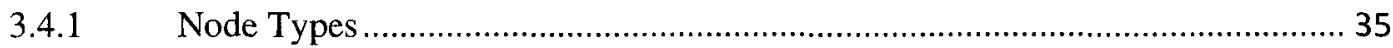

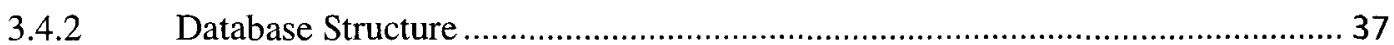

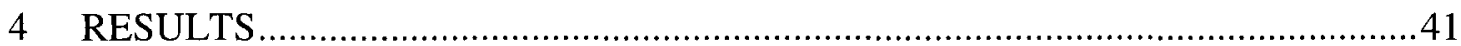

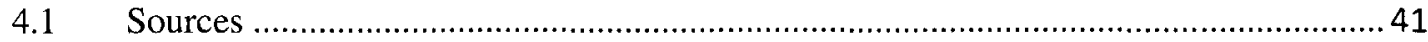

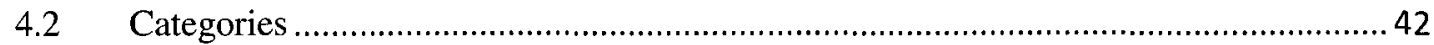

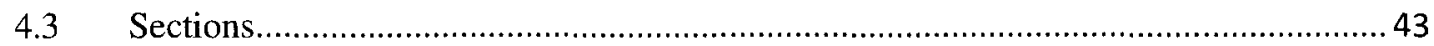

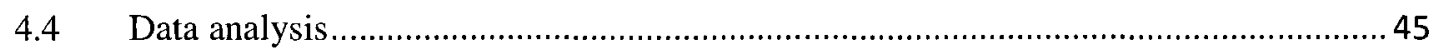

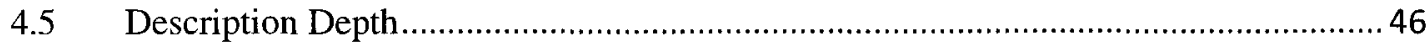

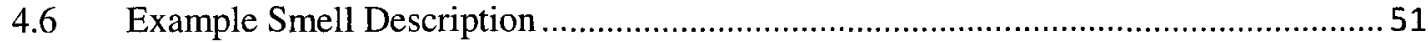

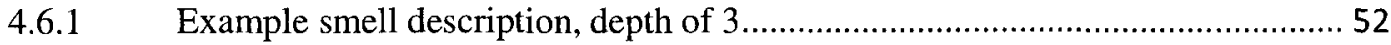

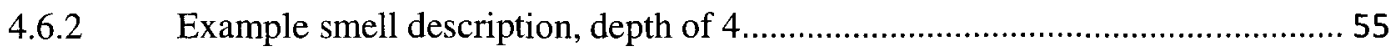

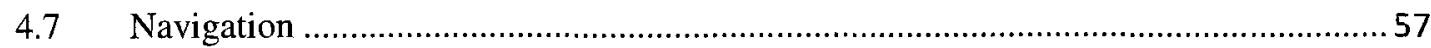

5 CONCLUSIONS, LIMITATIONS, AND FUTURE RESEARCH ........................61

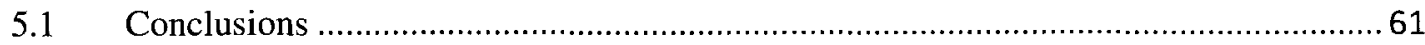

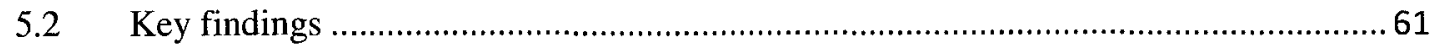

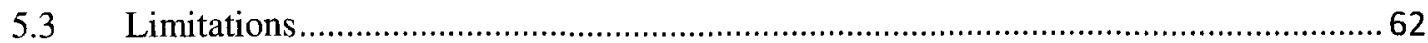




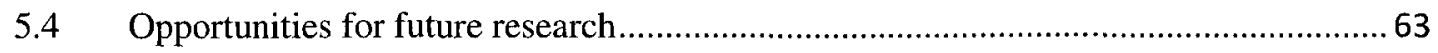

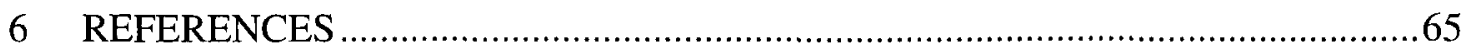

A. DATA CONCURRENCY GUIDELINE LIST ........................................... 72

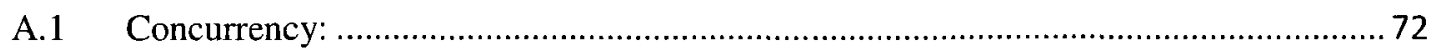

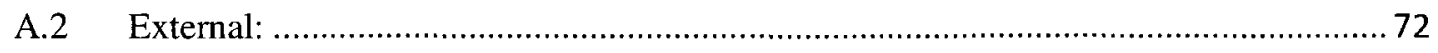

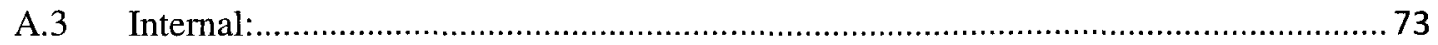

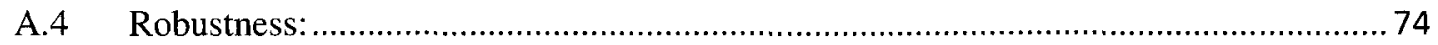

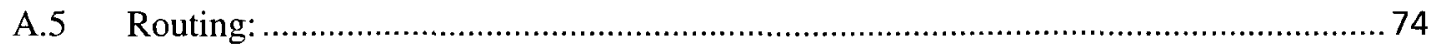

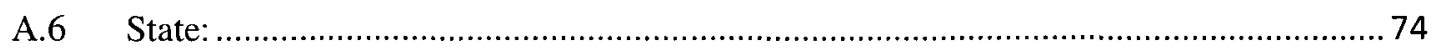

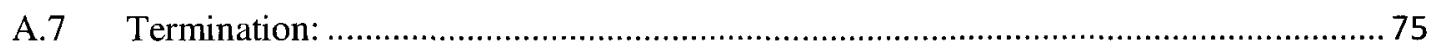

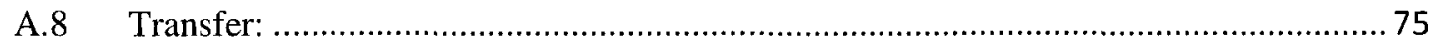

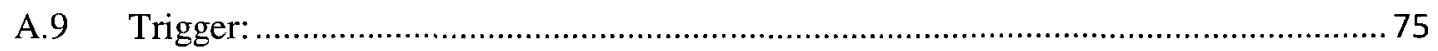

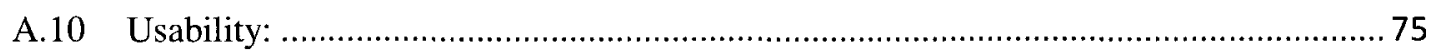

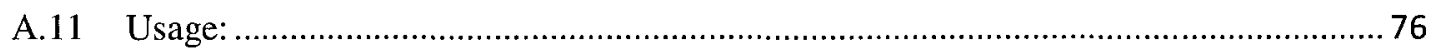

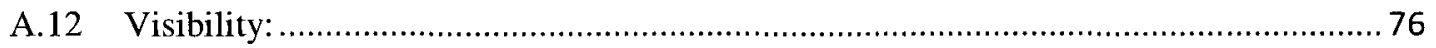

B. DATA CONCURRENCY GUIDELINES AND ISSUES (DEPTH OF 3) ............77

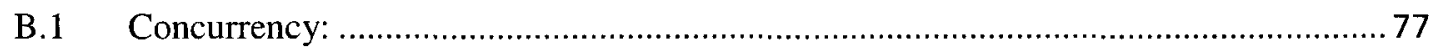

B.1.1 Using a lock when making changes to something shared.............................. 77

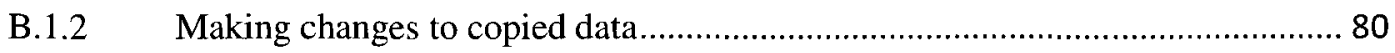

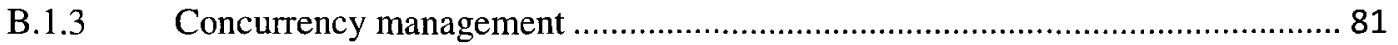

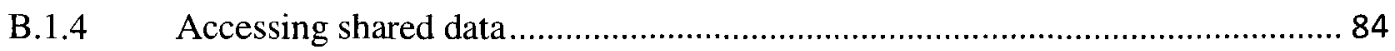

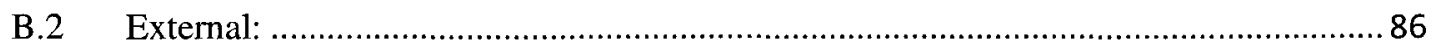

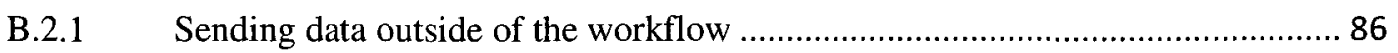

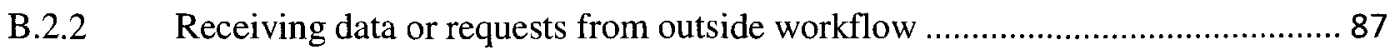

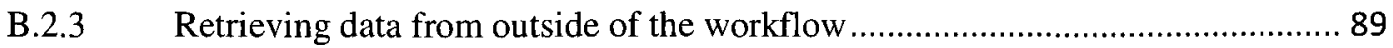

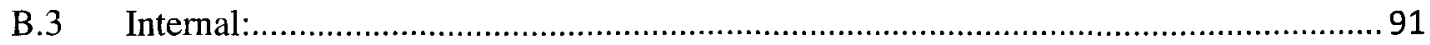

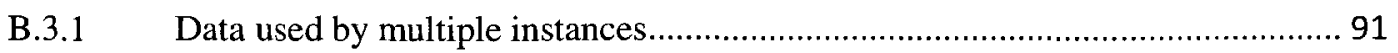

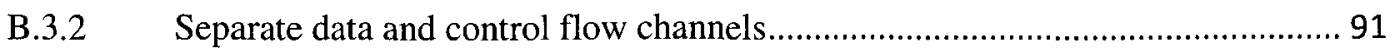

B.3.3 Merging results from multiple instance tasks ................................................ 96 


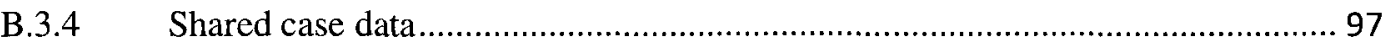

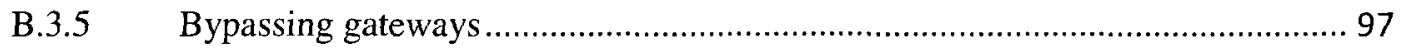

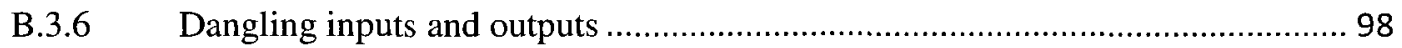

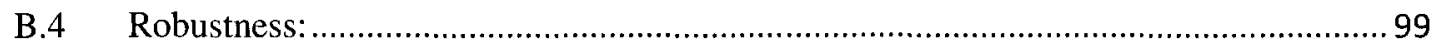

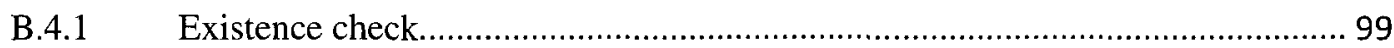

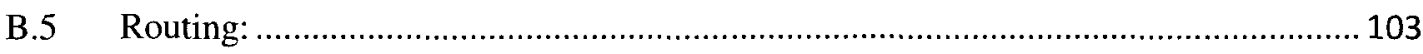

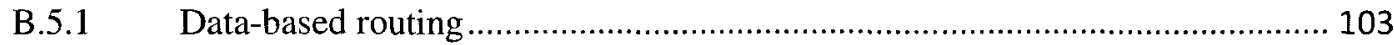

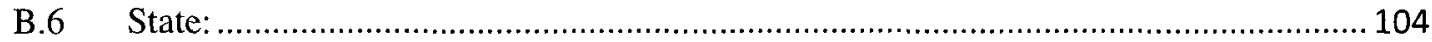

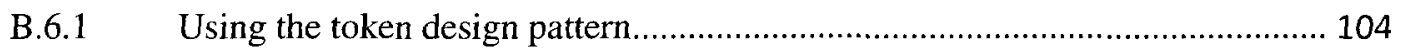

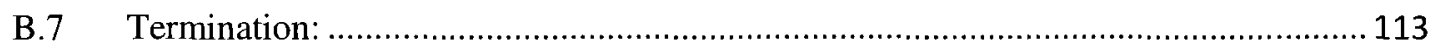

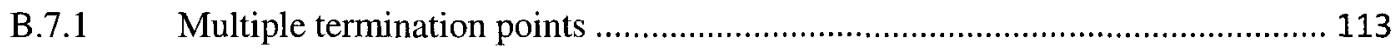

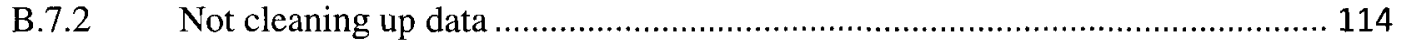

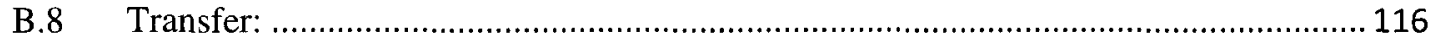

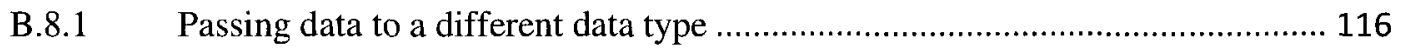

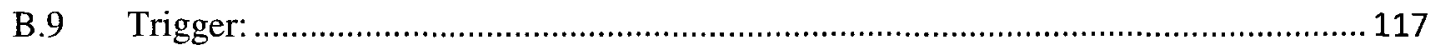

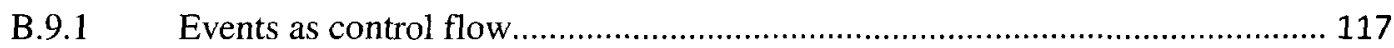

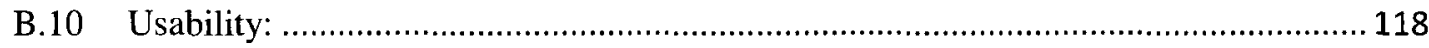

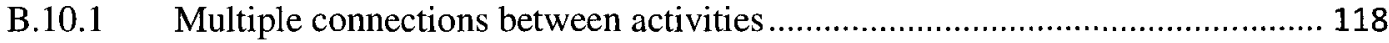

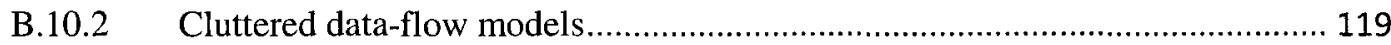

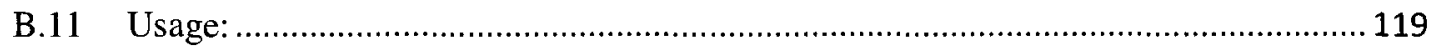

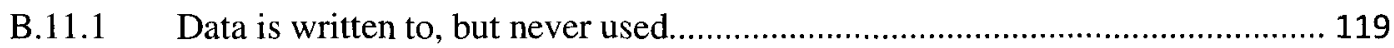

B.11.2 Data is over-written without being used first.............................................. 120

B.11.3 Data is read or destroyed before being initialized or written to........................ 121

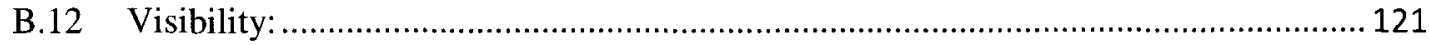

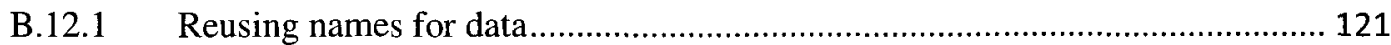




\section{LIST OF FIGURES}

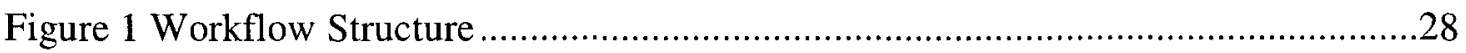

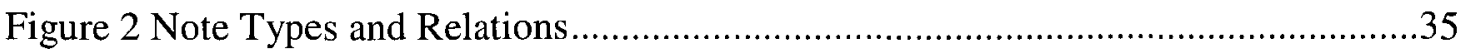

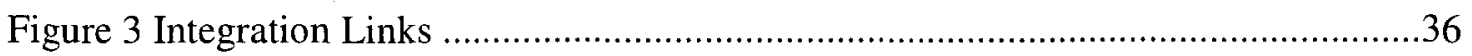

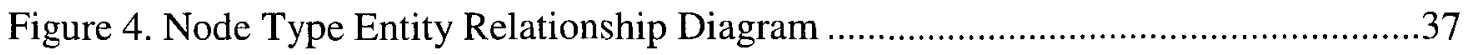

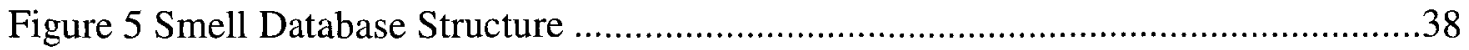

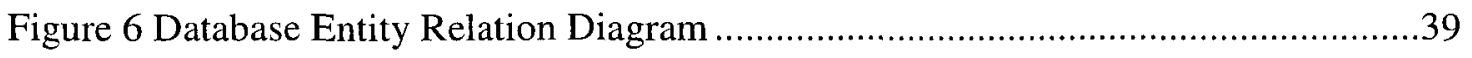

Figure 7 Graphical Representation of Database ...................................................46

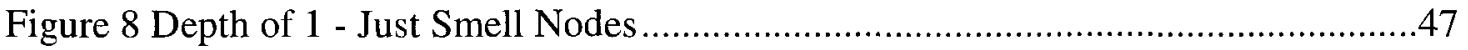

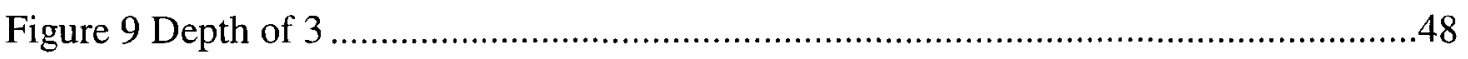

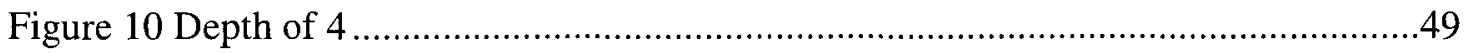

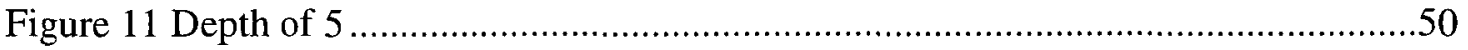

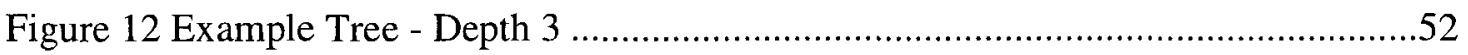

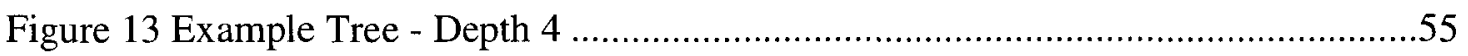

Figure 14 Interactive Guidelines Example .......................................................59 


\section{LIST OF TABLES}

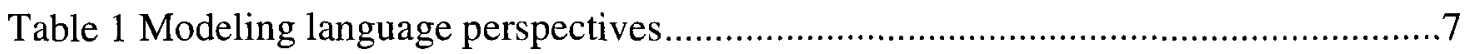

Table 2 Layers of Concern................................................................................. 10

Table 3 Streams about the use of BPM languages.............................................11

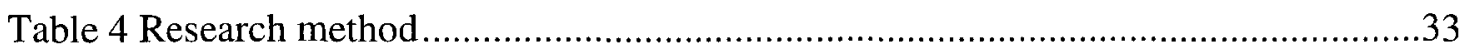

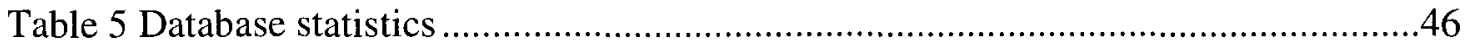

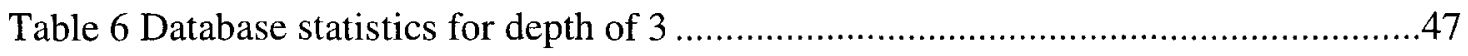

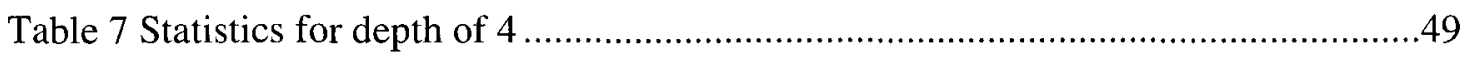

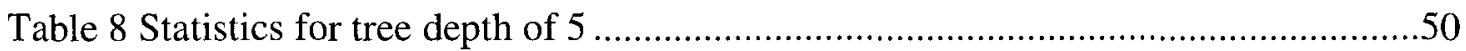

Table 9 Statistics for depth of 3 with similar nodes promoted. .................................51

Table 10 Statistics for depth of 4 with similar nodes promoted ..................................51 


\section{GLOSSARY}

6-Sigma Business management strategy originally developed by Motorola

Atomic task A task which has a self-contained definition and only one instance of the task executes when it is initiated. (van der Aalst et al. 2010)

Block task is a complex action which has its implementation described in terms of a sub-workflow. (van der Aalst et al. 2010)

BPA Business Process Architecture (vs. Automation)

BPEL Business Process Execution Language

BPM Business Process Modeling (vs. Management)

BPMN Business Process Modeling Notation

BPMN-Q BPMN graphical Query language (Laue et al. 2009)

BPR Business Process Re-engineering

Case A process instance (van der Aalst et al. 2010)

CBP Paper used in pattern/smell database: "Concurrent Bug Patterns and How to Test Them", (Farchi et al. 2003)

Components Refers to all of the tasks that comprise a given workflow model. (van der Aalst et al. 2010)

Control channel Supports control flow between tasks. (van der Aalst et al. 2010)

Control flow The order tasks are executed. (van der Aalst et al. 2010)

CORBA Common Object Request Broker (enables distributed software components to communicate with each other) 
CPN Coloured Petri-nets - extension to petri-nets in which every token has a value

DAP Paper used in pattern/smell database: "Data-Flow Anti-patterns: Discovering Data-Flow Errors in Workflows". (Trčka et al. 2009)

Data channel Provides a means of communicating data elements between two connected workflow tasks. (van der Aalst et al. 2010)

Environment The world the workflow operates within. It may contain other workflows, processes or data sources that tasks need to communicate with. (van der Aalst et al. 2010)

EPC Event-driven Process Chain

ITIL Information Technology Infrastructure Library

Lean "Lean" production practice, originally from Toyota

Multi-instance is a combination of the two previous constructs and denotes a task block task that may have multiple distinct execution instances each of which is block structured in nature (i.e. has a corresponding subworkflow). (van der Aalst et al. 2010)

Multi-instance is a task that may have multiple distinct execution instances task running concurrently within the same workflow case. (van der Aalst et al. 2010)

PAP Paper used in pattern/smell database: "Process anti-patterns: How to avoid the common traps of business process modeling" (Koehler et al. 2007) 
Petri-net Graphical mathematical modeling language used to describe distributed systems

PMG Seven Process Modeling Guidelines (7PMG), (Mendling et al. 2010)

PPR Portland Pattern Repository

Process instance An executing instance of a workflow model. There may be multiple cases of a particular workflow model running simultaneously. (van der Aalst et al. 2010)

RPC Remote Procedure Call (for inter-process communication)

SAP Commercial enterprise software vender. Acronym is German for "Systems, Applications and Products in data processing"

SEQUAL "SEmiotic QUALity framework", a systems modeling reference framework for evaluating the quality of models.

(Krogstie et al. 2006)

SME Small/Medium Enterprises

SOA Service-Oriented Architecture

SOAP Simple Object Access Protocol for exchanging information for Web services.

Staffware Commercial process modeling tool suite

Sub-workflow When a block task is started, it passes control to the first task(s) in its corresponding sub-workflow. (van der Aalst et al. 2010)

Task Corresponds to a single unit of work. (van der Aalst et al. 2010) 
Task instance An invocation of a specific task. (van der Aalst et al. 2010)

TQM Total Quality Management

UML Unified Modeling Language

URN User Requirements Notation (Weiss et al. 2005)

VIDE "VIsualize all moDel drivEn programming". (Rech et al. 2007)

WCP Paper used in pattern/smell database: "Workflow Control-Flow Patterns: A Revised View" (Russell et al. 2006)

WDP Paper used in pattern/smell database: "Workflow Data Patterns" (Russell et al. 2004a)

WebSphere IBM WebSphere enterprise software

Workflow Short for workflow model. (van der Aalst et al. 2010)

Workflow A description of a business process in sufficient detail that it is able model to be directly executed by a workflow management system. It is composed of tasks which are connected in the form of a directed graph. (van der Aalst et al. 2010)

WRP Paper used in pattern/smell database: "Workflow Resource Patterns" (Russell et al. 2004b)

XPDL XML Process Definition Language for exchanging business process definitions 


\section{INTRODUCTION}

Business processes have been around as long as there has been commerce, and can involve large numbers of concurrent processors (i.e. people). Likewise, business process modeling can be considered to have begun the first time someone drew a picture to help explain to someone else how their business worked.

Despite this, there is very little literature about verifying Business Process Models (BPM) and where problems may hide, especially when looking for problems with shared data. However, these are well known problems in software engineering, telecommunications, service oriented architectures, etc. Therefore if a large number of people working together can be compared to concurrent processors, then the knowledge from the software engineering world should be applicable to business processes as well.

Business people are not programmers, so this knowledge needs to be intuitive: easy for business people to learn and use. Research in concurrency problems often uses finite state machines and particularly coloured petri-nets. These are not described in a way most business people would understand and are therefore not very usable.

In software, patterns are proven solutions to common problems. Smells are indicators of code that may hide problems and may need to be re-factored (re-designed) using the correct patterns 


\subsection{Objectives}

The objective of this research is to move modeling knowledge from software engineering to the business domain and thus make it available to business process modelers.

\subsection{Deliverables}

The deliverables of the research are:

1. An initial set of guidelines of where to find concurrency-related business process modeling data issues and pointers to possible solutions in literature.

2. A catalog of old and new relationships between smells, issues and patterns, with links back to their original source.

3. Prototype tools for capturing patterns, issues and smells from literature and analyzing their inter-connections.

Note that the guidelines in this thesis are examples of what can be created using this framework. This is due to the amount of software engineering literature that would need to be analyzed and the lack of related work to build upon. The validation of the guidelines is also left as future work. Additionally, while most guidelines suggest solutions, it is up to the modeler to decide which best suits their circumstances, based on the referenced descriptions.

\subsection{Relevance}

This research is relevant to at least three groups. 
1 Top Management Teams need processes to be precisely documented. In a global economy where services can be provided anywhere in the world, the competitive market requires higher quality at a lower cost and cycle time (Przyblek, 2007). Business process modeling is becoming more common to help merge activities around the world. Modeling guidelines will then make it easier to accurately identify and optimize processes and thereby reduce product cycle times. It is far too late to discover there are timing and synchronization problems after a process has been deployed around the world.

2 Business Managers and Analysts are the people who typically design and manage these processes. The ability to find problems and disconnects between different business groups by validating processes and interactions before implementing them across a corporation will improve their chances of success and ability to influence change. (Kazanis \& Ginige, 2002) Even just a document capturing possible problems and how to avoid them will be extremely valuable to them. BPM can also be used for requirements analysis, and to generate testcases (Amyot et al. 2005).

3 Finally, Researchers will find this study useful. Research in Business Process Modeling in general is still relatively new and only covers a small part of the possible issues (Larsen et al. 2005). This thesis also provides a way to look at old knowledge in software engineering in another domain in a novel way which may provide inspiration for further research. 


\subsection{Contributions}

1 In Software Engineering terms, "Patterns" can be described as well-known solutions to common problems. The process of codifying knowledge used in the thesis when applied to patterns makes the following contributions:

a. Patterns are usually organized by the types of solution they provide. Reorganizing this information into "Smells" (i.e. guidelines for detecting situations that may hide errors) provides a novel way to recognize problems in business process models.

b. Breaking patterns from different research sources apart and then recombining them as smells synthesizes new information and reveales relationships between the different sources that were not documented before. This process could be applied to other knowledge domains as well.

2 The proof of concept guidelines help BPM practitioners to improve the quality of their models, through the description of how to avoid/detect data concurrency problems, which were well known in Software Engineering but unfamiliar to business process modelers.

\subsection{Organization}

This thesis is organized into five chapters, and three appendices. Chapter 1 is the introduction. Chapter 2 covers the review of the relevant literature. Chapter 3 presents the method used to conduct the research. Chapter 4 contains the research results. Chapter 5 provides the conclusions, describes the limitations of the research, and identifies opportunities for future research. Appendix A presents a summary list of data 
concurrency guidelines derived from the sample of related literature, and finally appendix B contains guidelines created by drilling three levels into the patterns database. 


\section{LITERATURE REVIEW}

This entire thesis is a literature review about data concurrency issues in business process modeling. Chapter 3 will discuss how the literature was chosen and analyzed, then chapter 4 and the appendices will discuss the results in detail. Therefore, this section will review how this topic fits into the current research.

This chapter is divided into six sections: A brief review of the literature on business process modeling languages in general, and then on how BPM languages are used. The next two sections review first how process models are verified, and then how knowledge from other research areas can be used. The final section discusses lessons learned from the literature.

\subsection{Business Process Modeling}

\subsubsection{History}

Almost every paper on Business Process Modeling, Management, Improvement, or Reengineering has a section on their inter-connected roots. This will not be repeated here, other than to mention BPM's roots are usually traced back to the early 20 th century as a way to improve how organizations work. The 1970s onwards saw the birth of office automation and in the 1990s the growing popularity of Business Process Re-engineering (BPR, as well as TQM, 6-sigma, etc.) all with associated charts and modeling methodologies. (Mendling et al. 2010)

In this century, interest in BPM continues to grow, as does the creation of a multitude of tools and languages. Yet the more formal modeling languages still have not completely 
taken off (Russell et al. 2004a), and most business modeling is still done in tools like PowerPoint, Word, Visio and the like.

\subsubsection{Languages}

Modeling how a business works (or should work) has often involved static diagrams, like fishbone diagrams, pareto diagrams, quality function charts, etc (Kettinger et al. 1997) One of the main reasons interest in BPM languages is growing is that it allows both a consistent way to capture processes and improve the chances of finding problems before new processes are deployed. (Mendling et al. 2010) (Harmon et al. 2010) BPM languages are also becoming able to automate the processes, either directly or through translation.

\begin{tabular}{|l|l|l|}
\hline Role & Perspective & Description \\
\hline What & Functional & What process elements are performed \\
\hline When & Behavioral & $\begin{array}{l}\text { When process elements are allocated (e.g. sequencing), and } \\
\text { how related actions are performed. }\end{array}$ \\
\hline Where & Organizational & $\begin{array}{l}\text { Where and by whom in the organization process elements } \\
\text { are performed. }\end{array}$ \\
\hline Who & Informational & $\begin{array}{l}\text { Entities produced by a process, such as data, documents, } \\
\text { etc. }\end{array}$ \\
\hline How & $\begin{array}{l}\text { Validation and } \\
\text { Verification }\end{array}$ & $\begin{array}{l}\text { How the model was tested to do what it is supposed to do } \\
\text { the way it is supposed to do it. }\end{array}$ \\
\cline { 2 - 4 } & $\begin{array}{l}\text { Modeling } \\
\text { procedure }\end{array}$ & How the model was built \\
\hline
\end{tabular}

Table 1 Modeling language perspectives (from Lin et al. (2002))

Most modeling languages are graphical and can be considered the decedents of flowcharts from the 1970 s and other earlier state diagrams. These languages have different strengths and weaknesses when capturing processes, which Curtis et al. (1992), 
and Lin, Yang and Pai (2002) refer to as perspectives. They divide these into: functional, behavioral, organizational, informational, verification and validation, and modeling procedure. The first four (based on Curtis et al. 1992) capture the "What, When, Where, and Who". Lin et al. (2002) adds "How" the model is built and tested as additional perspectives.

Academic literature contains the largest number of process representations, either in use or just proposed. Petri nets are most commonly used for the underlying model or at least a comparable token based language (Trčka et al. 2009). "A Petri net is a graphical tool for the description and analysis of concurrent processes which arise in systems with many components (distributed systems)." Petri nets are mathematically rigorous, and there is a great deal of research available about using it for process analysis.

In industry literature, BPMN is the most commonly used notation. (Laue et al. 2009) (Harmon et al. 2010) Officially: "The Business Process Modeling Notation (BPMN) is a graphical notation that depicts the steps in a business process. BPMN depicts the end to end flow of a business process. The notation has been specifically designed to coordinate the sequence of processes and the messages that flow between different process participants in a related set of activities"2 BPMN has a token based foundation like Petri nets, but is targeted towards business users and is focused on ease of use. This has lead

\footnotetext{
${ }^{1}$ The graphics, together with the rules for their coarsening and refinement, were invented in August 1939 by the German Carl Adam Petri - at the age of 13 - for the purpose of describing chemical processes Carl Adam Petri and Wolfgang Reisig (2008) Petri net. Scholarpedia, 3(4):6477 http://www.scholarpedia.org/article/Petri_net

2 "BPMN Information". http://www.bpmn.org/Documents/FAQ.htm. Retrieved 2011-01-28
} 
some researchers to be concerned that its lack of mathematical rigor will make some types of errors much harder to find (Börger et al. 2008). As an example, BPMN does not define to what degree it supports multiple tokens, so in the case of synchronizing tokens (N-of-M OR-join) from an earlier multiple choice split (N-of-M OR-split) it does not specify how many levels of splits and joins are supported before there are too many tokens to keep track of. Once tokens are lost, the process will hang waiting for events that can no longer be recognized.

\subsubsection{Layers of Concern}

Business Process Modeling means different things to different people. As part of preparing for this thesis, people who considered themselves as doing business process modeling were spoken to informally. It turned out, the people found worked on a wide variety of modeling endeavors, from high-level business reengineering (e.g. using MS Word as their modeling software) to people working on UML business rules for Service Oriented Architectures, to BPMN-based simulations and finally to paper-based flowcharts for training development.

Just as Lin et al. talked about different BPM languages having different strengths and weaknesses (Lin et al. 2002), Harmon talked about different parts of an organization having different purposes (or concerns) when building models (Harmon 2007). He portrays this as a pyramid to represent the increasing number of people and roles involved from higher to lower levels. This has been greatly simplified in Table 2. 
At the top, at the Organizational Level, modelers want to capture organizational goals and measure/improve the organizations performance and compliance. At the Business Level, management wants to create/capture the processes specific to their projects. Finally at the Implementation level, Human Resource and IT Development must build the infrastructure needed (e.g. training, documentation management, etc.) to make these processes work. The models needed at each level are different in their level of abstraction and functionality.

\begin{tabular}{|l|l|l|l|}
\hline Level & Driver & Focus & Examples \\
\hline Enterprise & $\begin{array}{l}\text { Operations } \\
\text { Research \& } \\
\text { Quality Control }\end{array}$ & $\begin{array}{l}\text { Process } \\
\text { Improvement }\end{array}$ & TQM, 6 Sigma, Lean \\
Business \\
Process & $\begin{array}{l}\text { Management \& } \\
\text { Business Process } \\
\text { Redesign }\end{array}$ & $\begin{array}{l}\text { Organization } \\
\text { Performance \& } \\
\text { Process Redesign }\end{array}$ & BPR, ITIL, BPA ... \\
\hline Implementation & $\begin{array}{l}\text { Information } \\
\text { Technology }\end{array}$ & $\begin{array}{l}\text { Process } \\
\text { Automation }\end{array}$ & $\begin{array}{l}\text { BPMN, SAP, } \\
\text { Staffware ... }\end{array}$ \\
& & & BPEL, XPDL... \\
& & & SOA, Web Services... \\
& & & UML, URN... \\
& & & SOAP, RPC, \\
& & & CORBA... \\
\hline & Hardware & & Computers... \\
\hline
\end{tabular}

Table 2 Layers of Concern (adapted from Harmon (2007))

The focus for this thesis was chosen to be the level where workflow modeling takes place. This is low enough to take advantage of the concurrency lessons learned from Software Engineering in developing SOA, Realtime Systems, etc., but high enough that 
the people doing the modeling are likely coming from a business background and unaware of this knowledge and its applicability to their tasks.

\subsection{Using a Business Process Modeling Language}

\begin{tabular}{|c|c|c|}
\hline Stream & Key highlights of the stream & Key references \\
\hline $\begin{array}{l}\text { Capturing } \\
\text { Business } \\
\text { Processes }\end{array}$ & $\begin{array}{l}\text { - representing business processes in } \\
\text { models } \\
\text { - options as UML, BPMN, other } \\
\text { methods }\end{array}$ & $\begin{array}{l}\text { - Eriksson \& Penker (2000) } \\
\text { - List and Korherr (2005) }\end{array}$ \\
\hline $\begin{array}{l}\text { Business } \\
\text { Automation }\end{array}$ & $\begin{array}{l}\text { using model in the development of IT } \\
\text { / software solutions to improve } \\
\text { business operations }\end{array}$ & $\begin{array}{l}\text { - Chung \& Pak (2006) } \\
\text { - Chowdhary et al. (2006) } \\
\text { - de Castro (2006) } \\
\text { - Nasiri (2004) }\end{array}$ \\
\hline $\begin{array}{l}\text { Verification } \\
\text { and } \\
\text { Validation }\end{array}$ & $\begin{array}{l}\text { - verification / testing of models } \\
\text { - showing the validity / accuracy of a } \\
\text { model - its ability to model the real } \\
\text { world "adequately enough" } \\
\text { - simulate the business process, through } \\
\text { iterations of the models, through time }\end{array}$ & $\begin{array}{l}\text { - Chowdhary et al. (2006) } \\
\text { - Fishwick (2007) } \\
\text { - Hseuh et al. (2008) }\end{array}$ \\
\hline $\begin{array}{l}\text { Deployment } \\
\text { and } \\
\text { Execution }\end{array}$ & $\begin{array}{l}\text { - methods and importance of } \\
\text { implementing what has been } \\
\text { developed or learned } \\
\text { - includes distribution, training, } \\
\text { measurement, and maintenance } \\
\text { aspects as well }\end{array}$ & $\begin{array}{l}\text { - Chung \& Pak (2006) } \\
\text { - Chowdhary et al. (2006) } \\
\text { - Machado et al. (2005) } \\
\text { - Nasiri (2004) } \\
\text { - Hseuh et al. (2008) } \\
\text { - Kazanis \& Ginige (2002) } \\
\text { - Przyblek (2007) }\end{array}$ \\
\hline
\end{tabular}

Table 3 Streams about the use of BPM languages

There is a great deal more literature on how to capture business processes and how they are applied in different industries than there are papers on verification and validation, 
showing that this is an area often overlooked. This section is divided into four streams: Capturing Business Processes, Business Automation, Verification and Validation, and finally Execution and Deployment.

\subsubsection{Capturing Business Processes}

In order to discuss how to model a business process, you must first understand what a business process is. For example, one of the key works on UML for BPM is by Eriksson and Penker (2000). They built upon the business process foundations of Davenport, Hammer and Champy in the early 1990s, and derived the following definition of a business process. A business process is something that (Eriksson \& Penker, 2000):

$1 \quad$ Has a goal.

2 Has specific input.

3 Has specific output.

4 Uses resources.

5 Has a number of activities that are performed in some order, depending on conditions and events that occur during the execution of the process. The activities within the process can be seen as subprocesses.

6 Affects more than one organizational unit. It is horizontal rather than vertical in regard to the traditional organization of the business.

7 Creates value to some kind of customer. The customer can be either internal or external to the business.

As with many researchers, List and Korherr (2005) built upon the work done by Eriksson and Penker, and they describe how they have customized the Eriksson-Penker UML 
Extensions in their environment. List and Korherr offer a useful summation of what the requirements of a business process are, in order to be able to model it. This includes the ability to represent activities, roles, customers, process owners, process types, deliverables and measures.

\subsubsection{Business Automation}

Nasiri (2004) points out that "Rarely there is a business system capable of being fully automated. In other words some parts of a business system always remain manual because of their nature. The intelligence to recognize which parts should be automated and which should be left intact is an artistic activity far beyond current methods."

BPMN is able to generate BPEL to assist with process automation. Other approaches to automating model based business processes are also mentioned in the literature: De Castro (2006) discusses in her paper the stages in which a UML process model can be translated into a web services composition language for generating web based applications. Chung et al. (2006) used it to create a knowledge-based support system (i.e. expert system). Chowdary et al. (2006), describes automatically generating code from UML diagrams to capture business performance metrics.

\subsubsection{Verification and Validation}

It is important to ensure that the business process model is correct. Research in this stream of literature discusses the verification and validation requirements of a given model or system. Verification and validation $(\mathrm{V} \& \mathrm{~V})$ are key steps to ensuring that the 
modeling effort is successful and often ignored or given lip service. As mentioned above, the majority of modeling articles spoke little of the validation and verification.

This subsection talks about the purpose of the different phases of checking a process' quality. Section 2.3: "Quality of Business Process Models" discusses in more detail the different ways this can be accomplished.

\section{Verification}

Verification is the process of testing truth against requirements, or more specifically, a model is verified that it is built correctly against how it was intended / specified (Fishwick, 2007), i.e. free of bugs / errors / omissions. This is often associated with testing of an executable application but is also necessary for manual processes. Verification is something that must be considered throughout the development of a product, process, model or system. Methods of verification continue to be refined, and advanced modeling and simulation environments often offer automated or assisted means of verification. Hseuh et al. (2008) describe their use of the Object Constraint Language to automatically verify their process models.

\section{Validation}

Validation is the process of testing system behaviour against empirical observations (Fishwick, 2007), i.e. does the system adequately represent the real world? Validation of models or systems is often neglected, and a system with attractive graphics and appeal, can easily distract from validating that a given model is actually accurate. Chowdhary et 
al. (2006) particularly noted that they experienced difficulty with model validation when dealing with complex systems. Hseuh et al. (2008) found that it was effective to have the end-users of a process use a simulation environment to validate if the models were realistic. While it is generally noted that validation is an important step, few of the initially reviewed papers made mention on how it was done, or if it was done at all.

\subsubsection{Deployment and Execution}

Once business processes have been correctly captured, optimized, validated, and verified, they need to be reintroduced into the business environment either through paper or automated tools. These tools need to be deployed, the people involved need to be trained, and conformance needs to be tracked. "Distribution, which is going to be a common feature of many enterprises, is very seldom taken into account while modeling, which in turn hinders the success of modeling approach". (Nassiri 2004)

\section{Distribution}

Kazanis \& Ginige (2002) discuss that different sized businesses will use and deploy BPMs differently. "Big Business" will use models to develop large scale projects and have the resources to go through a massive development phase with large amounts of process automation, followed by a massive training phase and then be able to provide a massive amount of support to keep everything working. In contrast, Small Medium Enterprises (SMEs) have time and budget constraints. They may only make improvements to existing processes which are most likely paper based. Training and support would be provided through word of mouth as opposed to training material. 


\section{Training}

Business process modeling languages are not always easy to understand, or instead lack too much information to be automated. BPMs based on UML allow BPM experts to talk the same "language" as software experts making it easier to capture design processes, and for the software professionals to automate those processes in turn. It also makes it easier for software engineers to learn new processes when they are introduced. (Przybylek 2007) Ease of understanding is even more important when businesses span the globe and everyone does not speak the same language. Process mistakes can cause costly errors in both time and money.

Both Hsueh et al. (2008) and Machado et al. (2005) mentioned using model driven simulations (or animations) to help people unfamiliar with the modeling language validate if processes had been captured correctly. Hsueh et al. (2008) takes this further and uses the simulation as a way to train new participants, and found that people actually liked learning using a simulator. This proved successful enough in their experiments to be given permission to go to a trial deployment within the financial institution where they were working.

\section{Measurement}

As the adage goes, "If you can't measure it, you can't manage it". For process improvement to be effective it is necessary to be able to determine if a new process is being followed and is achieving the desired results. While Chowdhary et al. (2006) discuss automatically generating Business Performance metrics from architectural UML 
diagrams, this technique should also be applicable to measuring automated Business Processes captured in UML. It is not obvious from Chowdhary's article if IBM has actually done this. The article does mention that IBM's tools automate creating the code to capture these metrics without needing programmers.

\subsection{Quality of Business Process Models}

The section above discussed the importance of Business Process Modeling, how it applies throughout and organization's structure and how it may be used. Quality then becomes of growing importance in order to reduce the chances a mistake may not be found until a process is implemented (potentially around the world), as well as reducing the accompanying economic cost.

\subsubsection{Testing}

When a business process is fully automatable, then it can be tested similar to a software program and one can possibly use the model to generate testcases to use on the final implementation (Amyot et al. 2005). However, as Nasiri et al. (2004) point out above, business models are rarely fully automatable. Fortunately, modeling tools often provide the ability to test the model through simulation (Hsueh et al. 2008).

Since it is unlikely to be practical (or even probable) to test every possible execution pathway through the model, then testers have to use experience to know where to look for problems, or depend on tools to look for them (if such tools exist). 


\subsubsection{Analysis Techniques}

Over the last 20 years researchers have been working on tools to analyze the quality of models based on structural metrics. Van der Aalst et al. (2008) provide an overview of these techniques, but also point out that most of these are based on AND/XOR graphs or the equivalent control flow in more sophisticated modeling languages (e.g. Petri-nets, BPMN, EPC, etc.).

In particular, van der Aalst et al. mention three techniques: Coverability graphs, which indicate whether the entire graph is reachable, as well as potential deadlocks and infinite loops. Invariants, where the absence of certain place and transition invariants indicate possible problems. Finally, there are Reduction rules, which simplify the net without removing important structure so the other approaches can execute in a reasonable amount of time.

Mendling et al. (2008) demonstrate using reduction rules to analyze 600 publically available SAP reference models, showing 34 of them contained errors. Laue and Awad (2009) use a graphical query language (BPMN-Q) on 109 models from a public repository to find BPMN model fragments that match structural error patterns (which can be considered a reduction like approach) and found 14 erroneous models.

\section{Analysis problems}

There are also concerns with the analysis approach. Koehler and Vanhatalo (2007) point out that the success of most business processes are measured by economic indicators such as cost and profit, and that structural analysis tools can only provide "insights" that 
problems may be present. Even worse, these tools' results can be misleading by reporting issues that are not important while completely missing others that are.

Koehler's and Vanhatalo's paper (2007) mentions they were not able to find very many papers which address the measurement of quality in process models. A number of papers refer to the Guidelines of Modeling (GoM) framework (Becker et al. 2000), which defines six "guidelines", correctness, relevance, economic efficiency, clarity, comparability, and systematic design. The SEQUAL framework (Krogstie et al. 2006) lists: physical, empirical, syntactic, semantic, pragmatic, social, and organizational quality types. Finally, in the ISO/IEC 9126 Software Product Quality Model, the quality of process models is based on functionality, reliability, usability, and maintainability. (Guceglioglu \& Demirors, 2005) While problems related to attributes like correctness may be derived by analyzing the model, other attributes, like clarity and economic efficiency are more subjective.

Mendling et al. (2010) point out that the level of modeling competence that many casual modelers have is notoriously low. Frameworks like SEQUAL and Guidelines of Modeling tend to be too abstract for non-experts to use, and the various collections of hints lack the sound research to back them up.

Finally, analysis tools are not available for all Business Process Modeling languages. Several studies show small to medium size companies often use "paper based" process modeling due to not having the time and resources for people to become proficient at using formal languages and tools (see Kazanis et al. (2002), Davies et al. (2006)). 


\subsection{Patterns as Guidelines}

As mentioned above, modeling tools are mostly used in large companies (Kazanis et al. 2002) (Davies et al. 2006), and even then business modelers may not have much software engineering (or mathematical) experience with modeling. Guidelines are needed to help them learn the best techniques to do their work.

Two of the better known frameworks: Guidelines of Modeling (GoM), which is inspired by general accounting rules (Becker et al. 00), and SEQUAL, which stands for the "semiotic quality" framework (Krogstie et al. 2006), have proven valuable, but both require a minimum level of expertise to understand and use.

Mendling et al. (2009), in their paper "Seven Process Modeling Guidelines (7PMG)", provided an overview of the types of guidelines available in business process modeling research and noted the need for more pragmatic and easy to understand guides. They analyzed the available empirical studies and provided a list of seven guidelines backed by research.

These problems have existed in software engineering for decades, and while coding guidelines are still important for consistency, the "best ways" to solve and/or find problems are described in the next three sections: Patterns, Anti-patterns (etc.) and Smells.

\subsubsection{Patterns}

Patterns are used in software engineering to describe reusable solutions for common problems. This definition appears in multiple places (e.g. Laue et al. 2009). Patterns are 
usually captured in a semi-formal format that describes the "solution to a problem in a context" (Lea 2000). Collections of related patterns are sometimes referred to as "pattern languages". For the purpose of this thesis, patterns are essentially guidelines for ways to solve a problem under specific conditions. They are typically not tied to any programming or modeling language.

Patterns originally came from the world of architecture (e.g. buildings), as reusable descriptions in books by Alexander et al. (1977, 1979). At OOPLSA'87, Kent Beck and Ward Cunningham presented their work applying this idea to programming (Cunningham \& Beck, 1987). Software patterns gained in popularity when Erich Gamma, Richard Helm, Ralph Johnson and John Vlissides (aka "The Gang of Four") published the book "Design Patterns: Elements of Reusable Object-Oriented Software" in 1994. Since then, there has been a plethora of papers, books and websites, describing patterns in all areas of design and architecture (not just software, or buildings). More information and references can be found in the Patterns Discussion FAQ (Lea 2000).

For Service Oriented Architecture (SOA) and Web Services and lower layers of concern (section 2.1.3), there is an abundant source of pattern languages and repositories ${ }^{3}$, many of which contain patterns related to implementing business process logic. Within the workflow modeling community the early major repository of patterns was the Workflow Patterns initiative (van der Aalst et al. 2010) created by WMP van der Aalst and AHM ter Hofstede, primarily as a way to compare workflow modeling tools and languages. The 20

\footnotetext{
${ }^{3}$ and catalogues of repositories. See http://hillside.net/patterns/patterns-catalog, and http://www.dmoz.org/Computers/Programming/Methodologies/Patterns_and_Anti-Patterns
} 
patterns documented in their paper (van der Aast et al. 2003) have been reused in many other papers to introduce or analyze modeling languages. These patterns have expanded to far over a hundred, 126 of which were analyzed as part of this thesis. (Russell et al. 2004a, 2004b, 2006)

\subsubsection{Anti-patterns, Error Patterns, etc.}

While patterns captured common solutions to common problems, they also began to be used as a way to describe common bad solutions as well. Andrew Koenig coined the term "anti-patterns", inspired by the Gang of Four's book (Gamma et al. 1994) and described it as: "An anti-pattern is just like pattern, except that instead of solution it gives something that looks superficially like a solution, but isn't one" (Koenig 1995). The term was made popular by the book "AntiPatterns: Refactoring Software, Architectures, and Projects in Crisis" (Brown et al. 1998). This book dealt a great deal with aspects of bad management, which actually places it into the management and organizational layers of concern (from section 2.1.3), which is above the level this thesis is focusing on.

Lists of anti-patterns are only one source of common problems. The VIDE Consortium did an exhaustive survey of the literature up to 2007 on quality defects in model driven software (Rech et al. 2007) of approximately 950 papers. From this they created a list of related topics: "Anomalies, Anti-guidelines, Anti-patterns, Bug Patterns, Bug Taxonomies, Critic Rules, Defects, Defect Patterns, Design Bugs \& Errors, Error Patterns, Fault Patterns, Flaws, Heuristics, Illnesses, Metric Thresholds, Negative Patterns, Pitfalls, Design Principles, Puzzles / Puzzlers, Design Rules, Code Sins, Styles, Conventions, and Rules" which they document in their report. (Rech et al. 2007) 
For workflow models, most papers list the first categorization of error patterns as having been done for deadlock patterns by Onada et al. (1999) at the University of Osaka. Liu and Kumar (2005) discuss how unstructured workflows can be mapped into structured ones, and name the combinations of splits and joins that can lead to control flow errors. Koehler and Vanhatalo (2007) report on lessons they learned reviewing hundreds of process models created in IBM Websphere, as well as few others. All of these are primarily based on control flow.

\subsubsection{Smells}

...smells represent problematic parts of the software system that seem wrong, complicated, or cumbersome to an experienced developer. In general, smells are problems that are associated with one or more specific refactorings (i.e., concrete treatments) that might be applied to remove the smells. (Rech et al. 2004)

Software smells (unlike Anti-patterns, Error patterns, and others above) do not claim that there is a problem in the code, only that based on experience a senior programmer would be worried that the code may hide problems, be hard to verify, or be hard to make changes to in the future.

The term was coined by Keith Beck in the Portland Pattern Repository [PPR] and made popular in the article "Bad Smells in Code" (Fowler et al. 1999). As the quote above mentions, in general smells are associated with refactoring code (i.e. ways to rewrite/improve/simplify it) in order to improve its testability and maintainability. Code can also become smellier over time, i.e. as more changes are made to code it may become 
less maintainable and therefore its smell may give the maintainer hints at how to best refractor it.

There are now a number of lists of smells. The VIDE D4.1 report references several lists (Rech et al. 2004), as does the Portland Pattern Repository [PPR]. Mäntylä (2003) improved the categorization of Fowler's original list to make it easier to use.

In modeling, there are a number of tools which use templates (along the lines of BPMNQ above) (Laue et al. (2009), Gruhnet al. (2009), Braem et al. (2006), etc), or structural metrics (Mendling et al. (2007), Held et al. (2008), etc) to find smells automatically. While structural metrics (like the number of branch points) may or may not be obvious problems to an experience modeler, they do indicate the potential for problems and are therefore smells. All of these primarily deal with control flow, and if they are not included in a modeler's design tool (if they even have one) they are then of limited value.

The only paper found close to this thesis' topic was "Refactoring Process Models in Large Process Repositories" by Weber and Reichert (2008). They created a list of eleven refactoring techniques for process models based on software engineering research. Unlike this thesis, they focus on how to fix problems, while this thesis focuses on how to find them. They also limit themselves to control flow, while our focus is data flow, specifically with regards to concurrency.

\subsection{Problem Focus}

There are an immense number of patterns and associated problem types that guidelines could be built for (e.g. Rech et al. (2007)), which would take far more time and space 
than available. As mentioned above, most research focuses on control flow. There is also a lot of information on resource patterns hidden within project management literature.

Therefore this thesis focuses on the convergence of two types of problems that have not been widely studied in BPM: Data and Concurrency, which are discussed in the next two sub-sections. The third sub-section provides common terminology from the literature to describe BPM workflow structures.

\subsubsection{Data}

Data flow problems occur when values are: over-written un-intentionally, not visible where needed, cause the wrong control flow path to be taken, etc. (See Section 4: RESULTS for a longer list)

Most of the literature about workflow patterns (or anti-patterns, etc.) dwells primarily on control flow. Russell et al. (Russell et al. 2004a) in the largest collection of workflow data patterns (and one of the only) mentions when commenting on existing tool offerings: "One of the most immediate [observations] is that the level of direct support for data patterns in standard workflow design tools is minimal."

The other data flow papers focused on tools or algorithms to find problems. These papers also mentioned the lack of data flow research in business processes.

- Meda et al. (2010) list verification algorithms for data flow, but comment: "Existing workflow systems that model business processes, [...] are generally based on control 
flow modeling, but usually also allow input and output data elements to be specified. However, data flow errors are not typically verified."

- Trčka et al. (2009) give a list of the current research on dataflow analysis, but point out: "Despite the abundance of analysis techniques to discover control-flow errors in workflow designs, there is hardly any support for data-flow verification. Most techniques simply abstract from data, while data dependencies can be the source of all kinds of errors."

- Sundari et al. (2007) point out: "While a number of analytical and algorithmic methods exist for verifying control-flow correctness, relatively few schemes are available for verifying data-flow correctness."

\subsubsection{Concurrency}

Bugs in concurrent programs can be difficult to find and reproduce (Barland et al. 2005), and as stated before, business processes may involve hundreds of individual human "processors" working in parallel. Solutions of how to deal with concurrency problems (e.g. deadlock, race conditions, etc.), and the issues that arise when applying those solutions (e.g. live-lock, starvation, etc.) are common in software engineering, telecommunications, etc., but not well documented for business processes.

Petri nets, which are often used in academia to study process models (Trčka et al. 2009), were invented to deal with parallelism. As mentioned above, most of the research into process modeling has focused on control flow issues like deadlocks (Meda et al. 2010). Russell et al's (2004a) collection of BPM data patterns list problems with contention for shared data and how to avoid it by serialization, but does not go further and discuss the 
problems with serialization. While this knowledge is reasonably well know to software engineers at lower layers of concern, it is not reasonable to expect business-focused modelers (using commercial tools or not) to be aware of this.

Trčka et al. and Russell et al. both have commented on this:

"In the field of software verification, static analysis of software and model checking have been successfully used to discover program bugs that are caused by, e.g., non-initialized or dead variables. In this, totally different, application domain, concurrency issues are rarely treated and systematic classification of errors is missing." (Trčka et al. 2009)

"Another observation is that many systems/standards do not offer support for multiple instance tasks. A number of the offerings examined implement a shared repository for data elements (at block, scope or case level) where tasks access a common set of data elements rather than passing them between tasks." (Russell et al. 2004a)

\subsubsection{Business process model workflow structure}

As much as possible, this thesis tries to avoid technical jargon with which the people doing business process modeling may not be familiar. However, in the list of guidelines, there will sometimes be the need to be precise and for that the definitions adapted from van der Aalst's and ter Hofstede's Workflow Patterns Home Page (van der Aalst et al. 2010) (Russell et al. 2004a) will be used.

A workflow or workflow model is a description of a business process in sufficient detail that it is able to be directly executed by a workflow management system. 
A workflow model is composed of a number of tasks which are connected in the form of a directed graph.

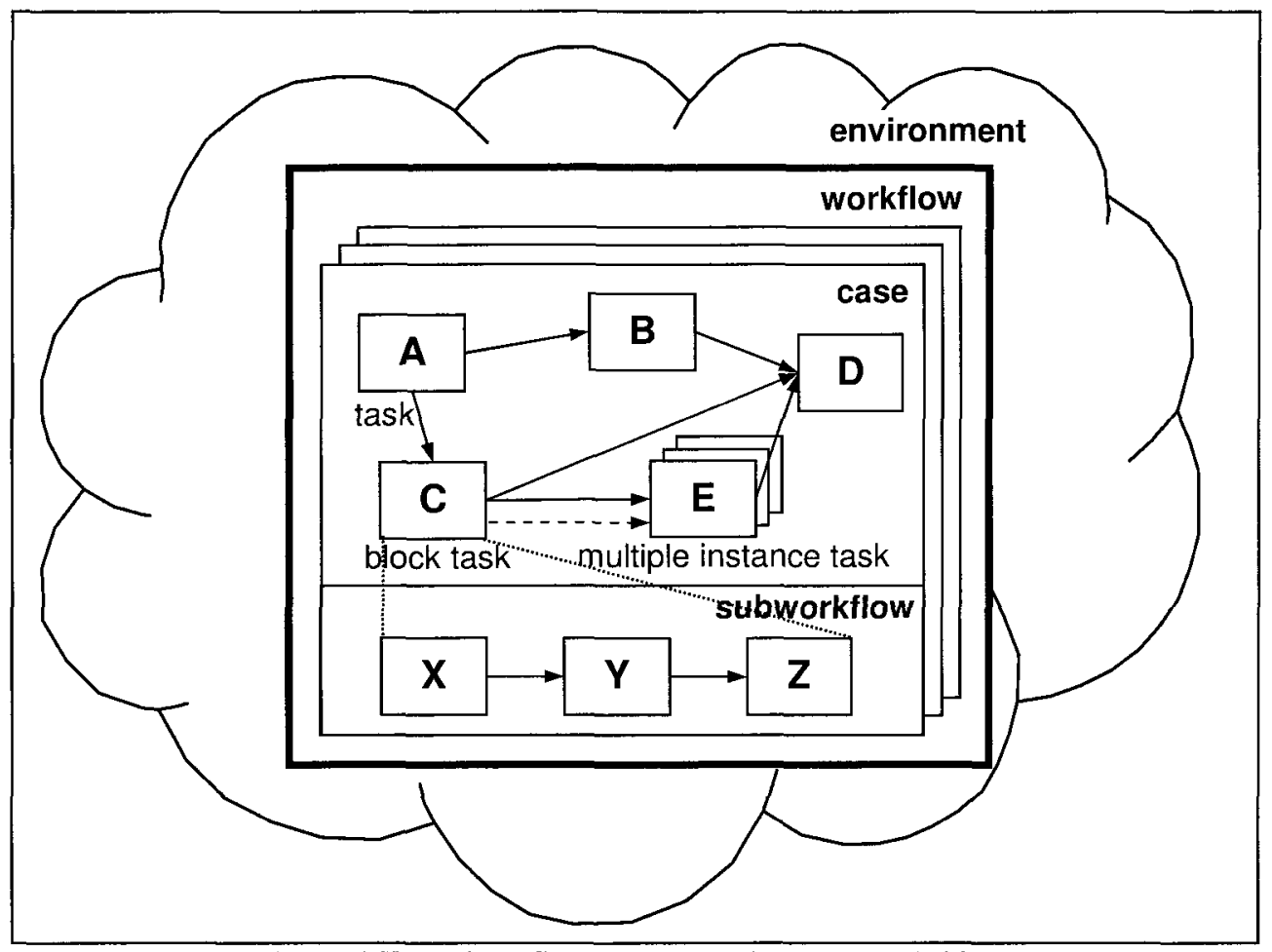

Figure 1 Workflow Structure (van der Aalst et al. 2010)

The environment is the world the workflow operates within. It may contain other workflows, processes or data sources (e.g. people) that tasks need to communicate with.

A case or process instance $i$ an executing instance of a workflow model. There may be multiple cases of a particular workflow model running simultaneously, however each of these is assumed to have an independent existence and they typically execute without reference to each other.

A task instance is an invocation of a specific task. A task instance may initiate one or several task instances when it completes. This is illustrated by an arrow from the completing task to the task being initiated e.g. in Figure 1, task instance B is initiated when task instance A completes. This may also occur conditionally. 
A task corresponds to a single unit of work. Four distinct types of task are denoted: atomic, block, multi-instance and multi-instance block. We use the generic term components of a workflow to refer to all of the tasks that comprise a given workflow model.

An atomic task is one which has a simple, self-contained definition (i.e. one that is not described in terms of other workflow tasks) and only one instance of the task executes when it is initiated.

A block task is a complex action which has its implementation described in terms of a sub-workflow. When a block task is started, it passes control to the first task(s) in its corresponding sub-workflow. This subworkflow executes to completion and at its conclusion, it passes control back to the block task, e.g. block task $C$ is defined in terms of the subworkflow comprising tasks, $X, Y$ and $Z$.

A multiple-instance task is a task that may have multiple distinct execution instances running concurrently within the same workflow case. Each of these instances executes independently. Only when a nominated number of these instances have completed is the task following the multiple instance task initiated.

A multi-instance block task is a combination of the two previous constructs and denotes a task that may have multiple distinct execution instances each of which is block structured in nature (i.e.has a corresponding sub-workflow).

The control channel supports control flow between tasks, which is illustrated in Figurel by the solid arrows between the tasks.

The data channel provides a means of communicating data elements between two connected workflow tasks. This may be combined with the control channel or may be distinct. In Figure 1, a distinct data channel is represented as the dashed line between task instances $C$ and $E$.

(van der Aalst et al. 2010) 


\subsection{Lessons learned}

- Business processes suffer from the same problems as distributed computer systems, even when the work is being done by humans

- There is an immense amount of research about data concurrency problems in computer science, but very little corresponding research for business processes

- There is very little research about "smells" as well but there is a lot of related research that can still be used

- If business processes have the same problems as distributed computer systems, then the same strategies to find and prevent these problems should be applicable to business processes as well. 


\section{RESEARCH METHOD}

This thesis uses a constructive approach by building a framework with which to analyze software engineering research in data concurrency problems and convert it into guidelines useable for business modelers to recognize problems.

This chapter describes the methods used to produce the deliverables of this research. It is organized in four sections. Section 3.1 describes the unit of analysis. Section 3.2 describes the theoretical framework. Section 3.3 identifies the research method. Finally, section 3.4 describes how the data was analyzed.

\subsection{Unit of Analysis}

The unit of analysis was data concurrency pattern descriptions from which issues and solutions were derived. Initially known BPM patterns were used, but the search was expanded into other areas of research, such as Software Engineering. Other sources of problem descriptions, such as those discussed in section 3.4 "Data Acquisition" below, were also investigated.

\subsection{Theoretical Framework}

Bouhours et al. (2009) described a Design Pattern as "the optimal reusable microarchitecture for a type of problem" and Fowler et al, (1999) states "Smells are any symptom that possibly indicates a deeper design problem". Therefore this thesis" "guidelines for finding problems" topic could be referred to as "Smells" in Software Engineering. Bouhours et al. (2009) also show in their paper that an inappropriate or 
poorly implemented pattern can be considered a Smell as well. This was used to create a library of smells from pattern and related research. (See section 3.4 below.)

Linda Rising created a Patterns Almanac in 2000 containing summaries of documented patterns up to that date, with links to where the full description can be found. The format is sometimes referred to as a "patlet" (Cunningham 2005), and specifically the format as described in the PatternForge wiki is used:

"A good patlet is short, only about 2-3 sentences. It distills the pattern to its essence. Here, we extend the basic patlet format to include links to related patterns. Each link should document the reason for applying the other pattern." (Weiss 2007a).

A theoretical framework provides a lens through which to look at the research problem. The concept of "smell" provides a lens through which to look at patterns, and Rising's framework provides an initial template of how to organize patterns and their relationships. To that framework the notion of smells has been incorporated

Rising's format is extended slightly further by breaking patterns into 3 pieces: Issues, Solutions, and the Pattern implementation, and then finally creating Smells for the Issues. This increases information richness of the linkages and is described in more detail in section 3.4.1 below.

Mäntylä (2003) shows that smells need to be categorized to be more usable, which has been added as well. 


\subsection{Method}

Table 4 provides the steps of the research method. For each activity, the rationale is identified in the last column. The relationships between patterns, issues, solutions and smells is described in Section 3.4.1 below.

\begin{tabular}{|c|c|c|}
\hline No. & Activity & Rational \\
\hline 1 & $\begin{array}{l}\text { Review papers in business modeling and } \\
\text { concurrency research (especially survey papers) }\end{array}$ & \multirow{2}{*}{$\begin{array}{l}\text { Create a framework for } \\
\text { categorizing concurrency } \\
\text { issues and solutions based on } \\
\text { the distributed computing } \\
\text { literature and BPM }\end{array}$} \\
\hline 2 & $\begin{array}{l}\text { Capture patterns, issues, and smells (depending } \\
\text { on paper) in database }\end{array}$ & \\
\hline 3 & $\begin{array}{l}\text { Break issues and solutions out of pattern } \\
\text { descriptions }\end{array}$ & \multirow{6}{*}{$\begin{array}{l}\text { Transform clustered issues } \\
\text { into guidelines using } \\
\text { terminology more familiar to } \\
\text { business process modelers } \\
\text { (vs. computer science) }\end{array}$} \\
\hline 4 & $\begin{array}{l}\text { Derive additional smells from issues (all issues } \\
\text { should in some way be connected to a smell) }\end{array}$ & \\
\hline 5 & $\begin{array}{l}\text { Link records (nodes) based on descriptions and } \\
\text { comparing similar nodes }\end{array}$ & \\
\hline 6 & Categorize smells & \\
\hline 7 & $\begin{array}{l}\text { To create guidelines, generate report for each } \\
\text { data smell by traversing database }\end{array}$ & \\
\hline 8 & $\begin{array}{l}\text { Re-word names and descriptions to improve } \\
\text { understandability and regenerate report }\end{array}$ & \\
\hline
\end{tabular}

Table 4 Research method 


\subsection{Data Acquisition}

As mentioned earlier, data concurrency issues have not been widely studied in BPM research, and there is also relatively little literature on "Smells". However, just as the term "smell" can be applied to "Guidelines for finding problems", there are a lot of other terms that can be used as well. The VIDE Consortium did a systematic survey of the literature up to 2007 on quality defects in model driven software (Rech et al. 2007), and reviewed approximately 950 papers. From this, they created a list of related topics that can be used as search criteria to find more issues:

Anomalies, Anti-guidelines, Anti-patterns, Bug Patterns, Bug Taxonomies, Critic Rules, Defects, Defect Patterns, Design Bugs \& Errors, Error Patterns, Fault Patterns, Flaws, Heuristics, Illnesses, Metric Thresholds, Negative Patterns, Pitfalls, Design Principles, Puzzles / Puzzlers, Design Rules, Code Sins, Styles, Conventions, and Rules (Rech et al. 2007)

The search was also expanded beyond VIDE's. Any research area dealing with reliability involving people and/or data interacting concurrently might contain patterns that could be applied to BPM. Some that come to mind are: Distributed/Concurrent Computing (e.g. Telecommunications \& Real-time Systems), Management Information Systems (MIS), Software Reliability, Software Refactoring, Service Oriented Architectures (SOA), Business Process Re-engineering (BPR), and Human Reliability research.

Finally, literature surveys were focused on, in order to simplify finding a representative sample based on other researchers review criteria, and to make use of the correlation work they had already done. 


\subsubsection{Node Types}

As mentioned earlier, patterns were broken into 3 pieces: Issues, Solutions and the Pattern's Implementation. This allowed a richer set of linkages to be created between them. Smells were them connected to sets of issues, which may connect multiple solutions and patterns, as can be seen in Figure 2 Note Types and Relations"

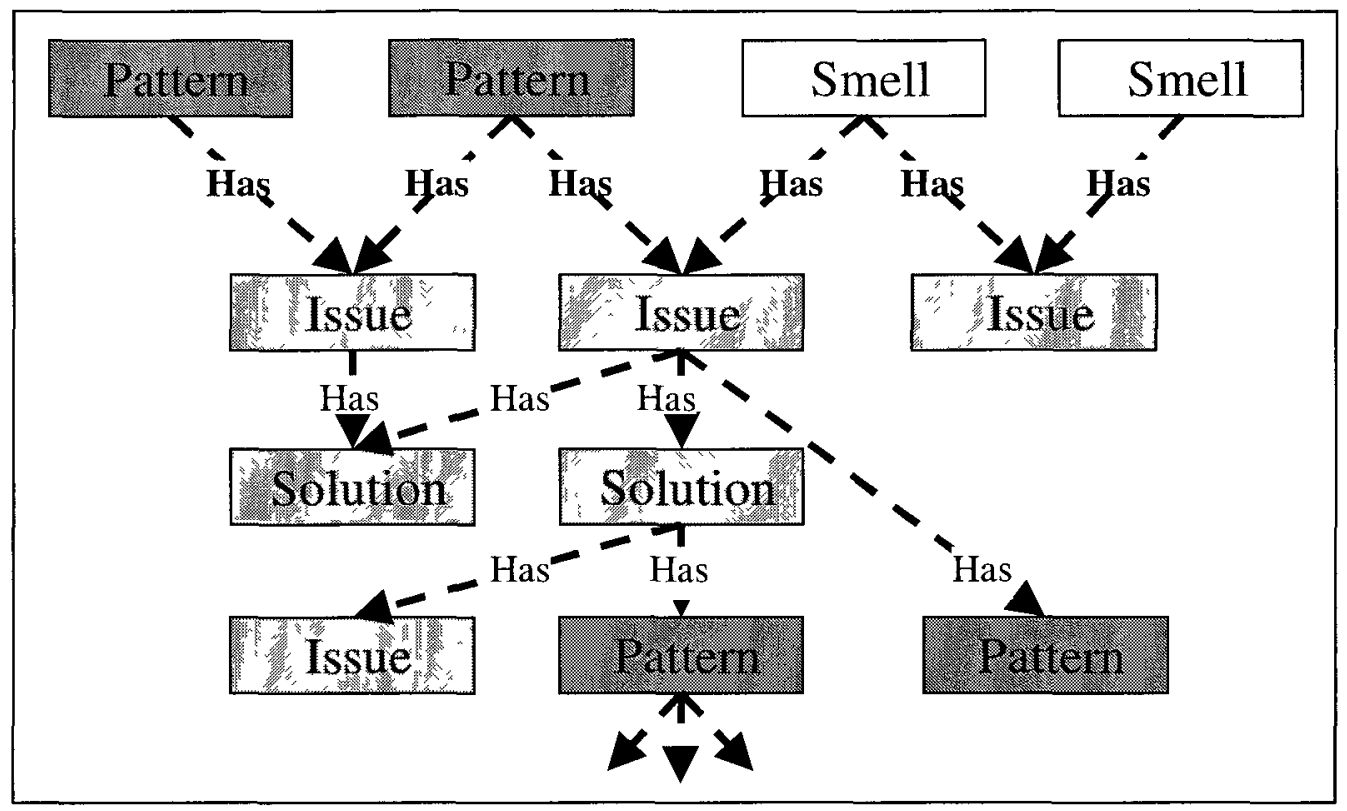

Figure 2 Note Types and Relations

This can be described textually as (a formal entity relationship diagram is provided later):

- A Node can be a Pattern, Smell, Issue, or Solution

- A Pattern has zero or more Issues. A Smell has one or more Issues.

- An Issue Has zero or more Solutions (or solution Patterns), and is Had-by zero or more Patterns or Smells

- A Solution Has zero or more Patterns and Issues 
- Ordinarily a Pattern is made of Issues connected to Solutions, but here a Pattern becomes primarily a reusable algorithm (way of doing things)

- All nodes should be associated with Smells, either directly or indirectly, (otherwise they will never appear in a guideline). Indirectly connected, means that a path exists from an Issue to a Smell, regardless of the direction of the links.

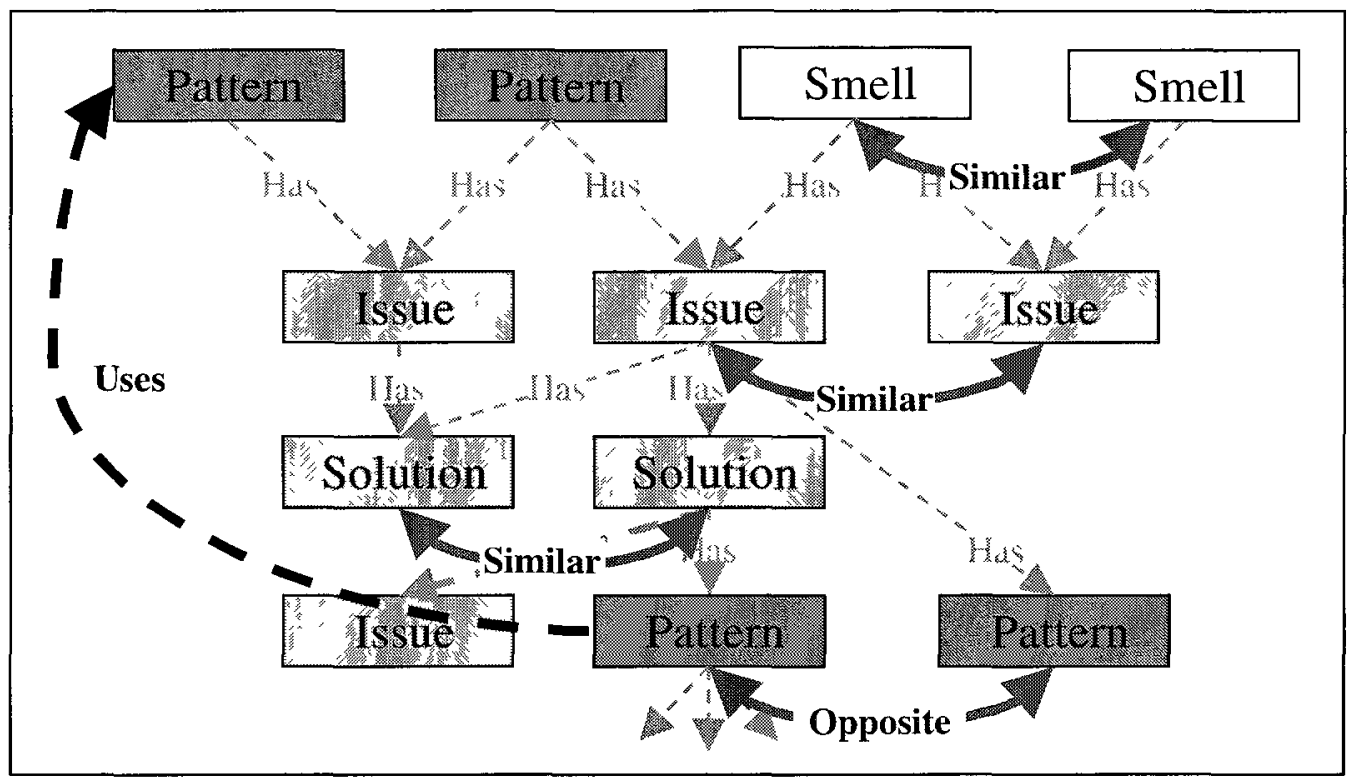

Figure 3 Integration Links

In addition, links can be drawn between nodes of the same type, as is shown in Figure 3 Integration Links".

- A Pattern may Use other Patterns and therefore inherit some of their Issues.

- Patterns, Smells, Issues and Solutions may be similar or opposite to other nodes of the same kind. These links are two-way relationships unlike all the previous types of links which are one-way. 
Figure 4 shows the corresponding entity relationship diagram using Chen's notation (Chen 1976). The double line between the Smell node and its Has relationship means that a Smell must be connected to at least one Issue directly. All the other nodes do not have any mandatory connections, although as mentioned before without a relationship connecting them indirectly to a Smell they will never appear in a guideline.

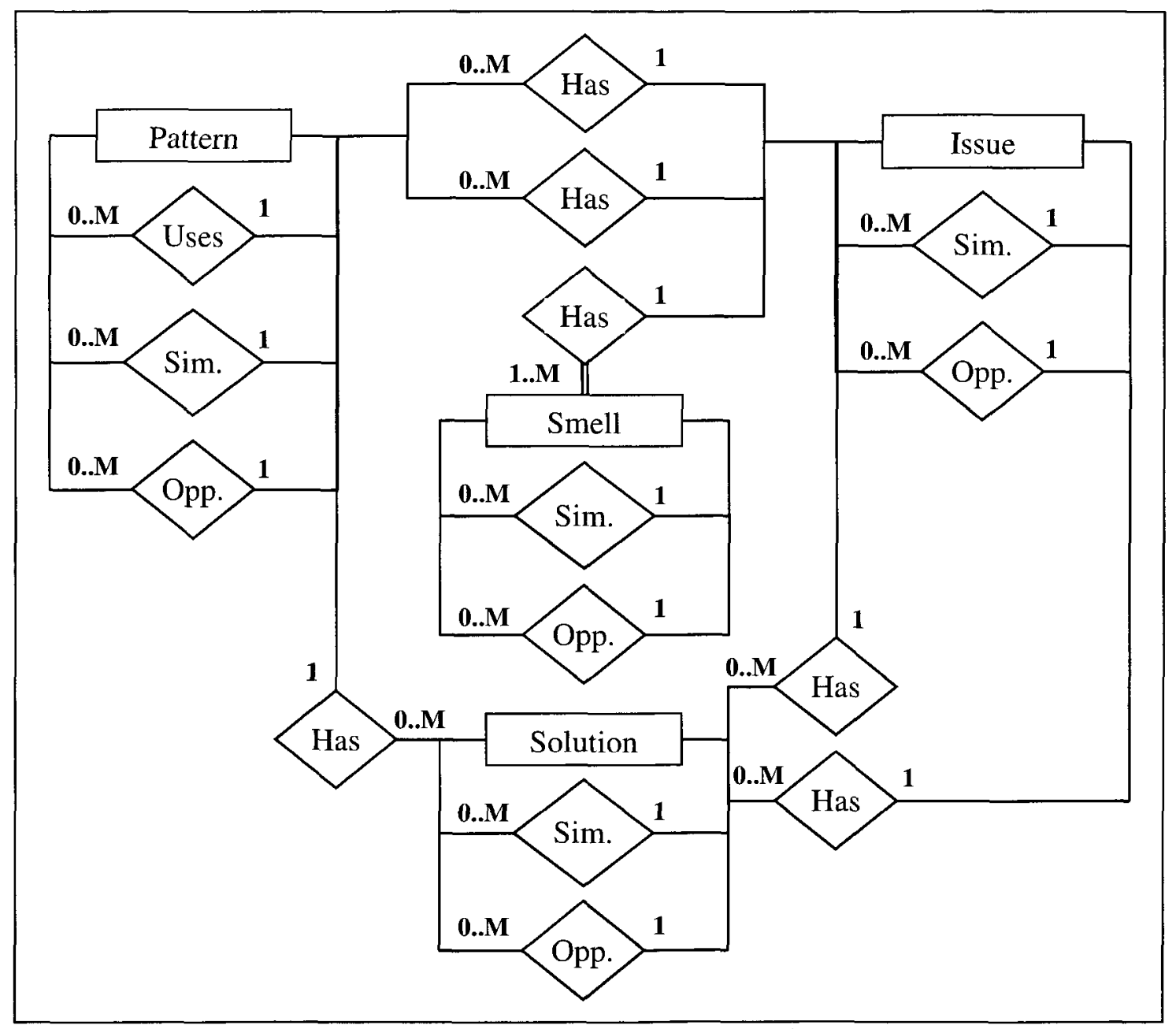

Figure 4. Node Type Entity Relationship Diagram

\subsubsection{Database Structure}

The actual database used to capture the nodes and links is a simple relational database, 
with one table holding the Links, and another the Nodes, as shown in Figure 5. Figure 6 Database Entity Relation Diagram shows the corresponding entity relationship diagram (based on Riccardi 2002). With such a structure, it is possible to create any type of graph, as long as care is taken to keep the links and nodes in sync with each other. This structure allowed importing the data into various tools for editing, graphing, analysis and querying.

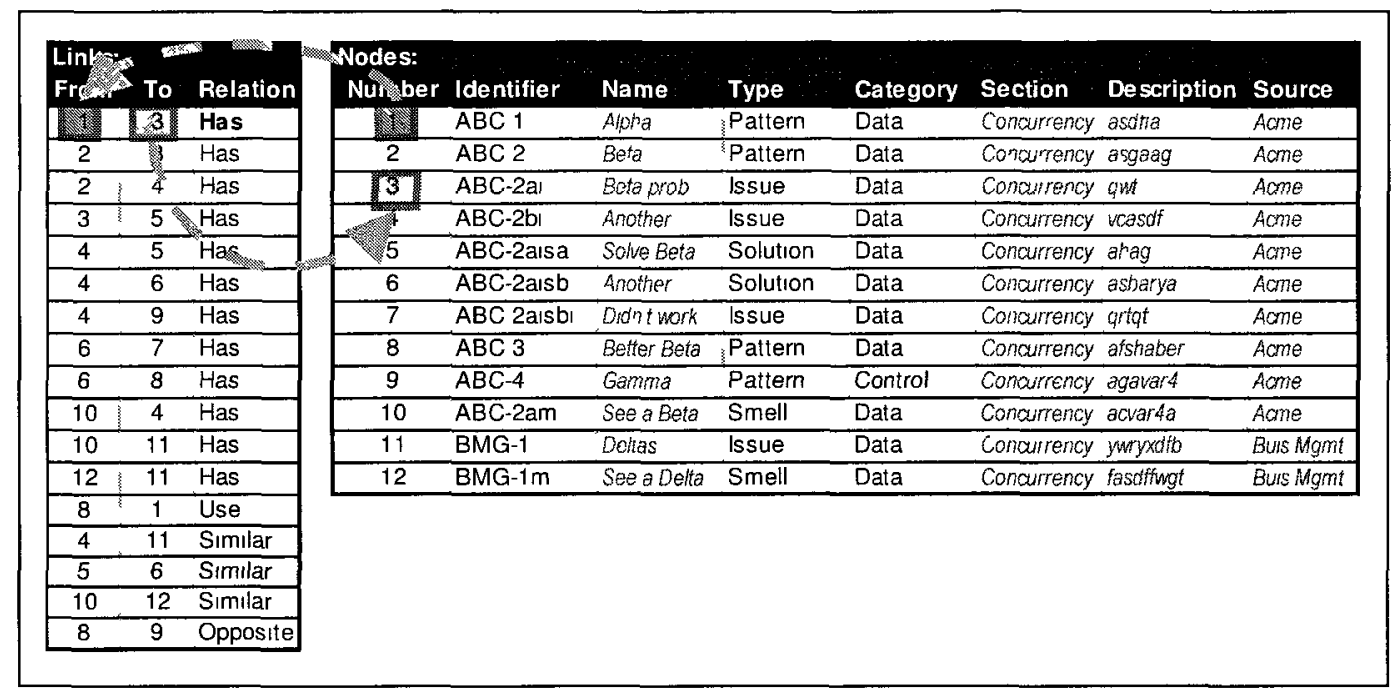

\section{Figure 5 Smell Database Structure}

The report is generated by doing a depth first traversal of the tree rooted at each smell, on a section by section basis. This is discussed in more detail in Section 4.5 "Description Depth".

In Figure 6, Link has double lines to show it is a weak entity which is identified by its To and From relationships. In addition Link must have To and From relationships (double line) while Node does not (single line). That is because a Link must be connected to nodes, while a Node does not need to be connected to any Links. Nodes use a unique 
number as a key. They also have a unique identifier but that is just an implementation detail to be more humanly readable and not an important part of the framework.

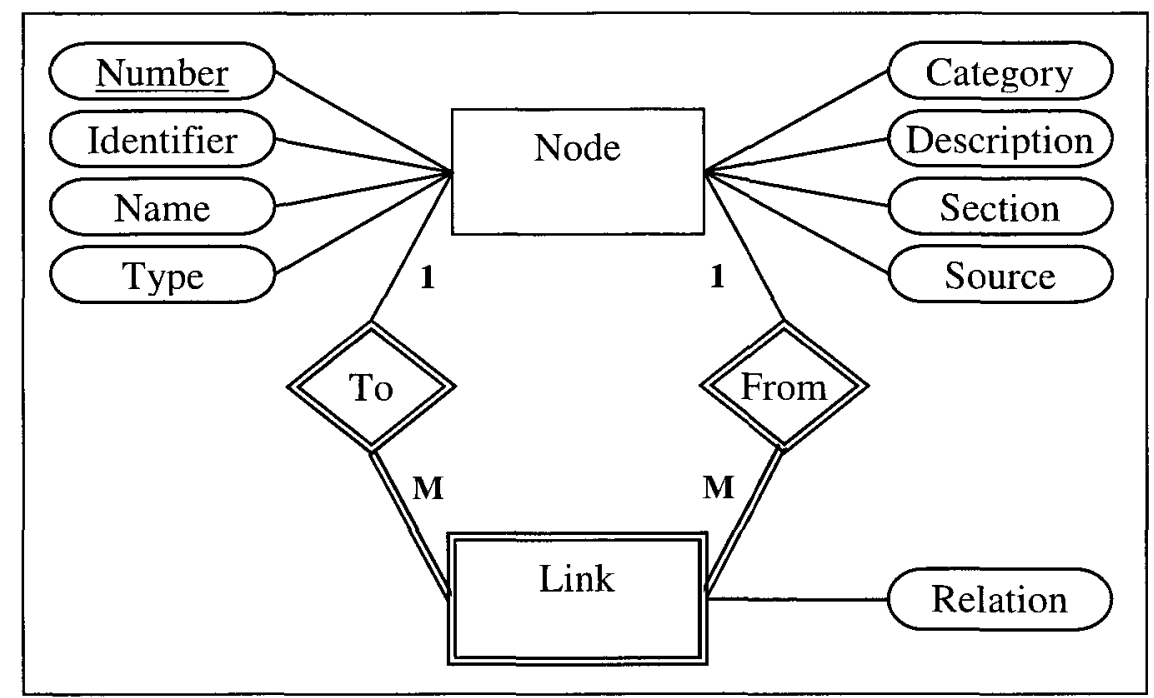

Figure 6 Database Entity Relation Diagram

Nodes contain references back to their sources so the modeler can find detailed information how (or even if) the smell, issue, solution or pattern implementation applies to their context.

When creating the database the information from each paper was captured before it was connected to other sources. When a smell, issue, solution or pattern appears in more than one source then it may be captured as two nodes connected to each other as being similar. If a node truly is a duplicate of another, then it may still make sense to combine them to reduce complexity, but their links to other nodes from their respective sources would still remain. 
For example: if many solution nodes had the same issue about not being supported in a particular language, it may make sense to combine them into a single "Not supported in language XYZ" issue node. The originating solution nodes will still have references back to where this information came from, which the reader could use to find a possible workaround from one (or more) of the related solutions' documents.

Figure 4 showing the relationships between the node types, and Figure 6 showing the database attributes, can be combined together. The node types in Figure 4 are sub-classes of the Node entity in Figure 6, and the Link types in Figure 4 are sub-classed and simplified from the Link entity and To and From relations in Figure 6. They are diagramed separately to make relationships easier to understand. 


\section{RESULTS}

This chapter is organized in six sections. Section 4.1 lists the specific sources used to create the database. Section 4.2 categorizes the pattern information. Section 4.3 discusses an orthogonal break-down into sections. Section 4.4 presents a detailed data analysis Section 4.5 shows how different search depths affect the results, followed by section 4.6 which gives example Smell descriptions and guidelines. Finally, section 4.7 suggests an interactive approach to navigate the relationships between the guidelines.

\subsection{Sources}

Currently, the database contains only a small sampling of the types of papers discussed in "Data Acquisition" (Section 3.4 above). In the guidelines generated from the database (in the appendices) the papers will be referenced using these acronyms:

CBP Concurrent Bug Patterns and How to Test Them, (Farchi et al. 2003)

DAP Data-Flow Anti-patterns: Discovering Data-Flow Errors in Workflows, (Trčka et al. 2009)

PAP Process anti-patterns: How to avoid the common traps of business process modeling, (Koehler \& Vanhatalo, 2007)

PMG Seven Process Modeling Guidelines (7PMG), (Mendling et al. 2010)

WCP Workflow Control-Flow Patterns: A Revised View, (Russell et al. 2006)

WDP Workflow Data Patterns, (Russell et al. 2004a) 
WRP Workflow Resource Patterns, (Russell et al. 2004b)

There are also a few records whose source is listed as "tbd". These are based on the author's experience and supporting sources have not been entered into the database yet. In some cases where there is a source but it has not been fully captured in the database, it is documented in the description.

With the exception of DAP and PMG (which are fairly new) all of these survey papers are heavily referenced in the literature and therefore make a strong foundation for this exercise.

WDP and DAP both comment about the lack of research on data flow problems in Business Process Modeling. With the exception of CBP, this small set of papers may make up the majority of research in our specific research area. However, as discussed previously, there is a lot of related research into both data and concurrency that can be applied, CBP being just an example.

DAP, PMG, WCP, WDP and WCP, all have W.M.P. van der Aalst as one of the coauthors for these papers and can be considered as loosely related as far as content and supporting data.

\subsection{Categories}

The database re-uses the categories from the WorkflowPattern repository (WCP, WDP, and WRP): 
- Control

The control-flow perspective captures aspects related to control-flow dependencies between various tasks (e.g. parallelism, choice, synchronization etc)

- Data

The data perspective deals with the passing of information, scoping of variables, etc.

\section{- Resource}

The resource perspective deals with resource to task allocation, delegation, etc.

The WorkflowPattern repository uses a fourth category as well, Terminations, which is applicable to Data Concurrency issues, but it was organized differently and not analyzed as part of this study. Also, one of the sections, which spanned the Control, Data, and Resource categories, deals specifically with Termination patterns.

\subsection{Sections}

Sections capture themes which may appear in one or more of the categories above. Many of these began as sub-categories from the WorkflowPattern repository but quickly expanded outside their original categories as information from the various sources was analyzed.

- Concurrency: Dealing with parallel execution 
- External: Data interactions with entities external to the BPM. (originally only under Data in WDP)

- Internal: Data interactions internal to the BPM (originally just WDP)

- Robustness: Dealing with fault tolerance or avoidance

- Routing: How data can influence the behaviour BPM. (originally just WDP)

- State: Dealing with condition or mode of being, as in a Finite State Machine (originally just WCP)

- Termination: Dealing with the end of execution, intended or otherwise (originally just WCP)

- Transfer: How data may be transferred (originally just WDP)

- Trigger: External signals which affect control flow (originally just WCP)

- Usability: The comprehensibility of the model (primarily from PMG and PAP)

- Usage: The construction/syntax of the model

- Visibility: How data can be accessed (originally just WDP)

An additional section covered specific Language topics, but was not included in the analysis, because this thesis is intended to be independent of modeling languages, and since languages evolve these issues would have had to be checked for relevancy. 
In WDP (Russell et al. 2004a), the External and Internal "sub-categories" are two halves of a larger sub-category dealing with Data Interactions. This is very closely related to Data Transfer which describes "how" External and Internal transfers operate.

Usability deals primarily with Comprehensibility,. i.e. how easy is the model to understand by the author and others, especially when it comes to ensuring the model does what the author intended. Likewise, Testability, Maintainability, Complexity and other 'ilities could also have been used. Usability is not the perfect name for this section, but is perhaps the simplest.

The Concurrency, Robustness, Usage, (and Language) sections were derived as part of the research for this thesis rather than being re-used from a specific source.

\subsection{Data analysis}

As discussed in section 4.1 above, the database is based on most of the available BPM data concurrency research as well as a few additional sources.

The database contains: 387 Nodes (i.e. records, 157 Data, 150 Control, and 80 Resource) as well as 679 Links: (506 Has, 69 Uses, 101 Similar, and 3 Opposite). It makes sense that it has more links than nodes. Most nodes were created by splitting pattern descriptions apart, and data smells were connected to issues. At a minimum the number of links cannot be much less than the number of nodes. A pattern description may have multiple issues, and implementations, as well as references to other patterns. Finally as part of the contributions of this research, connections were created between related nodes resulting in $75 \%$ more links than nodes. 


\begin{tabular}{|l|r|r|r|r||r|}
\hline & Issue & Pattern & Smell & Solution & Total \\
\hline Control & 65 & 45 & 1 & 39 & 150 \\
\hline Data & $\mathbf{5 6}$ & $\mathbf{4 1}$ & $\mathbf{2 5}$ & $\mathbf{3 5}$ & $\mathbf{1 5 7}$ \\
\hline Resource & 19 & 43 & 0 & 18 & 80 \\
\hline \hline Total & 140 & 129 & 26 & 92 & 387 \\
\hline
\end{tabular}

Table 5 Database statistics

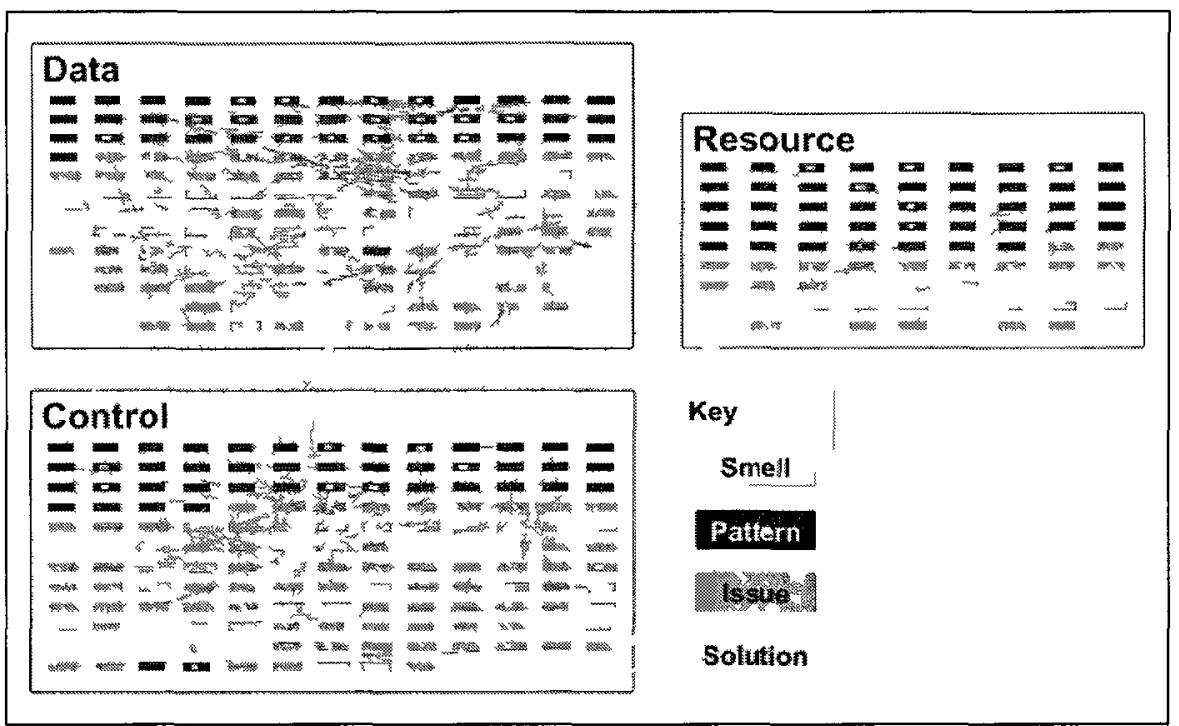

Figure 7 Graphical Representation of Database

\subsection{Description Depth}

The database is essentially a collection of directed graphs Depending on the research source, each graph may be rooted in any type of node As discussed previously, all Data Issues have been connected to at least one Smell, and links have been added between nodes creating new relatıonships when possıble

To create the descriptions of Data related Smells in the form of patlets, each Smell is essent1ally "picked" up and what comes with it become the issues and solutions within its guidelınes However, these connected graphs may contain dozens of nodes, with cyclic links so there has to be a stopping condition or some of the descriptions will be too large 
to be understandable. (Later, in section 4.7, an interactive approach to navigation is suggested to solve this problem)

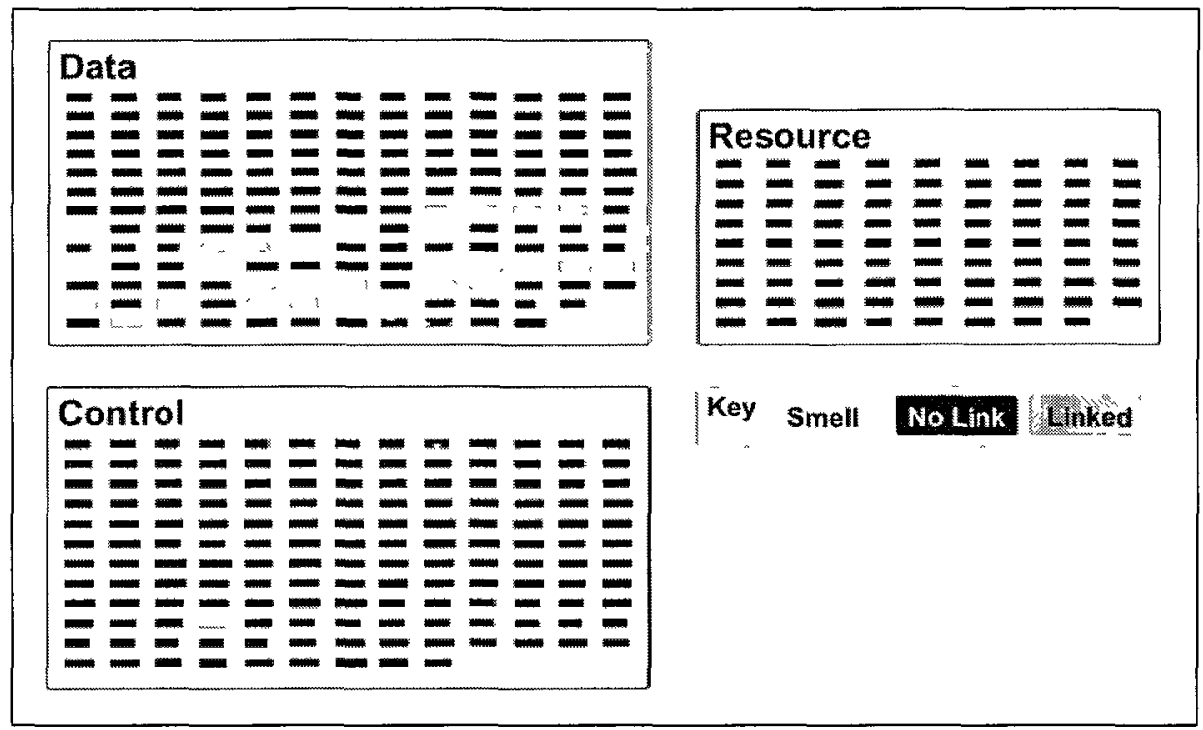

Figure 8 Depth of 1 - Just Smell Nodes

Figure 8 shows a tree depth of one, i.e. just the Smell nodes (other nodes have been darkened to represent that they are not linked). See Appendix A for the list. A depth of two would include the Smells and their directly connected Issues.

\begin{tabular}{|l|r|r|r|r|r|r|r|r|r|r|}
\hline & Issue & Pattern & Smell & Solution & Total \\
\hline Control & 13 & 10 & 1 & 20 & 44 \\
\hline Data & $\mathbf{5 2}$ & $\mathbf{2 8}$ & $\mathbf{2 5}$ & $\mathbf{3 1}$ & $\mathbf{1 3 6}$ & $\mathbf{9 3 \%}$ & $\mathbf{6 8 \%}$ & $\mathbf{1 0 0 \%}$ & $\mathbf{8 9 \%}$ & $\mathbf{8 7 \%}$ \\
\hline Resource & 0 & 0 & 0 & 0 & 0 \\
\hline \hline Total & 65 & 38 & 26 & 51 & 180 & $0 \%$ & $0 \%$ & -- & $0 \%$ & $0 \%$ \\
\hline $46 \%$ & $29 \%$ & $100 \%$ & $55 \%$ & $47 \%$ \\
\hline
\end{tabular}

Table 6 Database statistics for depth of 3

Table 6 shows the quantity and percentage of the total (from Table 5) for each type and category of node that has been "picked" up by traversing a tree to the depth of three. The 
trees now fan out to all the nodes the Smells' Issues are connected to, and covers most of the Data nodes. However, it only goes one level past the Issues. This will reach Patterns and Solutions in the tree, but none of the Uses links which are primarily between Patterns, and therefore the next level down.

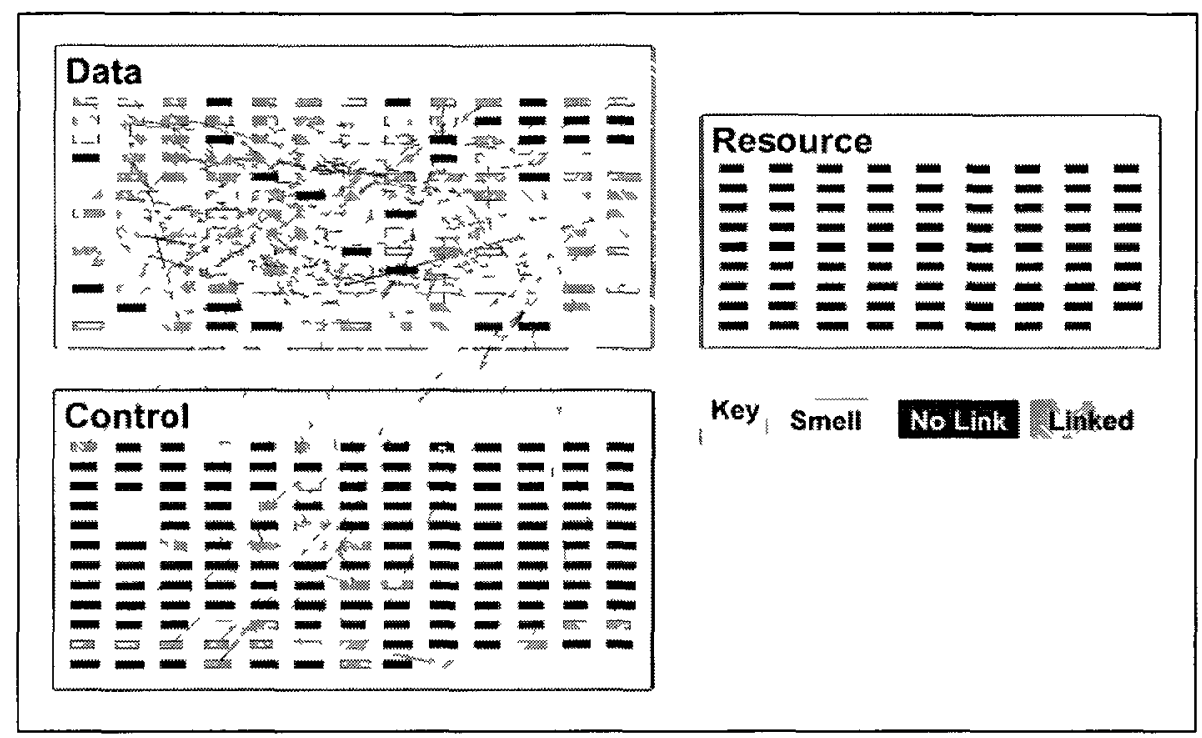

Figure 9 Depth of 3

This is shown graphically in Figure 9. Nodes that have been picked up as part of trees with a depth of three are shown in medium grey. Smell nodes, which are the roots of the trees, are still white.

Figure 10 shows a depth of four which does a much better job of capturing the interconnections. 


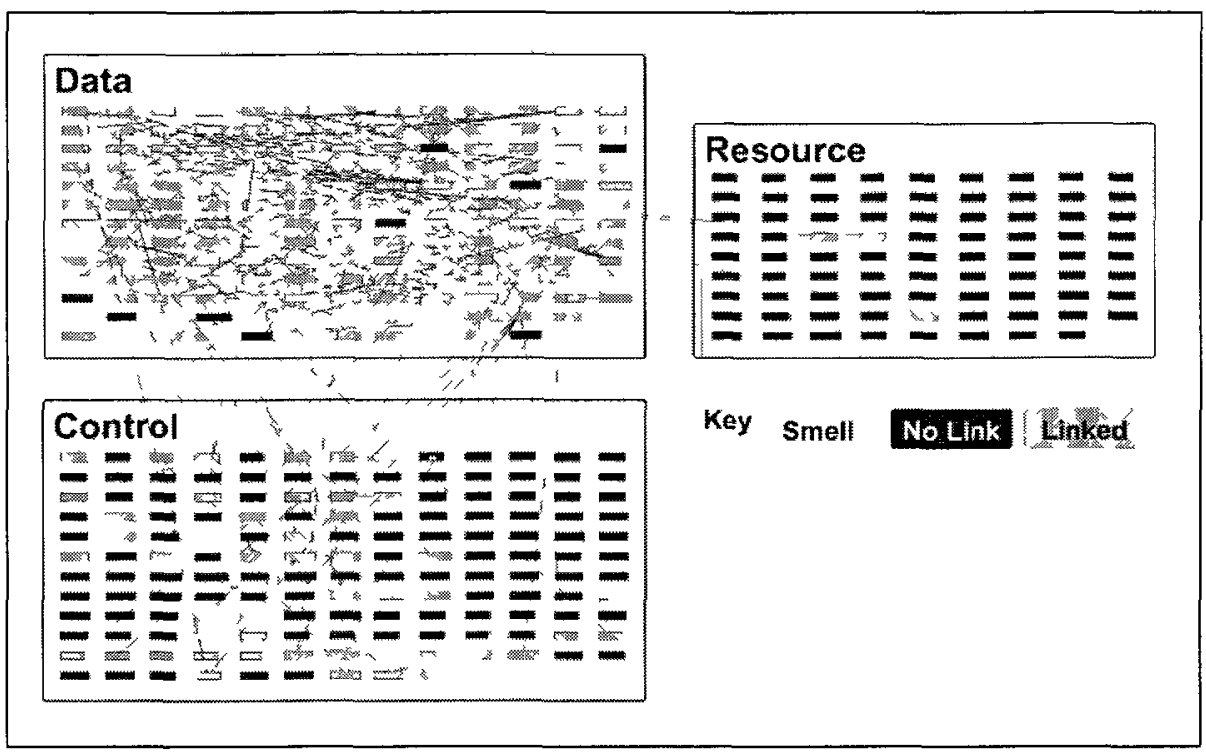

Figure 10 Depth of 4

\begin{tabular}{|c|c|c|c|c|c|c|c|c|c|c|}
\hline & Issue & Pattern & Smell & Solution & Total & Issue & Pattern & Smell & Solution & Total \\
\hline $\mathrm{Co}$ & 26 & 24 & 1 & 26 & 77 & $40 \%$ & $53 \%$ & $100 \%$ & $67 \%$ & $51 \%$ \\
\hline Data & 54 & 39 & 25 & 34 & 152 & $96 \%$ & $95 \%$ & $100 \%$ & $97 \%$ & $97 \%$ \\
\hline rce & 1 & 2 & 0 & 1 & 4 & $5 \%$ & $5 \%$ & -- & $6 \%$ & $5 \%$ \\
\hline Total & 81 & 65 & 26 & 61 & 233 & $58 \%$ & $50 \%$ & $100 \%$ & $66 \%$ & $60 \%$ \\
\hline
\end{tabular}

Table 7 Statistics for depth of 4

Figure 11 contınues to a depth of 5 and captures the last of Data's Issues and Solutions.

Th1s also further 1ncreases the size of each Smell's tree of related nodes, making them confusing and less useful.

Note, there is st1ll one Data issue missing: "Data-based trigger not directly supported?" (WDP-39). It is connected to the "External" smell "Sending data outside of the Workflow" (WDP-15), indirectly as a form of delivery acknowledgement, but is at a depth of six. Strictly speaking it is one of the "Language" specific issues which were 
excluded from the analysis, however since it has a workaround, it was placed in the "Routıng" section instead It also not a data concurrency issue

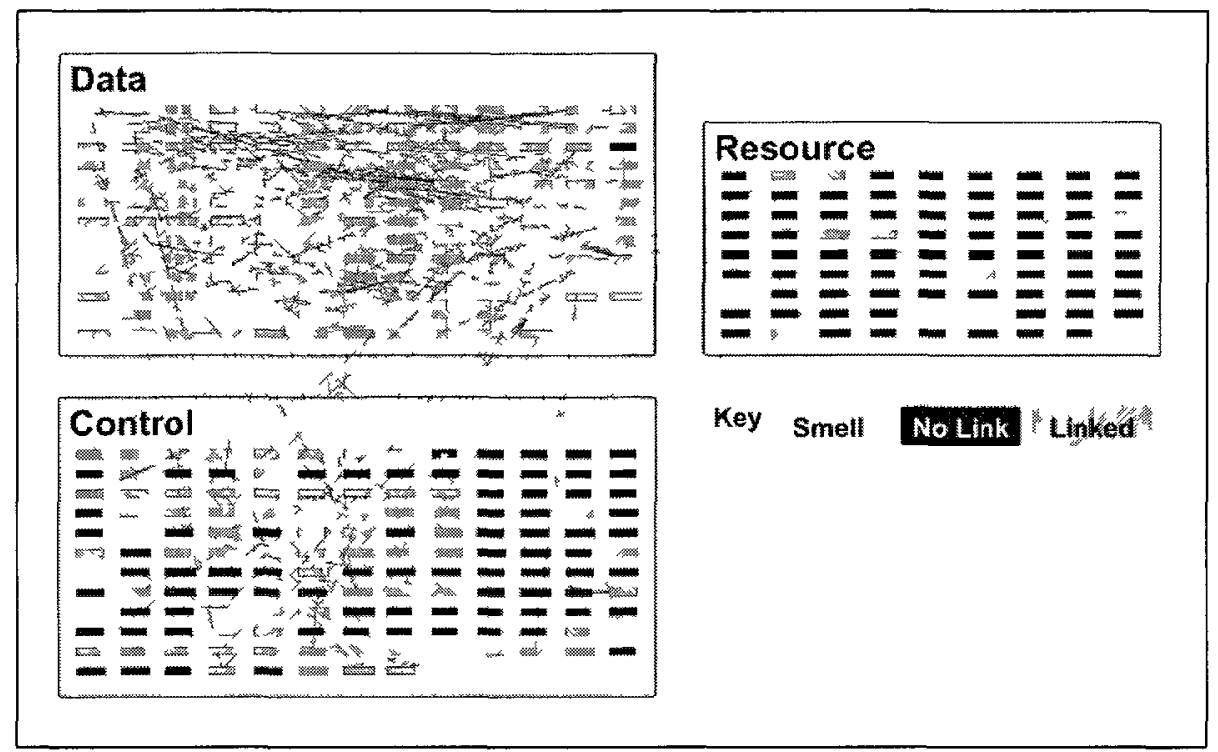

Figure 11 Depth of 5

\begin{tabular}{|l|r|r|r|r|r|r|r|r|r||r|}
\hline & Issue & Pattern & Smell & Solution & Total & Issue & Pattern & Smell & Solution & Total \\
\hline Control & 39 & 36 & 1 & 32 & 108 & $60 \%$ & $80 \%$ & $100 \%$ & $82 \%$ & $\mathbf{7 2 \%}$ \\
\hline Data & $\mathbf{5 5}$ & $\mathbf{4 1}$ & $\mathbf{2 5}$ & $\mathbf{3 5}$ & $\mathbf{1 5 6}$ & $\mathbf{9 8 \%}$ & $\mathbf{1 0 0 \%}$ & $\mathbf{1 0 0 \%}$ & $\mathbf{1 0 0 \%}$ & $\mathbf{9 9 \%}$ \\
\hline Resource & 3 & 6 & 0 & 3 & 12 & $16 \%$ & $14 \%$ & - & $17 \%$ & $15 \%$ \\
\hline \hline Total & 97 & 83 & 26 & 70 & 276 & $69 \%$ & $64 \%$ & $100 \%$ & $76 \%$ & $71 \%$ \\
\hline
\end{tabular}

Table 8 Statistics for tree depth of 5

Nodes which are similar to each other can be considered to share all the same links, but since they are stored as a graph, shared links between simılar nodes are treated as being one link further away The amount of information can be increased while reducing the depth by flattening these "similarity" trees and treatıng them all as being at an equivalent level Table 9 and Table 10 below, show the new statistics 


\begin{tabular}{|c|c|c|c|c|c|c|c|c|c|c|}
\hline & Issue & Pattern & Smell & Solution & Total & Issue & Pattern & Smell & Solution & Total \\
\hline Control & 17 & 31 & 1 & 23 & 72 & $26 \%$ & $69 \%$ & $100 \%$ & $59 \%$ & $48 \%$ \\
\hline Data & 252 & 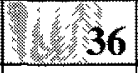 & 25 & 33 & 146 & $93 \%$ & $88 \%$ & $100 \%$ & $94 \%$ & $93 \%$ \\
\hline Resource & 1 & 3 & 0 & 1 & 5 & $5 \%$ & $7 \%$ & -- & $6 \%$ & $6 \%$ \\
\hline Total & 70 & 70 & 26 & 57 & 223 & $50 \%$ & $54 \%$ & $100 \%$ & $62 \%$ & $58 \%$ \\
\hline
\end{tabular}

Table 9 Statistics for depth of 3 with similar nodes promoted.

\begin{tabular}{|c|c|c|c|c|c|c|c|c|c|c|}
\hline & Issue & Pattern & Smell & Solution & Total & Issue & Pattern & Smell & Solution & Total \\
\hline Control & 45 & 39 & 1 & 29 & 114 & $69 \%$ & $87 \%$ & $100 \%$ & $74 \%$ & $76 \%$ \\
\hline Data & 54 & 41 & 25 & 34 & 154 & $96 \%$ & $100 \%$ & $100 \%$ & $97 \%$ & $98 \%$ \\
\hline Resource & 3 & 9 & 0 & 4 & 16 & $16 \%$ & $21 \%$ & - & $22 \%$ & $20 \%$ \\
\hline Total & 102 & 89 & 26 & 67 & 284 & $73 \%$ & $69 \%$ & $100 \%$ & $73 \%$ & $73 \%$ \\
\hline
\end{tabular}

Table 10 Statistics for depth of $\mathbf{4}$ with similar nodes promoted

Data node coverage increased noticeably $(+6 \%)$ for a depth of 3 , and slightly $(+1 \%)$ for a depth of 4 . However, the overall coverage of a tree with a depth of 3 and similar nodes promoted is very close to the overall coverage of a tree with the depth of 4 without promotion (58\% vs. 60\%). The over-all coverage of a tree with a depth of 4 with promotion is actually slightly better than the depth of 5 without promotion (73\% vs. $71 \%$ ), making this a notable improvement.

\subsection{Example Smell Description}

This section shows two examples, the first demonstrating expanding a description to a depth of 3 , and the second to a depth of 4 . Both promote similar nodes to be treated as siblings. 


\subsubsection{Example smell description, depth of 3}

In Figure 12 below, a depth of 1 only includes the Smell "Making changes...", while a depth of 2 captures the Issue "Passing data...". It will also promote all similar issues, like "Unknowable outcome" regardless the length of similar links.

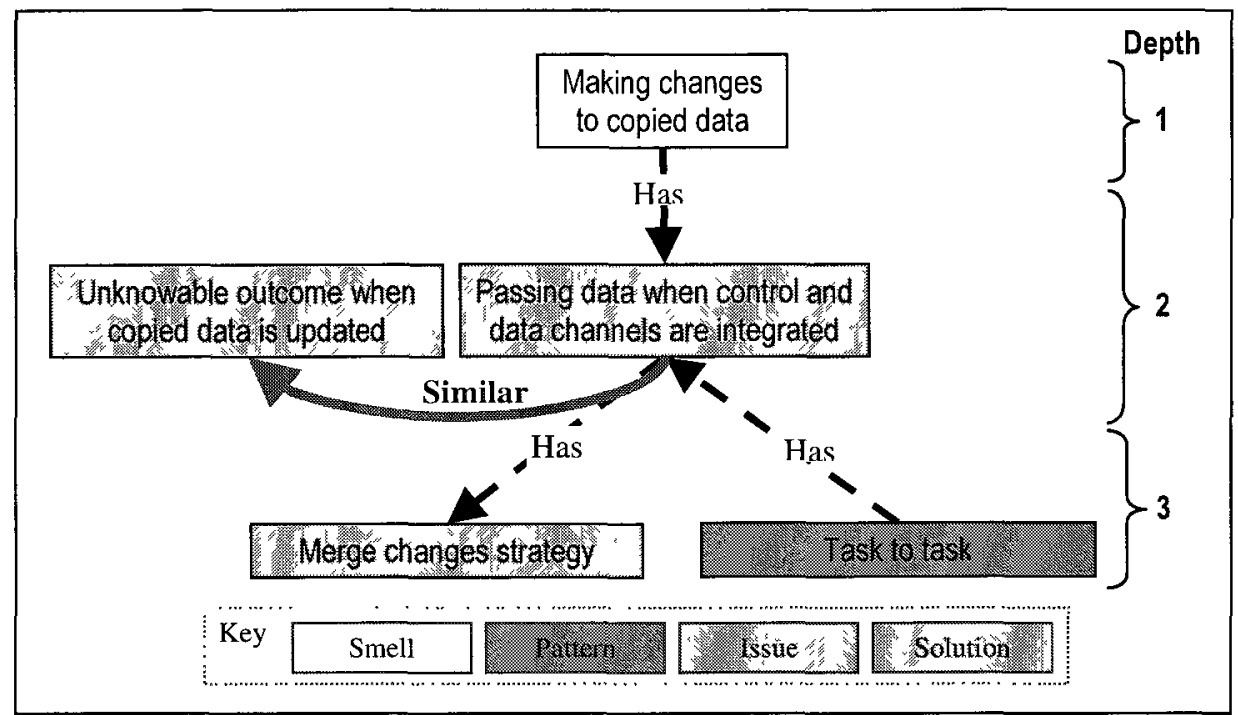

Figure 12 Example Tree - Depth 3

In this example there is only one similar link and "Unknowable outcome..." also happens to be directly connected to "Making changes..." (link not shown in diagram). When this happens, "Unknowable outcome..." should not show up in the Smell's listing twice.

When expanding the depth of the description, the direction of the links is not important. In this example, at a depth of 3 , only the links connected to "Passing data..." are followed. The Issue has the Solution "Merge changes..." and has a reverse connection to the Pattern "Task to Task". A reverse connection means that it is the Pattern "Task to Task" that has the Issue "Passing data..." 
In the example smell description listing below, as in the appendices, smells are divided into sections. Each individual smell, pattern, issue or solution's name is followed by its category and its source. In the description for "Making changes...", you can see it is in the Concurrency section, a member of the Data category, Concurrency section (of course), and its source is WDP (Russell et al. 2004a) pattern 29.

\section{Concurrency:}

\section{Making changes to copied data Data Concurrency WDP-29}

Whenever data is shared by making copies of it, then it is very easy for the copies to cease being the same.

\section{Consider Issue:}

Passing data when control flow and data Data Internal WDP-9 travel together

(also known as: Passing data when control and data channels are integrated)

If the data for an activity and control of the activity are integrated, and if the data was copied to parallel paths, then it is possible that two (or more) different copies of the data will arrive at the same location. For example, while building a house, copies of the plans are passed along to each new trade as they take over an area - along with notes, changes, deletions and possibly mistakes. Eventually someone will receive two copies of the plans which are no longer the same. 


\section{Consider Solution:}

Merge changes strategy

Data Transfer

WDP-29

\section{Related to Pattern:}

Task to task

Data Internal WDP-9

Unknowable outcome when copied data is Data Transfer WDP-29 updated

[...description...]

In the description listing, using "Has a" or "Had by" to express relations is not easy to understand, so "Has" links become "Consider" and reverse links ("Had by") becomes "Related to". The "similar" links have disappeared because they were promoted. So, in this example, you could read it as: If a practitioner sees a model where changes are being made to copied data (smell), then they should look for problems with passing data when control flow and data travel together (issue) and then consider using a merge changes strategy (solution) to fix it. They could also refer to the "Task to Task" pattern for more information.

You may notice that the smell "Making changes..." and solution "Merge change..." come from a different WDP source pattern than the issue "Passing data..." and pattern "Task to Task". This relationship may not be obvious from the original source. This is even less likely if they came from different papers. The complete list (to date) of smell descriptions, to a depth of 3, can be found in Appendix B. 


\subsubsection{Example smell description, depth of 4}

At the depth of 4, "uses" relationships finally appear. Unlike "has" relationships where in the textual description the word is replaced completely, "uses" is less difficult to understand, so "used by" is used to represent the reverse relationship.

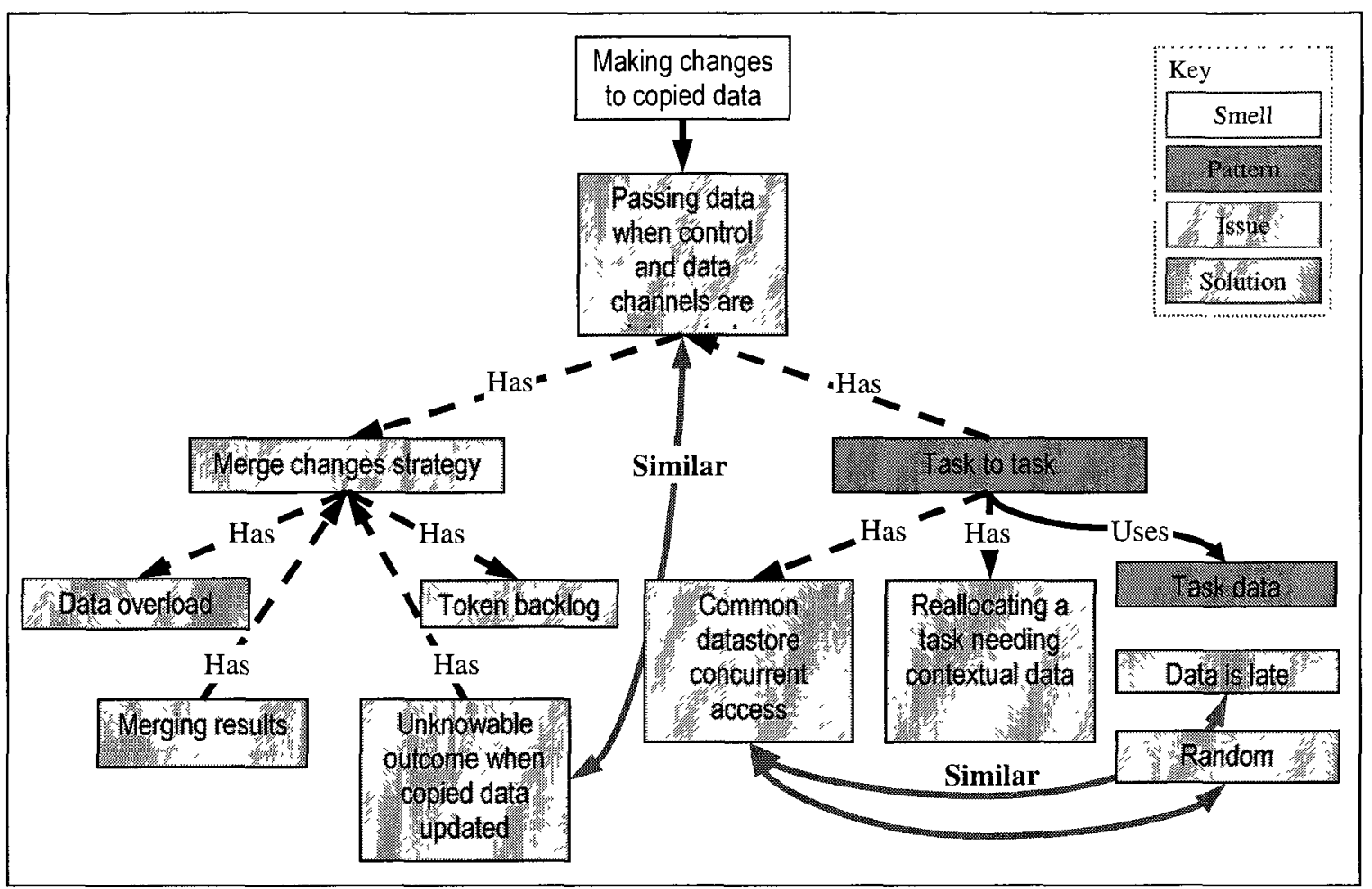

Figure 13 Example Tree - Depth 4

In the example, the issue "Unknowable outcome" appears for the second time, this time directly linked (in reverse) to the solution "Merge changes". Since it is in a different part of the description it needs to be left there. However, if it was at a higher level and was going to be expanded for the second time, the message "PLEASE SEE earlier description" is included rather than duplicating everything. This is only done per section so readers do not have to search too far in the document. 


\section{Concurrency:}

\section{Making changes to copied data Data Concurrency WDP-29}

[...description...]

Consider Issue:

Passing data when control flow and data Data Internal WDP-9 travel together

[...description...].

Consider Solution:

Merge changes strategy

Data Transfer WDP-29

Consider Issue:

Data overload

Data Internal WDP-11

Token backlog

Data Robustness tbd

Related to Issue:

Unknowable outcome when

Data Transfer

WDP-29

copied data is updated

Merging results

Data Internal WDP-12

\section{Related to Pattern:}

Task to task

Data Internal WDP-9

Consider Issue:

Common datastore concurrent

Data Concurrency WDP-5 access

Reallocating a task needing contextual data

Data Concurrency WRP-30 
(also known as: Stateful Reallocation Data Concurrency)

Random problems $\quad$ Control Concurrency WCP-1

Data is late Data Internal WDP-9

Uses Pattern:

Task data

Data Visibility WDP-1

Unknowable outcome when copied data is Data Transfer WDP-29 updated

[...description...]

Note that the source of "Token backlog" is "tbd". The issue comes from Börger et al. (2008) which has not been fully captured in the database. This issue's description can be found in the Robustness section under "Using the token design pattern" (appendix section B.6.1).

In this example, you could expand the description for the "Making changes to copied data" smell as: If you use the merge change strategy solution, then you should be aware that it has issues with data overload and token backlog. This is also a solution for issues with unknowable outcomes when copied data is updated and merging results. Similarly, if you choose to consider the "Task to task" pattern, then you should know that it has several issues as well to consider and it in turn uses the "Task data" pattern.

\subsection{Navigation}

Description tree depths of four (with similar nodes promoted), do an acceptable job of covering the data concurrency guidelines. However, while a tree of depth 4 has approximately only $25 \%$ more nodes than a tree of depth 3 (see Table 9 and Table 10 
above) these nodes are repeated often because of how tightly interlinked the nodes are (see Section 4.4). Promoting similar nodes further increases the number of children for each node. All together this can make it easy to lose track of where you are in the guidelines. To illustrate this, the last smell in appendix B: B.12.1 "Reusing names for data" takes over two pages as a guideline when expanded to the depth of three. When expanded to a depth of four it takes six pages. Breadcrumbs in the appendices headers help the reader keep track of which smell they are in, but it may still be hard for the reader to remember how the nodes on the fifth page are related to the nodes on the second.

A better way to handle this is with an interactive interface, not possible in a printed document. There are many choices of data browsers, both textual and graphical. For purposes of an example a simple wiki interface was created. Wikis have many advantages for sharing data on the web, and allowing other researchers to add and review content (Weiss et al. 2007b).

In Figure 14 below, a wiki based on Tiddlywiki (specifically Shulman 2009) was created using a similar report generator to the one that created the appendices at the end of the thesis.

The figure shows the same smell guideline as discussed section 4.6.1, but only with only selected branches expanded. The top of the complete list of smells is shown as well. While not shown due to limitations on the size of the printed page, it is possible to drill as deep as desired, including following loops. While this can easily produce too much 
information it is easy to see where you are in the tree, and to collapse parts of the tree that are not of immediate interest. It is also possible to have multiple browsers and use different search criteria to create different views of the guidelines.

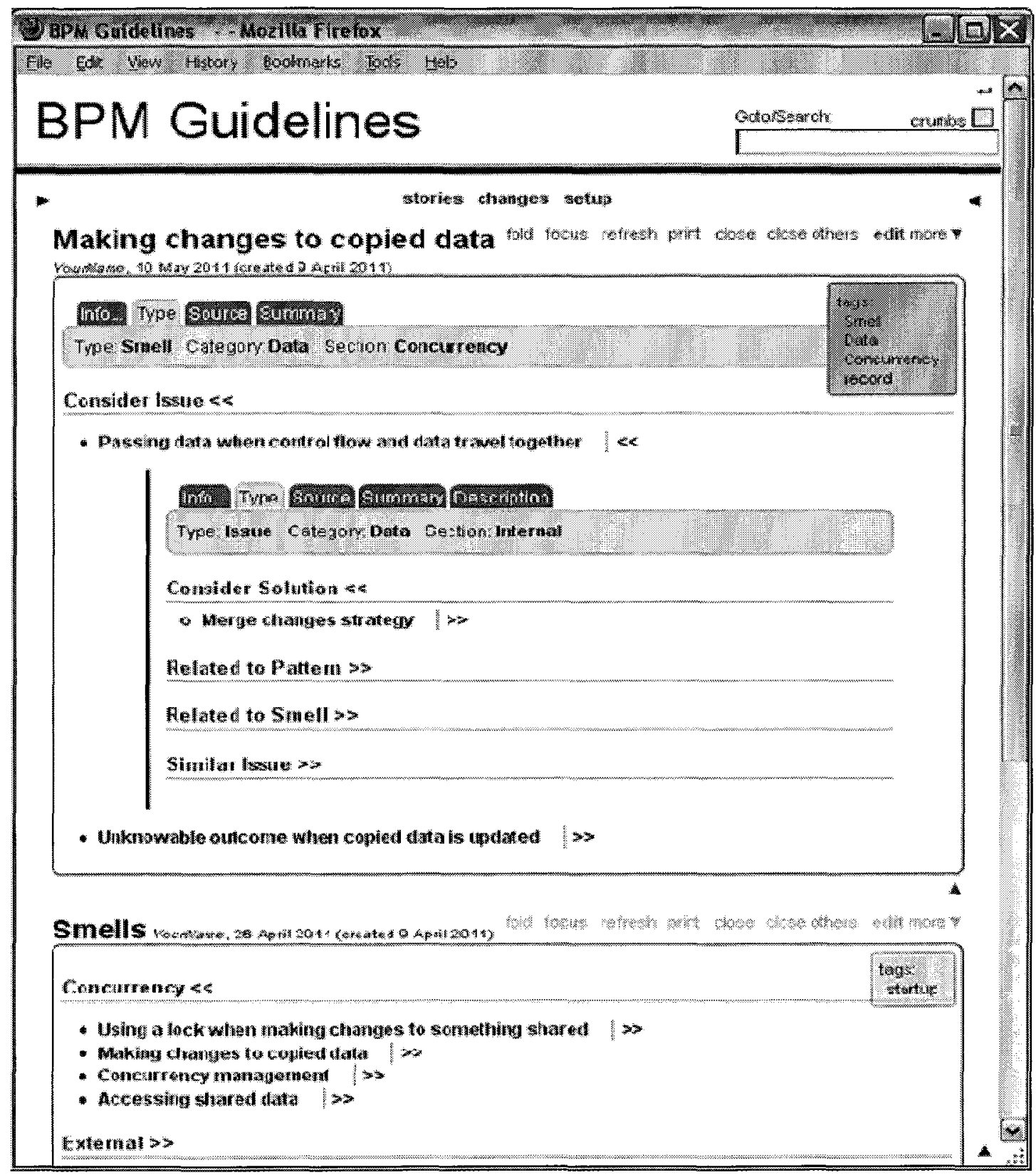

Figure 14 Interactive Guidelines Example 
This was just a simple example, and is left as a suggestion for future research with regards to its effectiveness. 


\section{CONCLUSIONS, LIMITATIONS, AND FUTURE RESEARCH}

\subsection{Conclusions}

Business processes suffer from the same problems as distributed computer systems, even when the work is being done by humans. While there is a great deal of literature on concurrency issues in distributed systems, parallelism and related disciplines in computer science, research in the area of business process modeling is still young and focuses primarily on control flow problems.

The objective of this research was to move modeling knowledge from computer science to the business domain and thus make it available to business process modelers. As there is a gap in the business process modeling research on data concurrency, the thesis specifically focuses on creating guidelines for where to look in models for data concurrency problems, what those problems may be, and possible solutions.

\subsection{Key findings}

1 Patterns are typically organized by the types of solutions they provide and then refer to the contexts to which they may be applied.

a. Reorganizing patterns into smells provided guidelines that showed the reader how to recognize situations which may hide errors, and then lists what the problems may be, how to solve them, and what other issues and problems may present. This is a novel way to view information about data concurrency problems. 
b. The process of codifying the data concurrency knowledge broke the patterns and other knowledge from different sources into their component parts and reconnected the related parts again. While previous research documented related patterns, this process synthesized a richer coupling by combining others' research. This is a generic process that can be applied to other domains, and similarly help extract knowledge from patterns and principles (guidelines) documented in the literature.

2 Literature on data concurrency issues in modeling is typically written in terms not familiar to business people. The language of computer science, with regards to data concurrency, was adapted to language more familiar to the business analysts who design business processes. These proof-of-concept guidelines will help BPM practitioners improve the quality of their models, by being able to make use of knowledge about data concurrency problems, well known in the realm of computer science, of which they were previously unaware.

\subsection{Limitations}

The study has three limitations:

1 The database is incomplete. Only seven out of over a hundred papers and books containing collections of data concurrency problems have been captured in the database.

2 As the current database is small, the framework will need to evolve in order to scale with the complexity of categorizing larger numbers of patterns and being able to use the resulting multitude of guidelines. 
3 Categorization was done manually by a single researcher.

$4 \quad$ Usefulness of guidelines has not been validated with domain experts.

\subsection{Opportunities for future research}

The current research opens many possibilities for future research:

1 Expand the BPM pattern database with additional concurrency patterns

a. Especially in control flow and resource management

b. It would be very interesting to extend this into related performance, fault tolerance and security areas. (Bonabeau 2002)

2 Validate guidelines with subject matter experts to discover if the guidelines are useful and understandable. An "Expert panel" approach is commonly used in Medicine to validate symptom diagnoses, and only requires a small number of experts. Goldenberg et al. (2001) provide an example of it applied to business research.

3 A dynamic interface to navigate the Smells, Issues, and Solutions would make it much easier for readers understand the interrelations than the static listings in the appendices below. A database browser or a custom Wiki would be immensely helpful (Weiss et al. 2007b).

4 Experiment with categorization algorithms to see if there is a more effective grouping.

a. Different types of links may be considered to be of different value

b. Current manual groupings carry relationship information as well 
c. Using coupling and cohesion metrics to form the groups (which may include groups within groups) based on connection weights may be better than the technique of simply breaking links to form groups.

$5 \quad$ Automated analysis may be more effective.

a. Would require all the patterns, issues, guidelines, etc. from the various sources be translated into a consistent formal syntax first.

b. Keyword analysis may be useful but even papers by the same author do not always use words consistently.

6 The list of data concurrency problems could also be used by BPM tool designers to improve model rule checkers.

7 Apply framework to other pattern domains. 
A.1 Concurrency:

A.1.1 Using a lock when making changes to something shared

\section{REFERENCES}

Alexander, C. (1979). The Timeless Way of Building. Oxford University Press, Oxford.

Alexander, C., Ishikawa, S., Silverstein, M., Jacobson, M., Fiksdahl-King, 1. and Angel, S. (1977). A Pattern Language: Towns, Buildings, Construction. Oxford University Press, Oxford.

Amyot, D., Weiss, M., and Logrippo L. (2005), Generation of test purposes from Use Case Maps, Computer Networks, 49(5), 643-660.

Barland, I., Greiner, J., \& Vardi, M. (2005) Concurrent Processes: Basic Issues Connexions. 6 Oct. 2005 http://cnx.org/content/m12312/1.16/

Becker J., Rosemann M., von Uthmann C. (2000), Guidelines of Business Process Modeling, Business Process Management Lecture Notes in Computer Science, 2000, Volume 1806/2000, pp 241-262, DOI: 10.1007/3-540-45594-9_3

Börger, E.,Thalheim, B. (2008) A method for verifiable and validatable business process modeling, Advances in Software Engineering, volume 5316 of LNCS, pp 59115. Springer-Verlag, 2008.

Börger, E., Sörensen, O.,Thalheim, B. (2009) On defining the behavior of OR-joins in business process models, Journal of Universal Computer Science, vol. 14, no. 1, 2009, pp 3-32

Bonabeau, Eric. (2002), Predicting the Unpredictable. Harvard Business Review, March 2002, pp. 109-116.

Bouhours C., Leblanc H., Percebois C., (2009), Bad smells in design and design patterns, Journal of Object Technology, vol. 8, no. 3, May-June 2009, pp. 43-63

Braem M., Joncheere N., Vanderperren W., Van Der Straeten R., Jonckers V., (2006), Guiding Service Composition in a Visual Service Creation Environment, Web Services, 2006. ECOWS '06. 4th European Conference, Zurich , Dec. 2006 , pp 13 - 22, DOI 10.1109/ECOWS.2006.23

Brown, W.J.; Malveau R.C., McCormick H.W., Mowbray T.J., Hudson T. (ed) (1998). AntiPatterns: Refactoring Software, Architectures, and Projects in Crisis. John Wiley \& Sons, ltd. ISBN 0-471-19713-0. 
A.1 Concurrency:

A.1.1 Using a lock when making changes to something shared

Chen P., (1976). "The Entity-Relationship Model - Toward a Unified View of Data". ACM Transactions on Database Systems, March 1976, 1 (1): 9-36.

doi:10.1145/320434.320440.

Chowdhary, P., Bhaskaran, K., Caswell, N., Chang, H., Chao, T., Chen, S., et al. (2006). Model driven development for business performance management. IBM Systems Journal, 45(3), pp 587-605.

Chung, W. W. C. \& Pak, J. J. F. (2006). A case study: using UML to develop a knowledge-based system for supporting business systems in a small financial institute, International Journal of Computer Integrated Manufacturing,19:1, pp 59-68

Cunningham W. and Beck K. (1987) Using pattern languages for object-oriented programs. In proceedings of OOPSLA'87, Orlando, Florida, 1987.

Cunningham, W. (2005), Patlet, Portland Pattern Repository's WikiWikiWeb [Last edited Aug.13, 2005] http://www.c2.com/cgi/wiki?PatLet

Curtis, B., Kellner, M.I. and Over, J. (1992), ‘Process modeling', Communication of the ACM, Vol. 35 No. 9, pp. 75-90.

Davies I., Green P., Rosemann M., Indulska M., Gallo S. (2006), How do practitioners use conceptual modeling in practice?, Data \& Knowledge Engineering 58 (3) (2006) $358-380$

de Castro, V., Marcos, E. and López Sanz, M. (2006). A model driven method for service composition modelling: a case study, Int. J. Web Engineering and Technology, 2(4)335-353.

Dijkman, R.M., Dumas, M., Ouyang, C., (2007), Formal semantics and automated analysis of BPMN process models. Technical Report Preprint 5969, Queensland University of Technology, 2007

Eriksson, H.-E. \& Penker, M. (2000), Business modeling with UML: business patterns at work. New York: John Wiley \& Sons.

Farchi E ,Nir Y , Ur S, (2003), Concurrent Bug Patterns and How to Test Them, Proceedings of the 17th International Symposium on Parallel and Distributed Processing, p.286.2, April 22-26, 2003 
A.1 Concurrency:

A.1.1 Using a lock when making changes to something shared

Fishwick, P.A. (2007), Handbook of Dynamic System Modelling. Chapman \& Hall/CRC: Boca Raton. 1-2.

Fowler M. and Beck K. (1999) Bad Smells in Code, in Refactoring: Improving the Design of Existing Code, Addison-Wesley 1999

Gamma, E.; Helm R., Johnson R., Vlissides J (1995). Design Patterns: Elements of Reusable Object-Oriented Software. Addison-Wesley. ISBN 0-201-63361-2.

Goldenberg J., Lehmann D.R., and Mazursky D. (2001), The Idea Itself and the Circumstances of Its Emergence as Predictors of New Product Success, Management Science, Vol. 47, No. 1, Design and Development. (Jan., 2001), pp. 6984.

Gottschalk, F., Van der Aalst, W.M.P., Jansen-Vullers, M.H., Verbeek, H.M.V., (2006), Protos2CPN: Using Colored Petri Nets for Configuring and Testing Business Processes. Workshop and Tutorial on Practical Use of Coloured Petri Nets and the CPN Tools, Aarhus, Denmark, October 2006. Published online at: http://www.daimi.au.dk/CPnets/workshop06/.

Guceglioglu A.S. and Demirors O. (2005) A process based model for measuring process quality attributes. In 12 th European Conference on Software Process Improvement (EuroSPI), volume 3792 of LNCS, pages 118-129. Springer, 2005.

Gruhn V., Laue R., (2009), A Heuristic Method for Business Process Model Evaluation, Advances in Enterprise Engineering III, Lecture Notes in Business Information Processing, 2009, Volume 34, Part 1, 28-39, DOI: 10.1007/978-3-642-01915-9_3

Harmon P., (2007), Business process change: a guide for business managers and BPM and six sigma, Elsevier, San Diego, CA, 2007

Harmon P., Wolf C. (2010), The State of Business Process Management - 2010, BPTrends, February 2010, http://www.bptrends.com/members_surveys/deliver.cfm?report_id $=1004 \&$ target=2009 BPTrends State of Market Rept -FINAL PDF CAP 2-1-10.pdf

Held M., Blochinger W. (2008), Structured Collaborative Workflow Design, Future Generation Computer Systems, Volume 25, Issue 6, June 2009, Pages 638-653, ISSN 0167-739X, DOI: 10.1016/j.future.2008.12.005. 
A. 1 Concurrency:

A.1.1 Using a lock when making changes to something shared

Hsueh, N., Shen, W., Yang, Z., \& Yang, D. (2008), Applying UML and software simulation for process definition, verification, and validation. Information and Software Technology. Vol.50, 9-10 (Aug. 2008), pp 897-911.

List, B. \& Korherr, B. (2005). A UML 2 Profile for Business Process Modelling. Perspectives in Conceptual Modeling, Lecture Notes in Computer Science, 2005, Volume 3770/2005, pp 85-96, DOI: 10.1007/11568346_10

Kazanis, P. \& Ginige, A. (2002), Asynchronous collaborative business process modelling through a web forum, 7 th Annual CollECTeR Conference on Electronic Commerce - CollECTeR 02, Melbourne, Australia.

Kettinger, W., Teng, J., Guha, S. (1997), Business Process Change: A Study of Methodologies, Techniques, and Tools, MIS Quarterly, (21: 1); Mar 1997; pg. 55

Koehler J., Vanhatalo J. (2007), Process anti-patterns: How to avoid the common traps of business process modeling IBM WebSphere Developer Technical Journal 10.4., April 2007

Koenig A. (1995) Patterns and Antipatterns. Journal of Object-Oriented Programming, $8(1): 46\{48,1995$.

Krogstie, J., Sindre, G., Jorgensen, H. (2006), Process models representing knowledge for action: a revised quality framework, European Journal of Information Systems, 2006, 15, 91-102. doi:10.1057/palgrave.ejis.3000598

Larsen, T. J., \& Niederman, F. (2005), Causal Mapping for the Investigation of the Adoption of UML in Information Technology Project Development, in V. K. Narayanan and D. J. Armstrong (eds.), Causal Mapping for Research in Information Technology, Harrisburg, PA: Idea Group Publishing, 2005, pp. 233-262.

Larsen, T. J., \& Niederman, F., Limayem, M., Chan, J. (2007), The role of modelling in achieving information systems success: UML to the rescue? Information Systems Journal, Volume 19 Issue 1, Pages 83 - 117, Journal compilation. Blackwell Publishing Ltd.

Laue R., Awad A. (2009) Visualization of Business Process Modeling Anti Patterns First International Workshop on Visual Formalisms for Patterns (VFfP 2009), Electronic Communications of the EASST, 2009 
A.1 Concurrency:

A.1.1 Using a lock when making changes to something shared

Lea, D. (2000), Patterns-Discussion FAQ, Portland Pattern Repository's WikiWikiWeb [Last edited Nov. 2000] http://g.oswego.edu/dl/pd-FAQ/pd-FAQ.html, Retrieved Feb 3, 2011

Lei, B., Wang, L., Li, X. (2008), UML Activity Diagram Based Testing of Java Concurrent Programs for Data Race and Inconsistency, 2008 International Conference on Software Testing, Verification, and Validation, April 2008, pp 200-209

Lin F-R, Yang M-C, Pai Y-H, (2002), A generic structure for business process modeling, Business Process Management Journal, (2002) Vol. 8 Iss: 1, pp.19 - 41

List, B. \& Korherr, B. (2005). A UML 2 Profile for Business Process Modelling. Perspectives in Conceptual Modeling, Lecture Notes in Computer Science, 2005, Volume 3770/2005, 85-96, DOI: 10.1007/11568346_10

Liu R. and Kumar. (2005) A. An Analysis and Taxonomy of Unstructured Workflows, Proceedings of the International Conference on Business Process Management (BPM2005), volume 3649 of Lecture Notes in Computer Science, pages $268\{284$, Nancy, France, 2005. Springer-Verlag.

Mäntylä, M, (2003), Bad Smells in Software - a Taxonomy and Empirical Study, Master's Thesis, Helsinki University Of Technology, 2003

Machado, R. J., Lassen, K. B., Oliveira, S., Couto, M., \& Pinto, P. (2005), Execution of UML Models with CPN Tools for Workflow Requirements Validation. Proc. of the Sixth Workshop and Tutorial on Practical Use of Coloured Petri Nets and the CPN Tools. pp.: 231-250.

Meda H.S., Sen A.K., Bagchi, A. (2010), On Detecting Data Flow Errors in Workflows, ACM Journal of Data and Information Quality, 2, 1, Article 4 (July 2010), 31 pages. DOI $=10.1145 / 1805286.1805290$

Mendling J., Neumann G., van der Aalst W. (2007), On the Correlation between Process Model Metrics and Errors, Tutorials, posters, panels and industrial contributions at the 26th international conference on Conceptual modeling - Volume 83 (ER '07), Vol. 83. Australian Computer Society, Inc., Darlinghurst, Australia, 173-178.

Mendling J., Verbeek H.M.W., van Dongen B.F., van der Aalst W.M.P., Neumann G. (2008) Detection and prediction of errors in EPCs of the SAP reference model, Data \& Knowledge Engineering 64 (2008) 312-329 
A.1 Concurrency:

A.1.1 Using a lock when making changes to something shared

Mendling J., Reijers H.A., van der Aalst W.M.P. (2010), Seven process modeling guidelines (7PMG), Information and Software Technology, Volume 52, Issue 2, February 2010, Pages 127-136, ISSN 0950-5849, DOI: 10.1016/j.infsof.2009.08.004.

Nasiri, R. \& Moenini, A. \& Abdollahzadeh, A. (2004). A New Approach Towards Procurement of Software Models Via Distributed Business Models. The Journal of Supercomputing: 29, 287-302

Onoda, S. Ikkai, Y. Kobayashi, T. Komoda, N. (1999), Definition of Deadlock Patterns for Business Processes Workflow Models, HICSS-32. Proceedings of the 32nd Annual Hawaii International Conference on System Sciences, vol.Track5, no., pp.11 pp.-, 1999

PPR contributors (2010), Code Smell, Portland Pattern Repository, http://www.c2.com/cgi/wiki?CodeSmell (last edited 2, 2010)

Shulman E. L. (2009) basics.html, TiddlyWiki v2.5.0 with BasicsPackage, a TiddlyTools QuickStart $^{\mathrm{TM}}$ document. Last edited Aug.5 2009, Downloaded April 8 2011, http://www.tiddlytools.com/quickstart/basics.html

Przybyłek, A. (2007). The Integration of Functional Decomposition with UML Notation in Business Process Modelling, in Advances in Information Systems Development Ed. Gabor Magyar, Gabor Knapp, Wita Wojtkowski, W. Gregory Wojtkowski and Jože Zupančič, Springer US, 2007, pp 85-99,

Rech, J., Spriestersbach, A. Editors. (2007), Quality Defects in Model-driven Software Development VIDE Consortium (FP6-IST-2004-033606, VIsualize all moDel drivEn programming). Deliverable D4.1, Version 1.0, August 2007, http://www.videist.eu/extern/VIDE_D4.1.pdf

Rising, L., (2000), The Pattern Almanac 2000, Addison-Wesley, 2000

Russell N., ter Hofstede A.H.M., Edmond D. and van der Aalst W.M.P. (2004a), Workflow Data Patterns, QUT Technical report, FIT-TR-2004-01, Queensland University of Technology, Brisbane, 2004.

Russell N., ter Hofstede A.H.M., Edmond D. and van der Aalst W.M.P., (2004b), Workflow Resource Patterns, BETA Working Paper Series, WP 127, Eindhoven University of Technology, Eindhoven, 2004. 
A.1 Concurrency:

A.1.1 Using a lock when making changes to something shared

Russell N., ter Hofstede A.H.M., van der Aalst W.M.P., and Mulyar N., (2006), Workflow Control-Flow Patterns: A Revised View, BPM Center Report BPM-06-22, BPMcenter.org, 2006.

van der Aalst W.M.P., ter Hofstede A.H.M., Kiepuszewski B., and Barros A.P. Workflow patterns. Distributed and Parallel Databases, 14(1):5-51, 2003.

van der Aalst W.M.P., van Hee K.M., ter Hofstede A.H.M. , Sidorova N., Verbeek H.M.W., Voorhoeve M., Wynn M.T. (2008), Soundness of Workflow Nets: Classification, Decidability, and Analysis BPM Center Report BPM-08-02, BPMcenter.org, 2008.

van der Aalst, W.M.P. and ter Hofstede, A.H.M. (2010), Workflow Patterns Home Page http://www.workflowpatterns.com

Sundari M.H., Sen A.K., Bagchi A. (2007), Detecting Data Flow Errors in Workflows: A Systematic Graph Traversal Approach, 17 th Workshop on Information Technology \& Systems (WITS-2007), Montreal, 2007.

Trčka, N., van der Aalst, W., Sidorova, N., (2009), Data-Flow Anti-patterns: Discovering

Data-Flow Errors in Workflows, Advanced Information Systems Engineering, Lecture Notes in Computer Science, 2009 Springer Berlin / Heidelberg

Weber B., Reichert M. (2008), Refactoring Process Models in Large Process

Repositories, Advanced Information Systems Engineering, Lecture Notes in Computer Science, 2008, Volume 5074/2008, 124-139, DOI: 10.1007/978-3-540-69534-9_9

Weiss, M., Amyot, D. (2005), Business Process Modeling with URN, International Journal of E-Business Research. Vol. 1, no. 3, pp. 63-90. July-Sept. 2005

Weiss, M. (2007a), What is a Patlet, Pattern Forge, [Revision as of 16:13, 30 July 2007], http://www.patternforge.net/wiki/index.php?title=What_is_a_Patlet

Weiss M., Birukou, A., (2007b) Building a Pattern Repository: Benefitting from the Open, Lightweight, and Participative Nature of Wikis, Wikis for Software Engineering Workshop (Wikis4SE 2007) October 21, 2007, Montréal, Québec, Canada. 
A.1 Concurrency:

A.1.1 Using a lock when making changes to something shared

\section{A. DATA CONCURRENCY GUIDELINE LIST}

\section{A.1 Concurrency:}

\section{A.1.1 Using a lock when making changes to} something shared

Data Concurrency CBP-2.2

A standard way to avoid concurrency problems is to prevent more than one process or person from making changes to shared data, or access a shared resource, is to lock access to it - like a shared bathroom, until it is available for others to use. This can result in other issues.

\section{A.1.2 Making changes to copied data Data Concurrency WDP-29}

Whenever data is shared by making copies of it, then it is very easy for the copies to cease being the same.

\section{A.1.3 Concurrency management Data Concurrency WDP-29}

Different modeling languages have many ways to manage local and remote concurrent access to data and resources, including doing nothing.

\section{A.1.4 Accessing shared data Data Concurrency WDP-5}

\section{A.2 External:}

\section{A.2.1 Sending data outside of the workflow Data External WDP-15}

Sending data into the environment (outside the Workflow/BPM)

\section{A.2.2 Receiving data or requests from outside} workflow

Data External WDP-17

The outside environment can push data into the workflow at any time. Similarly, when the outside environment may ask at any time for information from the 
A.3 Intemal:

A.2.3 Retrieving data from outside of the workflow

Name

Categ. Section

Source

workflow

\section{A.2.3 Retrieving data from outside of the} workflow

Data External WDP-16

The workflow needs to retreive information from the outside world.

\section{A.3 Internal:}

\section{A.3.1 Data used by multiple instances $\quad$ Data Internal WDP-1}

Multiple instances of a process use the same data, for example multiple releases of a product all using the same installation manual.

\section{A.3.2 Separate data and control flow channels ${ }_{D a t a} \quad$ Internal $\quad$ WDP-9}

Control and data travel separately to tasks. For example tasks are triggered by a schedule but the data that needs to be processed is sent electronically (or through the mail, etc.)

\section{A.3.3 Merging results from multiple instance tasks Data Internal WDP-12}

When multiple instances of a sub-process have finished does their data need to be merged back together in order to continue?
A.3.4 Shared case data
Data Internal
WDP-14

Sharing data between two related instances of a workflow (i.e. separate but related cases)

\section{A.3.5 Bypassing gateways \\ Data Internal PAP-5.6}

Data bypasses gateways because it is not needed until some later point in the execution 
A.4 Robustness:

A.3.6 Dangling inputs and outputs

Name

Categ. Section

Source

\section{A.3.6 Dangling inputs and outputs Data Internal PAP-5.1}

Inputs and outputs of an activity or gateway that remain unconnected in the model, very often as residues of connections that users decide to delete or redirect. (Many BPM tools/languages will catch this automatically, but not all)

\section{A.4 Robustness:}
A.4.1 Existence check
Data Robustness
WDP-34

Checking if a value or object currently exists.

\section{A.5 Routing:}

\section{A.5.1 Data-based routing Data Routing WDP-40}

When the evaluation of data-based expressions have the ability to alter the controlflow within a case.

\section{A.6 State:}

\section{A.6.1 Using the token design pattern $\quad$ Data $\quad$ State $\quad$ CBP-4.2}

A token is an exclusively held resource that is analogous to a physical object. Only one party can hold a token at any given time and if the token is destroyed, it cannot be used at all. This pattern is crucial to how many modeling languages work (e.g. BPMN), however the pattern can be used within a Business Processes as well, for example: in formal meetings only the person who "has the floor" is supposed to speak. 
A.7 Termination:

A.7.1 Multiple termination points

Name

Categ. Section

Source

\section{A.7 Termination:}

\section{A.7.1 Multiple termination points Control Termination PAP-7.1}

(also known as: Stop Node in Parallel Execution Branches)

In some modeling languages there are stop nodes which shutdown the whole process as well as end nodes which just terminate an execution branch. The stop nodes may also be needed to release data from a sub-process to its parent process when it finishes. Therefore a model might contain stop/shutdown nodes on multiple branches.

\section{A.7.2 Not cleaning up data \\ Data Termination \\ DAP-7}

Data is not cleaned up after use, or ever

\section{A.8 Transfer:}

\section{A.8.1 Passing data to a different data type Data Transfer WDP-32}

The Sender and Receiver use different data types when passing information

\section{A.9 Trigger:}

\section{A.9.1 Events as control flow \\ Data Trigger \\ PAP-6.1}

Frequently control flow is used to capture events.

\section{A.10 Usability:}

\section{A.10.1 Multiple connections between activities Data Usability PAP-5.3}

Complex control and data flows easily lead to multiple connections in process models, which can result in cluttered models. 
A.11 Usage:

A.10.2 Cluttercd data-flow models

Name

Categ. Section

Source

$\begin{array}{lll}\text { A.10.2 Cluttered data-flow models } & \text { Data } & \text { Usability }\end{array}$

\section{A.11 Usage:}

A.11.1 Data is written to, but never used Data Usage DAP-2

A.11.2 Data is over-written without being used first

Data Usage DAP-4

A.11.3 Data is read or destroyed before being initialized or written to.

Data Usage

DAP-1

\section{A.12 Visibility:}

\section{A.12.1 Reusing names for data \\ Data Visibility \\ WDP-1}

Reusing names for data elements in different parts of the process model. 
B. I Concurrency:

B.1.1 Using a lock when making changes to something shared

\section{B. DATA CONCURRENCY GUIDELINES AND ISSUES (DEPTH OF 3)}

\section{B.1 Concurrency:}

\section{B.1.1 Using a lock when making changes to something shared Data Concurrency CBP-2.2}

A standard way to avoid concurrency problems is to prevent more than one process or person from making changes to shared data, or access a shared resource, is to lock access to it - like a shared bathroom, until it is available for others to use. This can result in other issues.

Consider Issue:

Object assumed to be protected

Control Concurrency

CBP-2.2

(also known as: Code assumed to be protected)

Assuming access by others is prevented when it actually isn't (like people who assume a bathroom door is always locked when the bathroom is in use)

$\begin{array}{llll}\text { Wrong lock or no lock } & \text { Control Concurrency } & \text { CBP } \\ & & \end{array}$

If access to something is only controlled by an agreed upon "protocol" that has no way to absolutely control that access, then problems may occur when the protocol is not followed correctly or at all. For example people who are used to locked bathroom doors showing occupancy vs people who expect others to knock before opening the door.

Non-protected version is used instead of protected version

If not all the ways to access something are protected then using the unprotected access (unnecessarily) may cause problems. For example always accessing a computer through an administrator (or root) account can cause a lot of problems. 
B.1 Concurrency:

B.1.1 Using a lock when making changes to something shared

Related to Pattern:

Protection and subclassing

(also known as: Confinement and Subclassing)

\section{Double-checked locking}

Data

Creation

CBP-

2.2 .4

This comes from software, where a newly created object is available for others to use before it has been completely initialized. Similarly a report could be released for others to use while the author is still gathering information.

\section{Nonatomic operations assumed to be atomic Control Concurrency 2.2 .1}

Sometimes an activity that looks like it cannot be interrupted (self contained, i.e. atomic) can be because of the underlying activities which implement it. For example asking one person to sign a report not realizing they are really the contact person into an organization with its own approval process.

\section{Consider Pattern:}

Critical section

$\begin{array}{lll}\text { Control } & \text { State } & \text { WCP-39 } \\ \text { Control } & \text { State } & \text { WCP-40 } \\ \text { Data } & \text { Transfer } & \text { WDP-31 } \\ & & \text { CBP- } \\ \text { Control } & \text { Concurrency } & 2.2 .2\end{array}$

Two-stage access bug pattern Concurrency

$\mathrm{CBP}$

Sometimes a sequence of operations needs to be protected but instead it was wrongly assumed that protecting each operation separately is enough. For example transferring money electronically from one bank to another. It is not desirable if at any time that person appeared to have twice the amount of money or none.

\section{Transfer is not atomic.}

Data Transfer

CBP-4.2

If control of something depends having possession of an object (for example a baton, or a crown) but the object is only exists digitally (e.g. a token), then it is possible for that object to exist in more than one place or cease to exist 
B. 1 Concurrency:

B.1.1 Using a lock when making changes to something shared

completely. For example, when transferring money electronically, the value must be deleted from one account and added to another. During this time, or if something went wrong, then the money could be in two accounts or no longer existing at all.

\section{Consider Solution:}

Clean-up after transfer

$$
\text { Data Transfer }
$$

\section{Related to Pattern:}

The token design pattern

Data State

CBP-4.2

\section{Related to Smell:}

Using the token design pattern Data State $\quad$ CBP-4.2

\section{Contention problems with shared resources Data Transfer WDP-29}

Components of a process may find it difficult to function independently of data changes and concurrency constraints that occur in the broader process environment. For example: other components make changes to shared data that impacts this component (which needs the data to be stable), or other components lock access to shared data preventing this component from making the changes it needs.

\section{Consider Pattern:}

Transfer - copy in/copy out

Data Transfer

WDP-29

Transfer by value - outgoing

Data Transfer

WDP-28

Transfer by value - incoming

Data Transfer

WDP-27

\section{Consider Solution:}

Version control

Data Visibility

tbd

\section{Related to Smell:}

Concurrency management

Data Concurrency

WDP-29 
B.l Concurrency:

B.1.2 Making changes to copied data

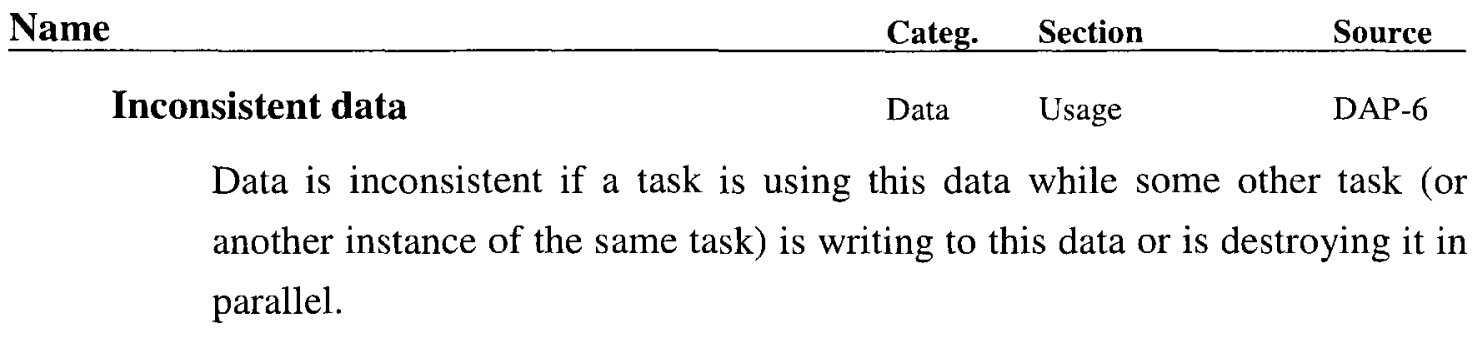

\section{B.1.2 Making changes to copied data Data Concurrency WDP-29}

Whenever data is shared by making copies of it, then it is very easy for the copies to cease being the same.

\section{Consider Issue:}

Passing data when control flow and data travel together Data Internal WDP-9

(also known as: Passing data when control and data channels are integrated)

If the data for an activity and control of the activity are integrated, and if the data was copied to parallel paths, then it is possible that two (or more) different copies of the data will arrive at the same location. For example while building a house, copies of the plans are passed along to each new trade as they take over an area - along with notes, changes, deletions and possibly mistakes. Eventually someone will receive two copies of the plans which are no longer the same.

\section{Consider Solution:}

Merge changes strategy

Data Transfer

WDP-29

\section{Related to Pattern:}

Task to task

Data Internal

WDP-9

Unknowable outcome when copied data is updated

Data Transfer

WDP-29

Difficulties can arise where data is passed to a sub-activity that executes independently of the original activity. If these activities continue in parallel, then there is no way to know for certain when the sub-activity will end and 
B. 1 Concurrency:

B.1.3 Concurrency management

will copy its data back to the original. If the data has changed then it is important to know what impact this will have on the original (since you don't know when it will happen).

\section{Consider Solution:}

No asynchronous sub-routine calls

$\begin{array}{lll}\text { Data } & \text { Transfer } & \text { WDP-29 } \\ \text { Data } & \text { Transfer } & \text { WDP-29 } \\ \text { Data } & \text { Transfer } & \text { WDP-29 }\end{array}$

Do not copy data back

Merge changes strategy

$$
\text { Data Transfer }
$$

WDP-28

Transfer by value - outgoing

$\begin{array}{lll}\text { Data } & \text { Transfer } & \text { WDP-28 } \\ \text { Data } & \text { Transfer } & \text { WDP-29 } \\ \text { Data } & \text { Transfer } & \text { WDP-27 }\end{array}$

Transfer - copy in/copy out

Data Transfer

WDP-29

\section{B.1.3 Concurrency management}

Data Concurrency

Different modeling languages have many ways to manage local and remote concurrent access to data and resources, including doing nothing.

\section{Consider Issue:}

\section{Common datastore concurrent access Data Concurrency WDP-5}

Where a common data store is utilized by several tasks for communicating data elements (rather than communicating the data between them directly), there is the potential for concurrency problems to arise, particularly if the case involves parallel execution paths. This may lead to inconsistent results depending on the task execution sequence that is taken.

\section{Consider Solution:}

No asynchronous sub-routine calls

$\begin{array}{lll}\text { Data } & \text { Transfer } & \text { WDP-29 } \\ \text { Data } & \text { Concurrency } & \text { WDP-9 }\end{array}$

Language specific serialization

$$
\text { Data }
$$

Data Visibility

WDP-2 
B. 1 Concurrency:

B.1.3 Concurrency management

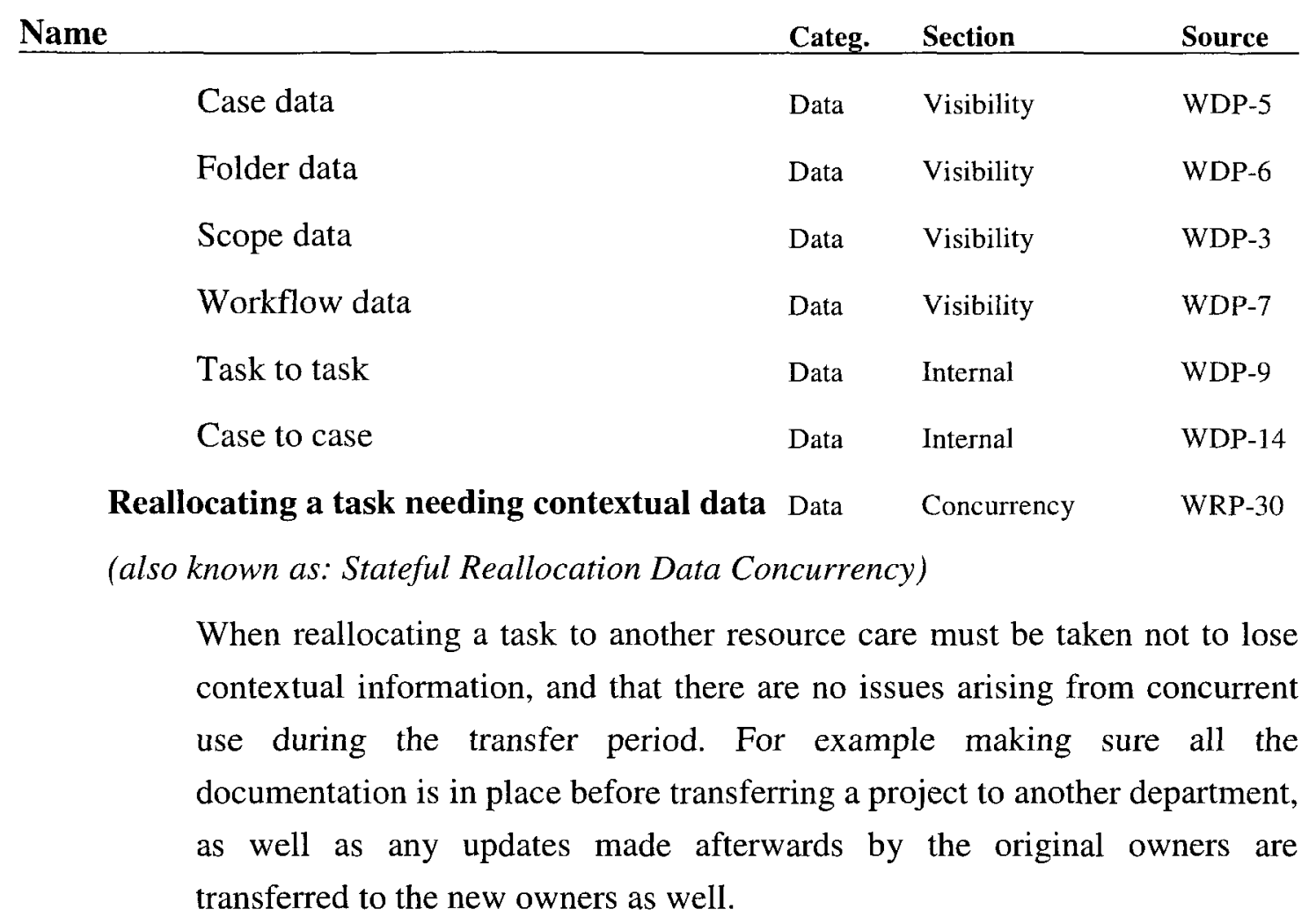

\section{Consider Pattern:}

Stateless reallocation

Resource Detour

WRP-31

Delegation

Resource Detour

WRP-27

Stateful reallocation

Resource Detour

WRP-30

\section{Consider Solution:}

Stateful reallocation data concurrency Resource Concurrency WRP-30

Random problems Control Concurrency WCP-1

Mismanaging concurrency can cause different results depending on what order activities happen. Issues may appear as seemingly random behaviour. Data may be lost or corrupted, but only on rare occasions. They can also cause performance problems, or create holes that larger problems may escape through unnoticed until much later. 
B.1 Concurrency:

B.1.3 Concurrency management

Name

Categ. Section

Source

Consider Solution:

Multiple tokens

Control Concurrency

WCP-1

Safe process model

Control Concurrency

WCP-1

Related to Pattern:

Sequence

Control Basic

WCP-1

(also known as: Sequential routing, serial routing.)

Something tries to access results before ready

Data Internal

CBP -4.3

(also known as: Task tries to access results before ready)

For example someone not checking if a report is finished before making it public.

\section{Consider Solution:}

Use join to wait.

Control Synchronizor

CBP-

2.3.1

\section{Related to Pattern:}

The fork/join design pattern

Control Synchronizor

CBP-4.3

The sleep bug pattern

Control Synchronizor

2.3.1

Essentially assuming that one activity is faster than another so adding a delay to synchronize them, when in fact there is no guarantee one will be faster than the other (e.g. The fable of the Hare taking a nap because he thought he had plenty of time to win the race versus the Tortoise). Mostly it is a performance problem, but it can also cause race conditions with seemingly random results.

\section{Consider Solution:}

Use join to wait.

Control Synchronizor

CBP-

Use join to wait.

Assume concurrency will never occur

Control Concurrency

2.3.1

(also known as: Interleavings assumed never to occur) 
B.1 Concurrency:

B.1.4 Accessing shared data

The assumption that certain activities will never happen in parallel so there is no need to prevent them (e.g. two people updating an on-line document at the same time).

\section{Losing a notify bug pattern}

When depending on messages to control access to a shared object, and not everyone is listening when the message is sent

Contention problems with shared resources Data Transfer WDP-29

PLEASE SEE earlier description

\section{Subprocesses can access everything Data Visibility WDP-10}

Information is accessible by everything as soon as it is available, so data is passed to other tasks simply by being made available, hence concurrent tasks may try to make changes to the same data at unexpected times.

\section{Consider Pattern:}

Block data

Data Visibility

WDP-2

\section{Related to Smell:}

Reusing names for data

Data Visibility

WDP-1

\section{Related to Solution:}

Implicit data passing

Data Internal

WDP-10

\section{B.1.4 Accessing shared data}

Data Concurrency

WDP-5

\section{Consider Issue:}

Case data access

Data Visibility

WDP-5

One consideration that arises with the use of case level data is in ensuring that these elements are accessible, where required, to the components of a subprocess associated with a specific case (e.g. as the definition of a block task). 
B.I Concurrency:

B.1.4 Accessing shared data

Name

Categ. Section

Source

Consider Solution:

Explicit case data access

Data Visibility

WDP-5

Implicit case data access

Data Visibility

WDP-5

Related to Pattern:

Case data

Data Visibility

WDP-5

Multiple instance data availability

Data Internal

WDP-13

One issue that arises with multiple instance tasks is identifying the point at which the output data from them are available (in some aggregate form) to subsequent tasks.

\section{Related to Pattern:}

From multiple instance task

$\begin{array}{lll}\text { Data } & \text { Internal } & \text { WDP-13 } \\ \text { Data } & \text { Visibility } & \text { CBP-4.3 }\end{array}$

\section{Result not available after join}

When an activity has several tasks happening in parallel, changes may be made to data which is lost when the separate tasks join back together. For example, having a document updated by several people in parallel but not ensuring that all those changes have been made it into the final version.

\section{Consider Pattern:}

Transfer by reference - with lock

Data Transfer

WDP-31

Critical section

Control State

WCP-39

Interleaved routing

Control State

WCP-40

Consider Solution:

Use higher scope of variables for task

Data Visibility

WDP

\section{Related to Pattern:}

The fork/join design pattern 
B.2 External:

B.2.1 Sending data outside of the workflow

Name

Categ. Section

Source

\section{B.2 External:}

\section{B.2.1 Sending data outside of the workflow}

Data External

WDP-15

Sending data into the environment (outside the Workflow/BPM)

\section{Consider Issue:}

Assuring data delivery

Data External

WDP-15

When sending data to an external application how do you know if it was successfully delivered? This is particularly a problem when the external application does not provide immediate feedback on delivery status.

\section{Consider Solution:}

Delivery verification

Data External

WDP-15

Delivery acknowledgement

Data External

WDP-15

\section{Related to Pattern:}

Task to environment - push

Data External WDP-15

Case to environment - push

Data External

WDP-19

Environment to case - pull

Data External

WDP-20

Environment to task - pull

Data

External

WDP-16

Environment to workflow - pull

Data Externa

WDP-24

Workflow to environment - push

Data

External

WDP-23

\section{Appropriate time to push}

Data External

WDP-19

Determining the proper time to push the data outside to the environment.

\section{Consider Solution:}

Rule-based communication scheduling Data Termination $\quad$ WDP-19 
B.2 External:

B.2.2 Receiving data or requests from outside workflow

Related to Pattern:

Task to environment - push

Data External

WDP-15

Case to environment - push

Data External

WDP-19

Environment to case - pull

Data External

WDP-20

Environment to task - pull

Data External

WDP-16

Environment to workflow - pull

Data External

WDP-24

Workflow to environment - push

Data

External

WDP-23

\section{B.2.2 Receiving data or requests from outside workflow}

Data External

WDP-17

The outside environment can push data into the workflow at any time. Similarily, when the outside environment may ask at any time for information from the workflow

\section{Consider Issue:}

\section{Data source identification}

Data

External

WDP-17

Is there any way for an outside application to know who within the over-all workflow it needs to send information to (or ask for information from)

\section{Consider Solution:}

Inform environment

Data External

WDP-17

\section{Related to Pattern:}

Environment to task - push

WDP-17

Task to environment - pull

Data External

WDP-18

Case to environment - pull

Data External

WDP-22

Workflow to environment - pull

Data

External

WDP-26

Environment to case - push

Data External

WDP-21

Environment to workflow - push

Data External

WDP-25 
B.2 External:

B.2.2 Receiving data or requests from outside workflow

Name
Need additional data path for returned info. Data
If data can be sent to the workflow at any time, then the actual
implementation of the process model needs some mechanism for the data to
arrive via. Especially if it was something in the workflow that requested the
information, but is not passively waiting for it (i.e. it continues to execute
other things rather than stopping and waiting)

\section{Consider Solution:}

Asynchronous communication facilities Data External WDP-17

\section{Related to Solution:}

Don't wait for data response Data External WDP-16

Wait and respond to data requests without

impacting progress Data External WDP-17

If data can be sent to the workflow at any time then the workflow needs a way to know it has arrived without impacting the workflow's performance (e.g. by stopping or continually checking for it.)

\section{Consider Solution:}

Asynchronous communication facilities Data External WDP-17

\section{Related to Pattern:}

Environment to task - push Data External WDP-17

Task to environment - pull Data External WDP-18

Case to environment - pull Data External WDP-22

Workflow to environment - pull Data External WDP-26

Environment to case - push Data External WDP-21

Environment to workflow - push Data External WDP-25

Data notification Data External WDP-21

During the course of the execution of a case, new data may become available 
B.2 External:

B.2.3 Retrieving data from outside of the workflow

or the values of existing items may change. A means of communicating these data updates to the appropriate case is required.

\section{Consider Solution:}

Data notification at start of case

Data External WDP-21

Provide mechanism to update case data

\section{Data}

External

WDP-21

\section{Related to Pattern:}

Environment to case - push

Data External

WDP-21

Environment to workflow - push

Data External

WDP-25

Case to environment - pull

Data

External

WDP-22

Workflow to environment - pull

Data External

WDP-26

\section{B.2.3 Retrieving data from outside of the workflow}

Data External

WDP-16

The workflow needs to retrieve information from the outside world.

\section{Consider Issue:}

$\begin{array}{llll}\text { Request prevents progress Data External WDP-16 } & \text { Wal }\end{array}$

This style of interaction can block progress of the requesting case if the external application has a long delivery time for the required information or is temporarily unavailable.

\section{Consider Solution:}

Don't wait for data response

Data External

WDP-16

\section{Related to Pattern:}

Environment to task - pull

Data External

WDP-16

Environment to case - pull

Data External

WDP-20

Case to environment - push

Data External

WDP-19

Task to environment - push

Data External

WDP-15 
B.2 External:

B.2.3 Retrieving data from outside of the workflow

\begin{tabular}{rlll} 
Name & Categ. & Section & Source \\
\hline Workflow to environment - push & Data & External & WDP-23 \\
Environment to workflow - pull & Data & External & WDP-24 \\
Appropriate time to pull & Data & External & WDP-20
\end{tabular}

Another issue is determining the best time for both a particular case and the environment to pull in data from outside the workflow..

\section{Consider Solution:}

Beginning of task or case

Data External

WDP-20

\section{Related to Pattern:}

Environment to task - pull

Data External WDP-16

Environment to case - pull

Data External WDP-20

Case to environment - push

Data External

WDP-19

Task to environment - push

Data External

WDP-15

Workflow to environment - push

Data

External

WDP-23

Environment to workflow - pull

Data External

WDP-24

\section{Requires explicit support for the extraction} of data from outside

Data External WDP-20

The implementation of the workflow needs to actually be capable of extracting data from external applications.

\section{Consider Solution:}

Database mapping functions

Data External

WDP-20

\section{Related to Pattern:}

Environment to task - pull

Dat

External

WDP-16

Environment to case - pull

Data External

WDP-20

Case to environment - push

Data

External

WDP-19

Task to environment - push

Data External

WDP-15 
B.3 Internal:

B.3.1 Data used by multiple instances

Name

Categ. Section

Source

Workflow to environment - push

Data

External

WDP-23

Environment to workflow - pull

Data External

WDP-24

\section{B.3 Internal:}

\section{B.3.1 Data used by multiple instances Data Internal WDP-1}

Multiple instances of a process use the same data, for example multiple releases of a product all using the same installation manual.

\section{Consider Issue:}

Multiple instance data initialization Data Internal WDP-1

When a task is able to execute multiple times but not all instances are created at the same time, then are the values of data elements for all execution instances set when the multiple instance task is initiated or can they be set after this occurs for each invocation of a task instance.

\section{Related to Pattern:}

$\begin{array}{llll}\text { Sequence } & \text { Control } & \text { Basic } & \text { WCP-1 }\end{array}$

(also known as: Sequential routing, serial routing.)

Task data

Data Visibility

WDP-1

To multiple instance task

Data Internal

WDP-12

\section{B.3.2 Separate data and control flow channels}

Data Internal WDP-9

Control and data travel separately to tasks. For example tasks are triggered by a schedule but the data that needs to be processed is sent electronically (or through the mail, etc.) 
B.3 Internal:

B.3.2 Separate data and control flow channels

\section{Consider Issue:}

Data is late

Data Internal

WDP-9

When data and control-flow occur via separate communication channels, then is possible the control-flow reaches a task before the required data elements have been passed to it. For example, your utility payment is due, but the cheque is still in the mail.

\section{Consider Solution:}

Wait for data

Control Trigger

WDP-9

Wait to be processed

Control Trigger

WCP3

Related to Pattern:

Task to task

Data Internal

WDP-9

\section{Related to Solution:}

Explicit data passing via data channels Data Internal WDP-10

Bypassing synchronizing gateways Data Internal PAP-5.6

Dataflow bypassing synchronization gateways of alternative branches can cause deadlocks because data needed by a future operation may not be created along some branch Note: Bypassing forks and joins in parallel flows does not cause a deadlock, because all branches execute in parallel and thus, all business items always arrive through the connections i.e., all tasks receive their inputs as specified.

\section{Consider Solution:}

Make input optional

Data Transfer

PAP-5.7

\section{Related to Smell:}

Bypassing gateways

Data Internal

PAP-5.6

Related to Solution:

Explicit data passing via data channels

Data Internal

WDP-10 
B.3 Internal:

B.3.2 Separate data and control flow channels

Name

Categ. Section

Source

A thread of control never arrives (lost tokens)

Control Robustness WCP-3

The use of the Synchronization pattern can potentially give rise to a deadlock in the situation where one of the incoming branches fails to deliver a thread of control to the join construct.

\section{Consider Solution:}

Structured process (no

interrupts/exceptions)

Control Usage

WCP-3

Cancel region

Control Cancellation

WCP-29

\section{Related to Pattern:}

Synchronization (m-of-m and-join) Control Synchronizor $\quad$ WCP-3

(also known as: AND-join, rendezvous, synchronizer)

Thread merge (and-join) Control Discriminator $\quad$ WCP-41

Structured synchronizing merge (or-join) Control Synchronizor $\quad$ WCP-7

(also known as: Synchronizing join, synchronizer, OR-join)

Generalized and-join (m-of-m and-join) Control Synchronizor WCP-33

Local synchronizing merge (or-join) Control Synchronizor WCP-37

\section{Detecting undefined vs empty/null Data Robustness WDP-34}

Being able to differentiate between data elements that have an undefined value and those that are merely empty or null.

\section{Consider Solution:}

Deciding undefined vs empty/null

Data Visibility

WDP-34

\section{Related to Pattern:}

Task precondition - data existence

Data Routing

WDP-34

Task postcondition - data existence

Data

Routing

WDP-36

Task postcondition - data value

Data Routing

WDP-37 
B.3 Internal:

B.3.2 Separate data and control flow channels

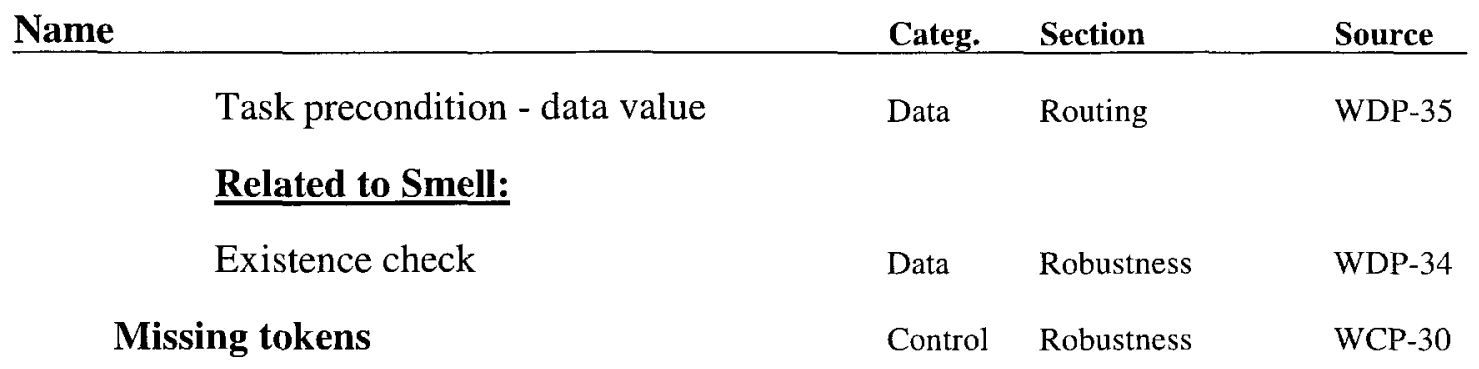

Individual abended threads/tokens would prevent a Join from reseting and failure to receive input on all of the incoming branches may result in the process instance (and possibly other process instances) stalling. Missing tokens could be a consequence of: - a design error - or that one of the tasks in the branch failing to complete successfully (e.g. as a consequence of it experiencing some form of exception) - or because the thread of control is passed outside of the branch. - or there are preceeding splits or joins or they are un-structured in form (i.e. un-balanced splits and joins) (from WCP3)

Consider Solution:

Cancel region

Control Cancellation

WCP-29

Cancelling partial join (n-of-m and-join) Control Robustness

WCP-30

\section{Related to Pattern:}

Structured partial join (n-of-m and-join) Control

Discriminator

WCP-30

Structured discriminator (1-of-m andjoin)

Control Discriminator

WCP-9

(also known as: 1-out-of-M join.)

Static partial join for multiple instances (n:m \&-join)

Control Multiple_instance

WCP-34

Multiple instances with a priori run-time knowledge (m:m and-join)

Control Multiple_instance

WCP-14

Multiple instances with a priori designtime knowledge ( $\mathrm{m}: \mathrm{m}$ and-join)

Control Multiple_instance

WCP-13

Multiple instances without a priori runtime knowledge

Control Multiple_instance WCP-15 
B.3 Internal:

B.3.2 Separate data and control flow channels

Name

Categ. Section

Source

Dynamic partial join for multiple

instances

Control Multiple_instance

WCP-36

Blocking partial join (n-of-m and-join)

Control

Discriminator

WCP-31

Blocking discriminator (1-of-m and-join) Control

Discriminator

WCP-28

Cancelling partial join for multiple

instances (n:m and-join)

Control Multiple_instance

WCP-35

Related to Smell:

Using the token design pattern

Data State

CBP-4.2

Discriminator missing tokens

Control Robustness

WCP-9

Individual abended threads/tokens would prevent a Discriminator from resetting and failure to receive input on all of the incoming branches may result in the process instance (and possibly other process instances) stalling.

Consider Pattern:

Cancelling discriminator (1-of-m andjoin)

Control Discriminator

WCP-29

Cancelling partial join (n-of-m and-join) Control

Discriminator

WCP-32

\section{Related to Pattern:}

Structured discriminator (1-of-m andjoin)

(also known as: 1-out-of-M join.)

Structured partial join (n-of-m and-join)

Control

Discriminator

WCP-30

Static partial join for multiple instances

(n:m \&-join)

Control Multiple_instance

WCP-34

Multiple instances with a priori run-time knowledge ( $\mathrm{m}: \mathrm{m}$ and-join)

Control Multiple_instance

WCP-14

Multiple instances with a priori designtime knowledge (m:m and-join)

Control Multiple_instance WCP-13 
B.3 Internal:

B.3.3 Merging results from multiple instance tasks

Name

Categ. Section

Source

Multiple instances without a priori run-

time knowledge

Control Multiple_instance WCP-15

Dynamic partial join for multiple

instances

Control Multiple_instance WCP-36

Blocking discriminator (1-of-m and-join) Control

Discriminator

WCP-28

Blocking partial join (n-of-m and-join) Control Discriminator WCP-31

Cancelling partial join for multiple

instances (n:m \&-join)

Control Multiple_instance

WCP-35

Passing data to subprocesses

Data Internal

WDP-10

The actual implementation of a process needs a way to make data available to its sub-processes

\section{Consider Solution:}

Implicit data passing

Data Internal

WDP-10

Explicit data passing via parameters

Data Internal

WDP-10

Explicit data passing via data channels

Data Internal

WDP-10

\section{Related to Pattern:}

Block task to sub-workflow

decomposition

Data Internal

WDP-10

Sub-workflow decomposition to block task

Data Internal WDP-11

\section{B.3.3 Merging results from multiple instance tasks}

Data Internal

WDP-12

When multiple instances of a sub-process have finished does their data need to be merged back together in order to continue?

\section{Consider Issue:}

Merging results Data Internal WDP-12

When instance specific data is passed by value (i.e. copies), then if at the 
B.3 Internal:

B.3.4 Shared case data

Name

Categ. Section

Source

conclusion of the tasks the data needs to be reassembled then the data may not go back to together as easily as it came appart. For example after each contributor has updated their copy of the report, all the changes need to be merged back together.

Consider Solution:

Shared data passed by reference

Data Internal

WDP-12

Merge changes strategy

Data Transfer

WDP-29

Related to Pattern:

From multiple instance task

Data Internal

WDP-13

\section{B.3.4 Shared case data}

Data Internal

WDP-14

Sharing data between two related instances of a workflow (i.e. separate but related cases)

\section{Consider Issue:}

\section{Linking shared case data}

Data Internal

WDP-14

Establishing an effective means of linking related cases and their associated data together in a meaningful way.

\section{Consider Solution:}

Shared case data support

$$
\text { Data Internal }
$$

WDP-14

\section{Related to Pattern:}

Case to case

Data Internal

WDP-14

\section{B.3.5 Bypassing gateways}

Data Internal

PAP-5.6

Data bypasses gateways because it is not needed until some later point in the execution 
B.3 Internal:

B.3.6 Dangling inputs and outputs

Name

Categ. Section

Source

Consider Issue:

Bypassing synchronizing gateways

Data Internal

PAP-5.6

PLEASE SEE earlier description

\section{B.3.6 Dangling inputs and outputs Data Internal PAP-5.1}

Inputs and outputs of an activity or gateway that remain unconnected in the model, very often as residues of connections that users decide to delete or redirect. (Many BPM tools/languages will catch this automatically, but not all)

\section{Consider Issue:}

Dangling inputs can cause task to hang Data Internal PAP-5.1

Dangling inputs are often the source of simulation errors or prevent the simulation from running all, because an activity or gateway waits for some input that it can never receive.

\section{Related to Solution:}

Use dangling inputs and outputs to reduce clutter.

Data Usability

PAP-5.2

Dangling output means lost data

Data Internal

PAP-5.1

Dangling data outputs show that a task or subprocess produced some data but this data is not used subsequently in the process.

\section{Related to Solution:}

Use dangling inputs and outputs to reduce clutter.

Data Usability

PAP-5.2

\section{Strongly redundant data}

Data Usage

DAP-2

A data element is strongly redundant if there is a writing activity after which in all possible continuations of the execution is completed. Strongly redundant data indicates in most situations a real flaw in the workflow. 
B.4 Robustness:

B.4.1 Existence check

\section{Related to Smell:}

Data is written to, but never used Data Usage DAP-2

Weakly redundant data Data Usage DAP-3

A data element is weakly redundant if there is some execution scenario in which it is written but never read afterwards, i.e. before it is destroyed or the total case's execution is completed. Weakly redundant data is not necessarily a flaw. For example, to improve performance some data may be computed in advance, even though it may not be needed later.

Related to Smell:

Data is written to, but never used Data Usage DAP-2

\section{B.4 Robustness:}

\section{B.4.1 Existence check Data Robustness}

Checking if a value or object currently exists.

\section{Consider Issue:}

\section{Detecting undefined vs empty/null Data Robustness $\quad$ WDP-34}

Being able to differentiate between data elements that have an undefined value and those that are merely empty or null.

\section{Consider Solution:}

Deciding undefined vs empty/null

Data Visibility

WDP-34

\section{Related to Pattern:}

Task precondition - data existence

Data Routing

WDP-34

Task postcondition - data existence

Data

Routing

WDP-36

Task postcondition - data value

Data Routing

WDP-37

Task precondition - data value

Data Routing

WDP-35 
B.4 Robustness:

B.4.1 Existence check

Name

Categ. Section

Source

A thread of control never arrives (lost tokens)

Control Robustness

WCP-3

The use of the Synchronization pattern can potentially give rise to a deadlock in the situation where one of the incoming branches fails to deliver a thread of control to the join construct.

\section{Consider Solution:}

Structured process (no

interrupts/exceptions)

Control Usage

WCP-3

Cancel region

Control Cancellation

WCP-29

Related to Pattern:

Synchronization (m-of-m and-join) Control Synchronizor $\quad$ WCP-3

(also known as: AND-join, rendezvous, synchronizer)

Thread merge (and-join) Control Discriminator $\quad$ WCP-41

Structured synchronizing merge (or-join) Control Synchronizor $\quad$ WCP-7

(also known as: Synchronizing join, synchronizer, OR-join)

Generalized and-join (m-of-m and-join) Control Synchronizor $\quad$ WCP-33

Local synchronizing merge (or-join) Control Synchronizor $\quad$ WCP-37

Missing tokens Control Robustness WCP-30

Individual abended threads/tokens would prevent a Join from resetting and failure to receive input on all of the incoming branches may result in the process instance (and possibly other process instances) stalling. Missing tokens could be a consequence of: - a design error - or that one of the tasks in the branch failing to complete successfully (e.g. as a consequence of it experiencing some form of exception) - or because the thread of control is passed outside of the branch. - or there are preceding splits or joins or they are un-structured in form (i.e. un-balanced splits and joins) (from WCP3) 
B.4 Robustness:

B.4.1 Existence check

\section{Consider Solution:}

Cancel region $\quad$ Control Cancellation $\quad$ WCP-29

Cancelling partial join (n-of-m and-join) Control Robustness $\quad$ WCP-30

\section{Related to Pattern:}

Structured partial join (n-of-m and-join) Control Discriminator WCP-30

Structured discriminator (1-of- $\mathrm{m}$ and-

join)

Control Discriminator WCP-9

(also known as: 1-out-of-M join.)

Static partial join for multiple instances (n:m \&-join)

Control Multiple_instance WCP-34

Multiple instances with a priori run-time knowledge (m:m \&-join)

Control Multiple_instance

WCP-14

Multiple instances with a priori designtime knowledge ( $\mathrm{m}: \mathrm{m} \&$-join)

Control Multiple_instance

WCP-13

Multiple instances without a priori run-

time knowledge

Control Multiple_instance

WCP-15

Dynamic partial join for multiple

instances

Control Multiple_instance WCP-36

Blocking partial join (n-of-m and-join)

Contro

Discriminator

WCP-31

Blocking discriminator (1-of-m and-join) Control

Discriminator

WCP-28

Cancelling partial join for multiple

instances (n:m \&-join)

Control Multiple_instance

WCP-35

\section{Related to Smell:}

Using the token design pattern

Data State

CBP-4.2

\section{Bypassing synchronizing gateways}

Data Internal

PAP-5.6

Dataflow bypassing synchronization gateways of alternative branches can cause deadlocks because data needed by a future operation may not be created along some branch Note: Bypassing forks and joins in parallel flows 
B.4 Robustness:

B.4.1 Existence check

does not cause a deadlock, because all branches execute in parallel and thus, all business items always arrive through the connections i.e., all tasks receive their inputs as specified.

\section{Consider Solution:}

Make input optional

Data Transfer

PAP-5.7

Related to Smell:

Separate data and control flow channels

$\begin{array}{lll}\text { Data } & \text { Internal } & \text { WDP-9 } \\ \text { Data } & \text { Internal } & \text { PAP-5.6 }\end{array}$

Bypassing gateways

Data Internal

WDP-10

Explicit data passing via data channels

Data Internal

WCP-9

Discriminator missing tokens

Control Robustness

WCP-9

Individual abended threads/tokens would prevent a Discriminator from resetting and failure to receive input on all of the incoming branches may result in the process instance (and possibly other process instances) stalling.

\section{Consider Pattern:}

Cancelling discriminator (1-of-m andjoin)

Control Discriminator

WCP-29

Cancelling partial join (n-of-m and-join) Control

Discriminator

WCP-32

\section{Related to Pattern:}

Structured discriminator (1-of-m andjoin)

Control Discriminator WCP-9

(also known as: 1-out-of-M join.)

Structured partial join (n-of-m and-join) Control Discriminator WCP-30

Static partial join for multiple instances

(n:m \&-join)

Control Multiple_instance WCP-34

Multiple instances with a priori run-time knowledge (m:m and-join) 
B.5 Routing:

B.5.1 Data-based routing

Multiple instances with a priori design-

time knowledge ( $\mathrm{m}: \mathrm{m}$ and-join)

Control Multiple_instance

WCP-13

Multiple instances without a priori run-

time knowledge

Control Multiple_instance

WCP-15

Dynamic partial join for multiple

instances

Control Multiple_instance

WCP-36

Blocking discriminator (1-of-m and-join) Control

Discriminator

WCP-28

Blocking partial join (n-of-m and-join)

Control

Discriminator

WCP-31

Cancelling partial join for multiple

instances (n:m \&-join)

Control Multiple_instance

WCP-35

\section{B.5 Routing:}

\section{B.5.1 Data-based routing Data Routing WDP-40}

When the evaluation of data-based expressions have the ability to alter the controlflow within a case.

\section{Consider Issue:}

No valid choice

Data Robustness WCP-4

If there are multiple options available, then at least one outgoing branch needs to be valid. If this is not the case, then there is the potential for the process to hang.

\section{Consider Solution:}

Default choice

$$
\text { Control Robustness WCP-4 }
$$

\section{Related to Pattern:}

Exclusive choice (xor-split)

Control Split

WCP-4

(also known as: XOR-split, exclusive OR-split, conditional routing, switch, decision, case statement.)

Data-based routing

Data Routing

WDP -40 
B.6 State:

B.6.1 Using the token design pattern

Multi-choice (or-split)

Control Split

WCP-6

(also known as: Conditional routing, selection, OR-split, multiple choice.)

Deferred choice (time-based xor-split) Control State $\quad$ WCP-16

(also known as: External choice, implicit choice, deferred XOR-split.)

$\begin{array}{llll}\text { More than one exclusive choices } & \text { Control } & \text { Split } & \text { WCP-4 }\end{array}$

If there are multiple valid options available what do you do if you can only choose one.

\section{Consider Solution:}

Restricted choice

Control Split

WCP-4

Multi-choice

Control Split

WCP-4

\section{Related to Pattern:}

Exclusive choice (xor-split)

Control Split

WCP-4

(also known as: XOR-split, exclusive OR-split, conditional routing, switch, decision, case statement.)

Data-based routing

Data Routing

WDP-40

Multi-choice (or-split)

Control Split

WCP-6

(also known as: Conditional routing, selection, OR-split, multiple choice.)

Deferred choice (time-based xor-split) Control State

WCP-16

(also known as: External choice, implicit choice, deferred XOR-split.)

\section{B.6 State:}

\section{B.6.1 Using the token design pattern Data State CBP-4.2}

A token is an exclusively held resource that is analogous to a physical object. Only one party can hold a token at any given time and if the token is destroyed, it cannot be used at all. This pattern is crucial to how many modeling languages work (e.g. 
B.6 State:

B.6.1 Using the token design pattern

Name

Categ. Section

Source

BPMN), however the pattern can be used within a Business Processes as well, for example: in formal meetings only the person who "has the floor" is supposed to speak.

\section{Consider Issue:}

Missing tokens

Control Robustness

WCP-30

Individual abended threads/tokens would prevent a Join from resetting and failure to receive input on all of the incoming branches may result in the process instance (and possibly other process instances) stalling. Missing tokens could be a consequence of: - a design error - or that one of the tasks in the branch failing to complete successfully (e.g. as a consequence of it experiencing some form of exception) - or because the thread of control is passed outside of the branch. - or there are preceding splits or joins or they are un-structured in form (i.e. un-balanced splits and joins) (from WCP3)

\section{Consider Solution:}

Cancel region

Control Cancellation

WCP-29

Cancelling partial join (n-of-m and-join) Control

Robustness

WCP-30

Related to Pattern:

Structured partial join (n-of-m and-join) Control

Discriminator

WCP-30

Structured discriminator (1-of-m andjoin)

Control Discriminator

WCP-9

(also known as: 1-out-of-M join.)

Static partial join for multiple instances (n:m \&-join)

Multiple instances with a priori run-time knowledge (m:m \&-join)

Multiple instances with a priori designtime knowledge (m:m \&-join) 
B.6 State:

B.6.1 Using the token design pattern

Multiple instances without a priori run-

time knowledge

Control Multiple_instance WCP-15

Dynamic partial join for multiple

instances

Control Multiple_instance

WCP-36

Blocking partial join (n-of-m and-join)

Control Discriminator

WCP-31

Blocking discriminator (1-of-m and-join) Control

Discriminator

WCP-28

Cancelling partial join for multiple instances ( $\mathrm{n}: \mathrm{m} \&$-join)

A thread of control never arrives (lost tokens)

Control Robustness

WCP-3

The use of the Synchronization pattern can potentially give rise to a deadlock in the situation where one of the incoming branches fails to deliver a thread of control to the join construct.

\section{Consider Solution:}

Structured process (no

interrupts/exceptions)

$$
\text { Control Usage }
$$

WCP-3

Cancel region

Control Cancellation

WCP-29

\section{Related to Pattern:}

Synchronization (m-of-m and-join) Control Synchronizor $\quad$ WCP-3

(also known as: AND-join, rendezvous, synchronizer)

Thread merge (and-join) Control Discriminator WCP-41

Structured synchronizing merge (or-join) Control Synchronizor $\quad$ WCP-7

(also known as: Synchronizing join, synchronizer, OR-join)

Generalized and-join (m-of-m and-join) Control Synchronizor WCP-33

Local synchronizing merge (or-join) Control Synchronizor $\quad$ WCP-37

Detecting undefined vs empty/null Data Robustness WDP-34

Being able to differentiate between data elements that have an undefined 
B.6 State:

B.6.1 Using the token design pattern

Name

Categ. Section

Source

value and those that are merely empty or null.

Consider Solution:

Deciding undefined vs empty/null

Data Visibility

WDP-34

Related to Pattern:

Task precondition - data existence

Data Routing

WDP-34

Task postcondition - data existence

Data Routing

WDP-36

Task postcondition - data value

Data Routing

WDP-37

Task precondition - data value

Data

Routing

WDP-35

Related to Smell:

Existence check

Data Robustness

WDP-34

Bypassing synchronizing gateways

Data Internal

PAP-5.6

Dataflow bypassing synchronization gateways of alternative branches can cause deadlocks because data needed by a future operation may not be created along some branch Note: Bypassing forks and joins in parallel flows does not cause a deadlock, because all branches execute in parallel and thus, all business items always arrive through the connections i.e., all tasks receive their inputs as specified.

\section{Consider Solution:}

Make input optional

Data Transfer

PAP-5.7

Related to Smell:

Separate data and control flow channels

Data

Internal

WDP-9

Bypassing gateways

Data Internal

PAP-5.6

\section{Related to Solution:}

Explicit data passing via data channels

Data Internal

WDP-10

Discriminator missing tokens

Control Robustness

WCP-9

Individual abended threads/tokens would prevent a Discriminator from 
B.6 State:

B.6.1 Using the token design pattern

resetting and failure to receive input on all of the incoming branches may result in the process instance (and possibly other process instances) stalling.

\section{Consider Pattern:}

Cancelling discriminator (1-of-m and-

join)

Control Discriminator

WCP-29

Cancelling partial join (n-of-m and-join) Control

Discriminator

WCP-32

\section{Related to Pattern:}

Structured discriminator (1-of-m and-

Control Discriminator

WCP-9

(also known as: 1-out-of-M join.)

Structured partial join (n-of-m and-join) Control

Discriminator

WCP-30

Static partial join for multiple instances

(n:m and-join)

Control Multiple_instance

WCP-34

Multiple instances with a priori run-time knowledge (m:m and-join)

Multiple instances with a priori designtime knowledge (m:m and-join)

Multiple instances without a priori runtime knowledge

Control Multiple_instance

WCP-15

Dynamic partial join for multiple instances

Blocking discriminator (1-of-m and-join) Control

Discriminator

WCP-28

Blocking partial join (n-of-m and-join)

Control

Discriminator

WCP-31

Cancelling partial join for multiple instances ( $\mathrm{n}: \mathrm{m} \&$-join)

\section{Leaving tokens behind}

Control Cancellation

WCP-20

If cancellation of a case is enabled, it is assumed that precisely one of the cancelling transitions will fire cancelling all necessary enabled tasks. To 
B.6 State:

B.6.1 Using the token design pattern

achieve this, it is necessary that none of the cancelling transitions represent a state that is a superset of another possible state, otherwise tokens may be left behind after the cancellation. For example: During a mortgage application, the purchaser decides not to continue with a house purchase and withdraws the application. Someone needs to inform the property appraiser not to do an appraisal.

\section{Consider Solution:}

Quick kill

$\begin{array}{lll}\text { Control Cancellation } & \text { WCP-20- } \\ \text { Control Cancellation } & \text { WCP-20- }\end{array}$

\section{Related to Pattern:}

Cancel case

Control Cancellation WCP-20

(also known as: Withdraw case.)

Cancel multiple instance activity

Control Cancellation WCP-26

Cancel region

Control Cancellation WCP-25

Impact to interdependent activities

Resource Robustness

WRP-34

Cancelling tokens from outside region

Control Usage

WCP-29

Related to Pattern:

Cancel region

Control Cancellation

WCP-25

Impact to interdependent activities

Resource Robustness

WRP-34

Unfinished business

Control Termination

tbd

If the environment is not returned to the way it was found, this may cause problems for future activities and the cause of the problems may be hard to determine. For example: did the process reserve resources managed by other processes that need to informed they are no longer needed, etc. 
B.6.1 Using the token design pattern

\section{Related to Pattern:}

Cancel task

(also known as: Withdraw task.)

Explicit termination

Control Termination

WCP-43

Not deleted on time

Data Performance

DAP-9

If large amounts of data are being processed, then unnecessary storage may be tied up if the data is not deleted immediately after it is no longer needed, even if it is meaningless with out its original context, or it will be automatically cleaned up later (for example when the software program exits)

\section{Related to Smell:}

Not cleaning up data

$\begin{array}{lll}\text { Data } & \text { Termination } & \text { DAP-7 } \\ \text { Data } & \text { Termination } & \text { DAP-7 }\end{array}$

Never destroyed

Termination

DAP-7

If there is a scenario in which data is created but not destroyed afterwards, it may continue to exist and use up resources after the workflow case completes. If the data is sensitive this could become a security or privacy issue.

\section{Related to Smell:}

Not cleaning up data

$\begin{array}{lll}\text { Data } & \text { Termination } & \text { DAP-7 } \\ \text { Data } & \text { Robustness } & \text { tbd }\end{array}$

Token backlog

l) waiting to be processed - for whatever reason. If there isn't a way to keep track of them all, some may be lost causing additional problems to tasks that needed them to complete. (From Börger et al. 2008)

\section{Related to Solution:}

Wait to be processed

Control Trigger

WCP3 
B.6 State:

B.6.1 Using the token design pattern

\begin{tabular}{rlll} 
Name & Categ. & Section & Source \\
\hline Wait for data & Control & Trigger & WDP-9 \\
Data overload & Data & Internal & WDP-11
\end{tabular}

Consider Solution:

Allow block to create more data elements to handle overload

Data Internal $\quad$ WDP-11

Bind sub-workflow data to block task

data

Data Internal

WDP-11

\section{Related to Pattern:}

Sub-workflow decomposition to block task

Data Internal WDP-11

Block task to sub-workflow

decomposition

Data Internal

WDP-10

\section{Related to Solution:}

Merge changes strategy

Data Transfer

WDP-29

Transfer is not atomic.

Data Transfer

CBP-4.2

If control of something depends having possession of an object (for example a baton, or a crown) but the object is only exists digitally (e.g. a token), then it is possible for that object to exist in more than one place or cease to exist completely. For example, when transferring money electronically, the value must be deleted from one account and added to another. During this time, or if something went wrong, then the money could be in two accounts or no longer existing at all.

\section{Consider Solution:}

Clean-up after transfer

Data Transfer

CBP-4.2

\section{Related to Pattern:}

The token design pattern

Data State

CBP-4.2 
B.6 State:

B.6.1 Using the token design pattern

\begin{tabular}{llll} 
Name & Categ. & Section & Source \\
\hline Object assumed to be protected & Control & Concurrency & CBP-2.2
\end{tabular}

(also known as: Code assumed to be protected)

Assuming access by others is prevented when it actually isn't (like people who assume a bathroom door is always locked when the bathroom is in use)

\section{Related to Smell:}

Using a lock when making changes to something shared

$\begin{array}{lll}\text { Data } & \text { Concurrency } & \text { CBP-2.2 } \\ \text { Control } & \text { Concurrency } & 2.2 .3\end{array}$

\section{Wrong lock or no lock}

If access to something is only controlled by an agreed upon "protocol" that has no way to absolutely control that access, then problems may occur when the protocol is not followed correctly or at all. For example people who are used to locked bathroom doors showing occupancy vs people who expect others to knock before opening the door.

\section{Non-protected version is used instead of} protected version

$$
\text { Control Usage }
$$

CBP-4.1

If not all the ways to access something are protected then using the unprotected access (unnecessarily) may cause problems. For example always accessing a computer through an administrator (or root) account can cause a lot of problems.

\section{Related to Pattern:}

Protection and subclassing Control Concurrency CBP-4.1

(also known as: Confinement and Subclassing)

\section{Double-checked locking \\ Data Creation \\ CBP- \\ 2.2 .4}

This comes from software, where a newly created object is available for others to use before it has been completely initialized. Similarly a report could be released for others to use while the author is still gathering information. 
B.7 Termination:

B.7.1 Multiple termination points

Name

Categ. Section

Source

CBP-

Nonatomic operations assumed to be atomic Control Concurrency

2.2.1

Sometimes an activity that looks like it cannot be interrupted (self contained, i.e. atomic) can be because of the underlying activities which implement it. For example asking one person to sign a report not realizing they are really the contact person into an organization with its own approval process.

\section{Consider Pattern:}

Critical section

$\begin{array}{lll}\text { Control } & \text { State } & \text { WCP-39 } \\ \text { Control } & \text { State } & \text { WCP-40 } \\ \text { Data } & \text { Transfer } & \text { WDP-31 } \\ & & \text { CBP- } \\ \text { Control } & \text { Concurrency } & 2.2 .2\end{array}$

Two-stage access bug pattern

CBP-

Sometimes a sequence of operations needs to be protected but instead it was wrongly assumed that protecting each operation separately is enough. For example transferring money electronically from one bank to another. It is not desirable if at any time that person appeared to have twice the amount of money or none.

\section{B.7 Termination:}

\section{B.7.1 Multiple termination points Control Termination PAP-7.1}

(also known as: Stop Node in Parallel Execution Branches)

In some modeling languages there are stop nodes which shutdown the whole process as well as end nodes which just terminate an execution branch. The stop nodes may also be needed to release data from a sub-process to its parent process when it finishes. Therefore a model might contain stop/shutdown nodes on multiple branches. 
B.7 Termination:

B.7.2 Not cleaning up data

Name

Categ. Section

Source

Consider Issue:

Not all control paths may be ready to terminate

Control Termination

PAP-7.1

Stop nodes ending parallel branches always terminate the whole process, even if tasks have not finished their execution, which may leave shared data in an incomplete (i.e. corrupted) state.

\section{Consider Pattern:}

Implicit termination

Control Termination

WCP-11

Consider Solution:

Create single termination point.

$\begin{array}{lll}\text { Control } & \text { Termination } & \text { WCP-43 } \\ \text { Control } & \text { Termination } & \text { PAP-7.1 }\end{array}$

Use a single end or stop node,

Control Termination

WCP-43

Explicit termination

Control Termination

DAP-7

\section{B.7.2 Not cleaning up data}

Data Termination

Data is not cleaned up after use, or ever

\section{Consider Issue:}

Never destroyed

Data Termination

DAP-7

If there is a scenario in which data is created but not destroyed afterwards, it may continue to exist and use up resources after the workflow case completes. If the data is sensitive this could become a security or privacy issue.

\section{Not deleted on time Data Performance DAP-9}

If large amounts of data are being processed, then unnecessary storage may be tied up if the data is not deleted immediately after it is no longer needed, even if it is meaningless with out its original context, or it will be automatically cleaned up later (for example when the software program 
B.7 Termination:

B.7.2 Not cleaning up data

\begin{tabular}{cccc} 
Name & Categ. & Section & Source \\
\hline exits) & & & \\
Unfinished business & Control & Termination & tbd
\end{tabular}

If the environment is not returned to the way it was found, this may cause problems for future activities and the cause of the problems may be hard to determine. For example: did the process reserve resources managed by other processes that need to informed they are no longer needed, etc.

\section{Related to Pattern:}

Cancel task

Control Cancellation WCP-19

(also known as: Withdraw task.)

Explicit termination Control Termination WCP-43

Leaving tokens behind Control Cancellation WCP-20

If cancellation of a case is enabled, it is assumed that precisely one of the cancelling transitions will fire cancelling all necessary enabled tasks. To achieve this, it is necessary that none of the cancelling transitions represent a state that is a superset of another possible state, otherwise tokens may be left behind after the cancellation. For example: During a mortgage application, the purchaser decides not to continue with a house purchase and withdraws the application. Someone needs to inform the property appraiser not to do an appraisal.

\section{Consider Solution:}

Quick kill

$$
\begin{array}{lll}
\text { Control } & \text { Cancellation } & \text { WCP-20- } \\
\text { Control Cancellation } & \text { WCP-20- }
\end{array}
$$$$
\text { By-pass }
$$

\section{Related to Pattern:}

Cancel case

Control Cancellation WCP-20

(also known as: Withdraw case.)

Cancel multiple instance activity

Control Cancellation

WCP-26

Cancel region

Control Cancellation

WCP-25 
B.8 Transfer:

B.8.1 Passing data to a different data type

\begin{tabular}{llll} 
Name & Categ. & Section & Source \\
\hline $\begin{array}{l}\text { Impact to interdependent activities } \\
\text { Related to Smell: }\end{array}$ & Resource & Robustness & WRP-34 \\
Using the token design pattern & Data & State & CBP-4.2 \\
Cancelling tokens from outside region & Control & Usage & WCP-29 \\
Related to Pattern: & & & \\
Cancel region & Control & Cancellation & WCP-25 \\
Impact to interdependent activities & Resource & Robustness & WRP-34
\end{tabular}

\section{B.8 Transfer:}

\section{B.8.1 Passing data to a different data type}

Data Transfer

WDP-32

The Sender and Receiver use different data types when passing information

\section{Consider Issue:}

Different input data types Data Transfer WDP-32

When applying a transformation function to a data element prior to it being passed to another process component, the transformation function has to have access to the same data elements as the receiving process component. It is also possible for tranlation errors to occur.

\section{Consider Pattern:}

Transformation - input

Data Transfer

WDP-32

Transformation - output

Data Transfer

WDP-33

\section{Related to Pattern:}

Transfer by value - incoming

Data Transfer

WDP-27

Transfer - copy in/copy out

Data Transfer

WDP-29

Transfer by value - outgoing

Data Transfer

WDP-28 
B.9 Trigger:

B.9.1 Events as control flow

\begin{tabular}{|c|c|c|c|}
\hline Name & Categ. & Section & Source \\
\hline Different output data types & Data & Transfer & WDP-33 \\
\hline \multicolumn{4}{|c|}{$\begin{array}{l}\text { When applying a transformation function to a data element immediately } \\
\text { prior to it being passed out of a process component, the transformation } \\
\text { function has access to the same data elements as the process component that } \\
\text { initiates it. It is also possible for translation errors to occur. }\end{array}$} \\
\hline \multicolumn{4}{|l|}{ Consider Pattern: } \\
\hline Transformation - output & Data & Transfer & WDP-33 \\
\hline Transformation - input & Data & Transfer & WDP-32 \\
\hline \multicolumn{4}{|l|}{ Related to Pattern: } \\
\hline Transfer by value - outgoing & Data & Transfer & WDP-28 \\
\hline Transfer - copy in/copy out & Data & Transfer & WDP-29 \\
\hline Transfer by value - incoming & Data & Transfer & WDP-27 \\
\hline
\end{tabular}

\section{B.9 Trigger:}

\section{B.9.1 Events as control flow Data $\quad$ Trigger $\quad$ PAP-6.1}

Frequently control flow is used to capture events.

\section{Consider Issue:}

Control flow does not carry information as events usually do

However, not all modeling languages' control flow mechanisms carry any information as events usually do. Consequently, a model that captures events with control flow can lead to a variety of problems (depending on the language and how the event was modeled).

\section{Consider Solution:}

Use triggers or events 
B.10 Usability:

B.10.1 Multiple connections between activities

Name

Categ. Section

Source

\section{B.10 Usability:}

\section{B.10.1 Multiple connections between activities}

Data Usability

PAP-5.3

Complex control and data flows easily lead to multiple connections in process models, which can result in cluttered models.

Consider Issue:

Complicated models are hard to maintain and can hide errors

Data Usability

PMG

Consider Solution:

Use dangling inputs and outputs to reduce clutter.

Data Usability

PAP-5.2

Use as few elements in the model as possible

Decompose a model with more than 50 elements

Control Usability

PMG-7

Minimize the routing paths per element

Control Usability

PMG-2

Reduce mixing data and control flow in a model.

Data Usability

PAP-5.3

Use one start and one end event

Control Usability

PMG-3

Model as structured as possible

Control Usability

PMG-4

Avoid or routing elements

Control Usability

PMG-5

Use verb-object activity labels

Control Usability

PMG-6

\section{Related to Smell:}

Cluttered data-flow models

Data Usability

PAP-5.2 
B.11 Usage:

B.10.2 Cluttered data-flow models

Name Categ.

Section Source

Multiple connections between activities can result in errors Data Usability

PAP-5.3

Multiple connections, for short multi-connections all start in the same activity or gateway and all end in one other activity or gateway. These connections lead to unnecessary redundancy if the multi-connections only involve control flow. If the multi-connections are associated with the same business item, they can easily lead to modeling errors.. Data multiconnections usually point to a modeling problem where users either tried to pass the same item to several activities or intended to express that two different instances of the item are passed.

\section{Consider Solution:}

Reduce mixing data and control flow in a model.

Data Usability

PAP-5.3

Minimize the routing paths per element Control

Usability

PMG-2

\section{B.10.2 Cluttered data-flow models}

Data Usability

PAP-5.2

Consider Issue:

Complicated models are hard to maintain and can hide errors Data Usability PMG

PLEASE SEE earlier description

\section{B.11 Usage:}

\section{B.11.1 Data is written to, but never used Data Usage DAP-2}

\section{Consider Issue:}

Strongly redundant data Data Usage DAP-2

A data element is strongly redundant if there is a writing activity after which in all possible continuations of the execution is completed. Strongly redundant data indicates in most situations a real flaw in the workflow. 
B.11 Usage:

B.11.2 Data is over-written without being used first

\begin{tabular}{lccc} 
Name & Categ. & Section & Source \\
\hline Dangling output means lost data & Data & Internal & PAP-5.1
\end{tabular}

Dangling data outputs show that a task or subprocess produced some data but this data is not used subsequently in the process.

\section{Related to Smell:}

Dangling inputs and outputs

Data Internal

PAP-5.1

\section{Related to Solution:}

Use dangling inputs and outputs to reduce clutter.

$$
\text { Data Usability }
$$

PAP-5.2

Weakly redundant data

Data Usage

DAP-3

A data element is weakly redundant if there is some execution scenario in which it is written but never read afterwards, i.e. before it is destroyed or the total case's execution is completed. Weakly redundant data is not necessarily a flaw. For example, to improve performance some data may be computed in advance, even though it may not be needed later.

\section{B.11.2 Data is over-written without being used first}

Data Usage

DAP-4

\section{Consider Issue:}

Strongly lost data

Data Usage

DAP-4

A data element is strongly lost if there is a writing activity after which in all possible execution paths this element gets overwritten without having been read first. This result in lost resources if the data element is a reference to other data, or it may only be a performance problem.

\section{Weakly lost data}

Data Usage

DAP-5

A data element is weakly lost if there is an execution sequence in which it is overwritten without been read first. This may not be a flaw, for example capturing data in advance which may be updated at any time before it is actually used. 
B.12 Visibility:

B.11.3 Data is read or destroyed before being initialized or written to.

\section{B.11.3 Data is read or destroyed before being initialized or written to.}

$$
\text { Data Usage }
$$

DAP-1

\section{Consider Issue:}

Missing data

$$
\text { Data Usage }
$$

DAP-1

The situation where some data element needs to be accessed, i.e. read or destroyed, but either it has never been created or it has been deleted without having been created again.

Twice destroyed

Data Termination

DAP-8

A data element is twice destroyed if there is a scenario in which it is destroyed twice in a row without having been created in between.

\section{B.12 Visibility:}

\section{B.12.1 Reusing names for data \\ Data Visibility \\ WDP-1}

Reusing names for data elements in different parts of the process model.

\section{Consider Issue:}

Global name clash

Data Visibility

WDP-1

Name clash can occur when a task names a data element the same as in another part of the model which can be access from within that task. This may cause an immediate error or data may be unintentionally over written or hidden from view without warning.

\section{Consider Solution:}

Task data scope

Data Visibility

WDP-1

Related to Pattern:

Task data

Data Visibility

WDP-1 
B. 12 Visibility:

B.12.1 Reusing names for data

\section{Related to Solution:}

Implicit case data access

$\begin{array}{lll}\text { Data } & \text { Visibility } & \text { WDP-5 } \\ \text { Data } & \text { Visibility } & \text { WDP-2 }\end{array}$

Cascading name clash

Visibility

If sub-processes can contain sub-processes, etc., then if data elements are accessible by sub-process, are they likewise accessible by their sub-processes, potentially increasing the chances of clashes.

\section{Consider Solution:}

Task data scope

WDP-1

Uniquely name concurrent non-

independent data

Data Visibility

WDP-10

\section{Related to Pattern:}

Block data

$\begin{array}{lll}\text { Data } & \text { Visibility } & \text { WDP-2 } \\ \text { Data } & \text { Visibility } & \text { WDP-6 }\end{array}$

Folder data name clash

Control Concurrency

WDP-6

Access only one folder at a time

Related to Pattern:

Folder data

$\begin{array}{lll}\text { Data } & \text { Visibility } & \text { WDP-6 } \\ \text { Data } & \text { Visibility } & \text { WDP-3 }\end{array}$

Scope data

Data Visibility

WDP-3

Data elements in the block and subprocess not independent

Data Internal WDP-10

if data elements are accessible from both a task and its sub-processes, then the potential exists for concurrency issues to arise as tasks executing in parallel update the same data element.

\section{Consider Solution:}

Uniquely name concurrent nonindependent data

Data Visibility

WDP-10 
B. 12 Visibility:

B.12.1 Reusing names for data

Name

Categ.

Section

Source

Related to Solution:

Explicit data passing via parameters

Data Internal WDP-10

Scope data name clash

Data Visibility

WDP-3

Consider Solution:

Task data scope

Data Visibility

WDP-1

Related to Pattern:

Scope data

Data Visibility WDP-3

Folder data

Data Visibility

WDP-6

Subprocesses can access everything

Data Visibility

WDP-10

Information is accessible by everything as soon as it is available, so data is passed to other tasks simply by being made available, hence concurrent tasks may try to make changes to the same data at unexpected times.

\section{Consider Pattern:}

Block data

Data Visibility

WDP-2

\section{Related to Smell:}

Concurrency management

Data Concurrency

WDP-29

Related to Solution:

Implicit data passing

Data Internal WDP-10

